\title{
Frequency-dependent feedback constrains plant community coexistence
}

Maarten B. Eppinga ${ }^{1 *}$, Mara Baudena ${ }^{1}$, Daniel J. Johnson ${ }^{2}$, Jiang Jiang ${ }^{3}$, Keenan M.L. Mack $^{4}$, Allan E. Strand ${ }^{5}$ James D. Bever ${ }^{6}$

${ }^{1}$ Copernicus Institute of Sustainable Development, Environmental Sciences, Faculty of Geosciences, Utrecht University, Heidelberglaan 2, P.O. Box 65, 3508 TC Utrecht, The Netherlands

${ }^{2}$ Department of Earth and Environmental Sciences, Los Alamos National Laboratory, Los Alamos, NM, United States

${ }^{3}$ Department of Soil and Water Conservation, Nanjing Forestry University, Nanjing, Jiangsu, China

${ }^{4}$ Department of Biology, Illinois College, Jacksonville, IL, United States

${ }^{5}$ Department of Biology, Grice Marine Laboratory, College of Charleston, Charleston, SC, United States

${ }^{6}$ Department of Ecology and Evolutionary Biology and Kansas Biological Survey, University of Kansas, Lawrence, KS, United States

* Corresponding author: M.B.Eppinga@uu.nl 


\begin{abstract}
Ecological theory suggests that coexistence of many species within communities requires negative frequency-dependent feedbacks to prevent exclusion of the least fit species. For plant communities, empirical evidence of negative frequency-dependence driving species coexistence and diversity patterns is rapidly accumulating, but connecting these findings to theory has been difficult as corresponding theoretical frameworks only consider small numbers of species. Here, we show how frequency-dependent feedback constrains community coexistence, regardless of the number of species and inherent fitness inequalities between them. Any interaction network can be characterized by a single community interaction coefficient, $I_{C}$, which determines whether community-level feedback is positive or negative. Negative feedback is a necessary (but not sufficient) condition for persistence of the entire community. Even in cases where the coexistence equilibrium state cannot recover from perturbations, $I_{C}<0$ can enable species persistence via cyclic succession. The number coexisting species is predicted to increase with the average strength of negative feedback. This prediction is supported by patterns of tree species diversity in more than 200,000 deciduous forest plots in the eastern United States, which can be reproduced in simulations that span the observed range of community feedback. By providing a quantitative metric for the strength of negative feedback needed for coexistence, we can now integrate theory and empirical data to test whether observed feedback-diversity correlations are strong enough to infer causality.
\end{abstract}




\section{Introduction}

Despite decades of research, it remains surprisingly challenging to explain how species competing for the same resources can coexist in the large numbers observed in plant communities $^{1-7}$. Ecological theory states that stabilizing mechanisms creating negative frequency-dependent feedbacks are needed to prevent competitive exclusion due to species differences in fitness ${ }^{6-8}$. Recent empirical studies of species-rich plant communities are in qualitative agreement with the expected connection between negative frequency-dependent feedback and patterns of species abundance ${ }^{9,10}$ and species richness ${ }^{11-13}$. Until now, utilization of these data for more direct tests of ecological theory is hampered by the lack of a theoretical analytical framework linking species interactions to community diversity and stability. In contrast to studies of evolutionary game dynamics ${ }^{14}$, theoretical ecological studies have focused either on coexistence of small numbers of species ${ }^{3}$, or on sufficiently large communities enabling a distributional approach based on probabilities (of particular species interactions ${ }^{15}$ ). Alternatively, coexistence has been studied for equivalent species ${ }^{16}$, species exerting equal effects on all competitors ${ }^{17}$, (anti-)symmetric species interactions ${ }^{5,7}$ or for species experiencing strictly hierarchical competition ${ }^{2}$. These assumptions enable a detailed description of potential plant community dynamics, but do not consider asymmetric competitive interactions. In addition, the above approaches do not represent the forces driving negative frequency-dependent feedbacks at the community level.

Focusing on a specific mechanism through which frequency-dependent feedbacks may arise from asymmetric species interactions, plant-soil feedback theory has been developed by means of models that can be directly parameterized with data from pot or field experiments, providing a promising means to integrate experiments with theory ${ }^{18-20}$. Yet, theoretical development and empirical testing of plant-soil feedback theory has been limited to small numbers of interacting species ${ }^{18-23}$. Here, we generalize the plant-soil feedback model ${ }^{18}$ (see 
Supplementary Information section 1), yielding a generic phenomenological model of frequency-dependent interactions. When rewriting the species' density dynamics into frequency dynamics, this generalized model projects onto the well-known replicator equation originating from evolutionary game theory ${ }^{14}$. The replicator equation can be solved analytically for communities containing any number of species. This procedure does not require an assumption of species equivalence, meaning that equilibrium solutions retain species-specific parameters describing the growth for each species. We also derive a fundamental criterion for species persistence. Our results provide a framework to assess the importance of empirically observed frequency-dependent interactions in maintaining coexistence of highly diverse plant communities (Fig. 1).

\section{Results \& Discussion}

The proposed framework consists of a set of ordinary differential equations describing plant frequencies and hence community dynamics over time (Supplementary Information section 1). The only parameters in this model are the coefficients $\sigma_{i j}$, which quantify the growth rate of species $i$ in an environment dominated by species $j$ (Fig. 1). Thus, when these coefficients are set to non-neutral values, species fitness becomes frequency-dependent, creating a feedback between community composition and the growth of each species. For a system of two species, net feedback is described by the two-species interaction coefficient $I_{S}{ }^{18,19}$ :

$$
I_{s}=\sigma_{11}-\sigma_{12}-\sigma_{21}+\sigma_{22}
$$

Provided that the coexistence equilibrium is feasible (i.e. both plant species have a positive frequency at equilibrium), $I_{S}<0$ will generate negative feedback, and the equilibrium is stable. Alternatively, $I_{S}>0$ will generate positive feedback and the equilibrium is unstable, meaning that one of the plant species will be excluded ${ }^{18,19}$ (Supplementary Information section 2). $I_{S}$ can thus be interpreted as a pair-wise feedback: the sum of two species-specific 
feedbacks, which are described by the difference between a species' conspecific and its heterospecific responses (thus, the species-specific feedback of plant species 1 , for example, is given by $\left.{ }^{24}: \sigma_{11}-\sigma_{21}\right)$. For the two-species case, the equilibrium frequency for each plant species is then given by the ratio between its competitor's species-specific feedback and the pair-wise feedback ${ }^{18}$, yielding:

$\hat{P}_{1}=\frac{\sigma_{22}-\sigma_{12}}{I_{S}}, \hat{P}_{2}=\frac{\sigma_{11}-\sigma_{21}}{I_{S}}$

in which $\hat{P}_{i}$ indicate the species' equilibrium frequencies. These results can be generalized from two to any number of species (Supplementary Information section 3). General results can be obtained using the interaction matrix:

$$
\mathbf{A}=\left[\begin{array}{cccc}
\sigma_{11} & \sigma_{12} & . . & \sigma_{1 n} \\
\sigma_{21} & . . & . . & . . \\
. . & . . & . . & . . \\
\sigma_{n 1} & . . & . . & \sigma_{n n}
\end{array}\right]
$$

As in the two species case, the frequency of a given species at the coexistence equilibrium is the ratio between the feedback effects that this species experiences from all competitors, and the sum of all feedback effects within the community:

$$
\hat{P}_{i}=\frac{\operatorname{det} \mathbf{A}_{i}}{\sum_{j=1}^{n} \operatorname{det} \mathbf{A}_{j}}
$$

where $\mathbf{A}_{i}$ indicates the interaction matrix $\mathbf{A}$, but with the $i^{\text {th }}$ column being replaced with a column vector of ones (Fig. 1). Thus, based on the interaction structure within the plant community (equation [3]), the equilibrium frequencies for species at coexistence can be calculated (equation [4]). Stability of this equilibrium can then be assessed by standard eigenvalue analysis of the system's Jacobian matrix. We used this approach to study community assembly and the resulting plant species richness (quantified as the maximum number of species that were found to stably coexist) under various regimes of negative 
feedback (Fig 2; see also Methods; Supplementary Information section 6). We found that under more negative feedback, indicated by the system's average pair-wise feedback ${ }^{10,19,23}$, communities with higher species richness could be assembled (Fig. 2). For a plant community in which the number of species was fixed, more negative feedback increased the resilience of the coexistence equilibrium (Fig. 3a, in which resilience is measured as the return time after perturbation $\left.{ }^{25}\right)$. In addition, more negative feedback increased community evenness (Fig. 3b). The fact that average pair-wise feedback correlates well with these community level properties is surprising, because pair-wise descriptors provide no formal (i.e. mathematically formulated) conditions for coexistence in communities of more than two species ${ }^{7}$. Therefore, to establish whether pair-wise descriptors can be used as an indicator of community-level feedback, a formal measure of the latter is also needed.

We found that for the model framework analysed, the two-species interaction coefficient $I_{S}$ can be generalized to the community level, yielding an exact measure for community-level feedback. More specifically, the generalized community interaction coefficient, $I_{C}$, is given by (Supplementary Information section 3):

$$
I_{C}=(-1)^{n} \sum_{j=1}^{n} \operatorname{det} \mathbf{A}_{j}
$$

A pattern generally observed in empirical studies is that conspecific effects (and variation therein) are relatively strong compared to heterospecific effects (and variation therein) $)^{11-13}$. Under these conditions, our measure of community-level feedback correlated well with the average strength of pair-wise feedback (equation [1]; Fig. 3c; Supplementary Information section 6). This result did not only hold for asymmetric interaction matrices (Fig. 3c), but also for (anti)-symmetric interaction matrices (Supplementary Information section 3). This monotonic relation between average pair-wise feedback and community-level feedback thus provides an explanation for recent empirical observations of average pair-wise feedback correlating well with community diversity ${ }^{11-13}$. 
We could prove that negative community-level feedback, $I_{C}<0$, presents a necessary condition for persistence of all species (Supplementary Information section 3). Interestingly, the value of $I_{C}$ is not affected by inherent fitness inequalities ${ }^{6}$ between species (Supplementary Information section 7). Following the community assembly approach (see Methods), we found that negative feedback could still be present in communities for which the real part of the leading eigenvalue of the coexistence equilibrium was positive (Supplementary Information section 4). Thus, even when small perturbations will drive the community away from the coexistence equilibrium point, negative community-level feedback may still prevent species extinction. In this case, species abundances fluctuate, but dominance is passed on from one species to another in ways that create repeating loops (e.g. A $\rightarrow \mathrm{B} \rightarrow \mathrm{C}$ $\rightarrow$ A) referred to as cyclic succession ${ }^{22,26}$ (Supplementary Information section 4). However, $I_{C}<0$ is not a sufficient condition for persistence of all species within a community (Supplementary Information section 3). These observations highlight the importance of knowing the exact structure of the right-hand side of equation [3], as the loops creating the cyclic succession dynamics are not conserved when all the heterospecific effects that a species experiences have been averaged, for example (Supplementary Information section 4). Parameterizing the right-hand side of equation [3] requires full-factorial experimental designs, the size of which increase rapidly with increasing plant community size. However, even in cases where information is incomplete, our theoretical framework can be used to infer plant community characteristics, by constraining the right-hand side of equation [3] within a realistic range of parameter values (rather than providing the exact value of each parameter). Observational data as recently gathered in diverse forest ecosystems ${ }^{9,11-13}$ can be used for this aim. To illustrate this potential, we analysed data from North American deciduous forests east of the $100^{\text {th }}$ meridian, aggregating surveys from 207,444 plots to $2 \times 2$-degree grid cells ${ }^{11}$ (Fig. 4a). Previous measures of conspecific and averaged heterospecific frequency 
dependence $^{11}$ can be translated into the model parameters used in the current framework (equations [3,4]; Supplementary Information section 5). Focusing on grid cells containing sufficient data for five or more tree $\operatorname{species}^{11}$ (Supplementary Information section 5), we distinguished four community diversity classes (5-15 species, 15-25 species, 25-35 species and $\geq 35$ species occurring in a grid cell, Fig. 4a). We used the previously derived measures for conspecific and averaged heterospecific density dependence ${ }^{11}$ to calculate for each of these cells the average pair-wise feedback experienced by the tree species present. Consistent with previous observations of feedback-diversity correlations ${ }^{11,12}$, the average pair-wise feedback became more strongly negative with increasing community diversity (see the coloured bars in Fig. 4b). A hitherto unanswered question, however, is whether these observed differences in feedback are indeed strong enough to drive (rather than correlate with) the variation in community diversity. Therefore, we compared the pattern obtained from these observations with independently assembled theoretical communities that spanned the same range of diversity classes. In these theoretical communities, the differences in species diversity were entirely driven by variation in the strength of community-level feedback. The conspecific and heterospecific coefficients in these theoretical communities were varied over the same range as observed in the field data (Supplementary Information section 5). The community assembly process was carried out as before (Fig. 2; see Methods). We found that the variation in pair-wise feedback strength between these theoretical communities (the grey bars in Fig. 4b) was similar to the variation empirically observed (Fig. 4b). Although a correlation between feedback and diversity has been previously observed in northern American deciduous forests ${ }^{11,12}$, application of the framework developed here verifies that the observed variation in feedback is in theory strong enough to be a causal factor driving the observed variation in community diversity (Fig. 4). 
Our results further suggest that in the absence of inherent fitness differences between species, a species' experienced feedback will positively correlate with its relative abundance (equation [4]), as has been commonly observed in the field ${ }^{10,11,13,27}$. Although inherent fitness differences may decouple this link between experienced feedback and relative abundance, we found that such differences would not undermine the stabilizing force of community-level negative feedback (Supplementary Information section 7). Together, these results provide an explanation for recent observations of consistent signatures of negative feedback in tropical and temperate forest communities, but declining slopes of feedback-abundance relationships with increasing latitude ${ }^{13}$.

Quantitative studies of ecological networks have mainly focused on food webs and mutualistic networks ${ }^{15,28}$ or have focused on competition within a trophic level by studying networks that are symmetric (e.g. mimicking overlap in resource use ${ }^{7}$ ) or anti-symmetric (where there is a winner and a loser in each two-way interaction ${ }^{5}$, see also ${ }^{3}$ ). Frequencydependent feedback as studied here considers a community in which plants interact through a fully connected network but where interactions between species can be asymmetric (e.g. species A strongly affects species B, but B only weakly affects A). Empirical evidence for specific mechanisms creating this latter kind of interaction structure is rapidly increasing. Examples include density-dependent seedling mortality ${ }^{9,10}$, plant-soil feedbacks initiated by invertebrate fauna ${ }^{29}$, by soil pathogens ${ }^{21}$ or by mycorrhizal associations ${ }^{27}$ and auto-toxic effects of extra-cellular self-DNA originating from plant litter ${ }^{30}$. Understanding the contribution of these mechanisms to plant community coexistence will be contingent on moving beyond the study of interactions between small numbers of species ${ }^{18-20}$. The theoretical framework presented here provides a crucial link between empirical estimates of frequency-dependent feedback and its consequences for community stability and species diversity patterns. 


\section{Methods}

\section{Strength of negative frequency-dependent feedback and species richness of communities}

A random interaction matrix for 1,000 species was generated. From this matrix, communities were assembled, starting with two species randomly selected. Analytical calculation of the coexistence equilibrium (see equations $[3,4]$ in the main text) and the relevant eigenvalues of the corresponding Jacobian matrix (Supplementary Information section 3) were then calculated to assess whether this two species coexistence equilibrium was stable. If coexistence was feasible and stable, another species was randomly selected and added to the community, after which the procedure was repeated. This process continued until the community was no longer locally stable, or until a predetermined species number was reached, at which the community resilience (by means of the magnitude of the relevant eigenvalue with the maximal real part ${ }^{25}$ ) and evenness (using Pielou's index) were assessed. Average feedback strengths in the 1000-species matrix were varied by manipulating the strengths of conspecific effects, relative to the strengths of heterospecific effects. In the simulations, conspecific coefficients ranged between 0.05 and 0.3 , and heterospecific coefficients ranged between 0.3 and $0.9^{10,23}$. The subsets considered within these ranges varied along the feedback gradient (see Supplementary Information section 6 for details). We verified, however, that alternative parameterizations led to qualititatively similar feedbackcommunity diversity relations (Supplementary Information section 6).

\section{Plant-soil feedbacks and the Forest Inventory and Analysis dataset}

We analyzed tree species diversity of forest plots located east of the $100^{\text {th }}$ meridian, which are part of the US Forest Service's Forest Inventory and Analysis (FIA) dataset. These data were previously extracted and pre-processed ${ }^{11}$. In this previous work, the strength of density dependence was inferred from (maximum likelihood) fitting a negative exponential relation 
between observed seedling densities and tree density in the surrounding plot. Here, we used the dataset described in ref $^{31}$ to obtain exponential coefficients, using cumulative basal area as the predictor variable for adult density. The dataset analysed in ref. ${ }^{31}$ differed from ref. ${ }^{11}$ in that it also included ecologically relevant joint absences of seedlings and adult trees within forest plots, which tends to increase coefficient values ${ }^{32}$. The exponential coefficients were used to calculate values for the conspecific frequency-dependent effect parameters, $\sigma_{i i}$, and for the average heterospecific effect, $\sigma_{i j}$, on each species (Supplementary Information section 5). Theoretical results showed that average pair-wise feedback (equation [1] in the main text) correlates well with community-level feedback (see Fig. 3c of the main text and Supplementary Information sections 3 and 6). Average pair-wise feedback could also be calculated for the FIA data selection, as it only requires information of the average of heterospecific effects that a tree species experiences ${ }^{11}$ (Supplementary Information section 5). We calculated average pair-wise feedback strengths aggregating observations from different plots at the level of grid cells spanning 2 degrees by 2 degrees (latitude $\mathrm{x}$ longitude) ${ }^{11}$ (Supplementary Information section 5). In total, average pair-wise feedback was calculated for 113 grid cells in which the average pair-wise feedback could be calculated for five or more tree species (up to 51 tree species). These calculated feedbacks were compared to a null-prediction in which the associations between seedlings and adults within each grid cell were randomized (Supplementary Information section 5). Patterns of feedback strength and observed community diversity in the corresponding grid cells were then compared to patterns in theoretical communities in which community diversity was determined by the strength of community-level feedback. As the data provided row-averaged values of heterospecific effects (i.e. the off-diagonal entries of the community matrix; see equation [3] in the main text), the model parameters in simulations could be constrained, i.e. varied over the same 
range as observed in the data, including the ranges assumed for within-row and between-row variation in heterospecific effects (Supplementary Information section 5).

\section{Data Availability Statement}

The US Forest Service's Forest Inventory and Analysis data that support the findings of this study are available from www.fia.fs.fed.us/tools-data/, under FIA Data Mart. The database subset that was used in the current study is available from the corresponding author upon reasonable request.

\section{Code Availability Statement}

The Matlab scripts and R scripts written for this study only contain standard functions and analysis methods. Scripts are available from the corresponding author upon reasonable request.

\section{Acknowledgements}

This work was conducted as a part of the 'Plant-soil feedback theory' Working Group at the National Institute for Mathematical and Biological Synthesis (NIMBioS), sponsored by the National Science Foundation through NSF Award \#DBI-1300426, with additional support from The University of Tennessee, Knoxville. We thank K.C. Abbott, J. Bauer, L.S. Comita, K.M. Crawford, S.A. Mangan, J. Umbanhowar and K.N. Suding for discussions of this work during working group meetings, J. Aning for assistance with the FIA data analysis and T. Markus for assistance with the preparation of the figures. We also thank F. May and three anonymous referees for comments that improved the manuscript. Finally, we thank all of the FIA employees who collected the data that enabled us to test model predictions. 


\section{Author Contributions}

M.B.E., D.J.J., K.L.M.M., A.E.S and J.D.B. designed the study, M.B.E. and M.B. performed analytical model analyses, M.B.E., M.B., J.J., K.M.L.M. and A.E.S. developed code, performed and analysed model simulations, M.B.E., M.B., D.J.J and J.D.B. analysed field observation data, M.B.E. and J.D.B. wrote the first draft, after which all authors contributed to improving the manuscript.

\section{Declaration of Competing Interests}

The authors declare that they have no competing financial interests.

\section{References}

1. MacArthur, R. \& Levins, R. The limiting similarity, convergence and divergence of coexisting species. Am. Nat. 101, 377-385 (1967).

2. Tilman, D. Competition and biodiversity in spatially structured habitats. Ecology 75, 216 (1994).

3. Tilman, D. Resource Competition and Community Structure (Princeton University Press, Princeton, NJ, 1982).

4. Clark, J.S. Individuals and the variation needed for high species diversity in forest trees. Science 327, 1129-1132 (2010).

5. Allesina, S. \& Levine, J. A competitive network theory for species diversity. Proc. Nat. Acad. Sci. U.S.A. 108, 5638-5642 (2011).

6. Chesson, P. Mechanisms of maintenance of species diversity. Ann. Rev. Ecol. Evol. Syst. 31, 343-366 (2000). 
7. Barabás, G., Michalska-Smith, M.J. \& Allesina, S. The effect of intra- and interspecific competition on coexistence in multispecies communities. Am. Nat. 188, E1-E12 (2016).

8. Adler, P. HilleRisLambers, J. \& Levine, J. A niche for neutrality. Ecol. Lett. 10, 95-104 (2007).

9. Comita, L.S., Muller-Landau, H.C., Aguilar, S. \& Hubbell, S.P. Asymmetric density dependence shapes species abundances in a tropical tree community. Science 329, 330$332(2010)$.

10. Mangan, S.A. et al., Negative plant-soil feedback predicts tree-species relative abundance in a tropical forest. Nature 466, 752-756 (2010).

11. Johnson, D.J., Beaulieu, W.T., Bever, J.D. \& Clay, K. Conspecific negative density dependence and forest diversity. Science 336, 904-907 (2012).

12. LaManna, J.A., et al., Negative density dependence mediates biodiversity-productivity relationships across scales. Nature Ecol. Evol. 1, 1107-1115 (2017).

13. LaManna, J.A., et al., Plant diversity increases with the strength of negative density dependence at the global scale. Science 356, 1389-1392 (2017).

14. Hofbauer, J. \& Sigmund, K. Evolutionary games and population dynamics (Cambridge University Press, Cambridge, UK, 1998).

15. Allesina, S. \& Tang, S. Stability criteria for complex systems. Nature 483, 205-208 (2012).

16. Bascompte, J., Jordano, P. \& Olesen, J.M. Asymmetric coevolutionary networks facilitate biodiversity maintenance. Science 312, 431-433 (2006).

17. Chisholm, R.A. \& Muller-Landau, H. A theoretical model linking interspecific variation in density dependence to species abundances. Theor. Ecol. 4, 241-253 (2010).

18. Bever, J.D., Kristi, M.W. \& Antonovics, J. Incorporating the soil community into plant population dynamics: the utility of the feedback approach. J. Ecol. 85, 561-573 (1997). 
19. Bever, J.D. Soil community feedback and the coexistence of competitors: conceptual frameworks and empirical tests. New Phytol. 157, 465-473 (2003).

20. Kulmatiski, A., Heavilin, J. \& Beard, K.H. Testing predictions of a three-species plantsoil feedback model. J. Ecol. 99, 542-550 (2011).

21. Van der Putten, W.H., Van Dijk, C. \& Peters, B.A.M. Plant-specific soil-borne diseases contribute to succession in foredune vegetation. Nature 362, 53-56 (1993).

22. Revilla, T., Veen, G.F., Eppinga, M.B. \& Weissing, F.J. Plant-soil feedbacks and the coexistence of competing plants. Theor. Ecol. 6, 99-113 (2013).

23. Mack, K.M.L. \& Bever, J.D. Coexistence and relative abundance in plant communities are determined by feedbacks when the scale of feedback and dispersal is local. J. Ecol. 102, 1195-1201 (2014).

24. Levins, R. The qualitative analysis of partially specified systems. Ann. N.Y. Acad. Sci. 231, 123-138 (1974).

25. Wissel, C. A universal law of the characteristic return time near thresholds. Oecologia 65, 101-107 (1984).

26. Bennincà, E., Ballantine, B., Ellner, S.P. \& Huisman, J. Species fluctuations sustained by cyclic succession at the edge of chaos. Proc. Nat. Acad. Sci. U.S.A. 112, 6389-6394 (2015).

27. Klironomos, J.N. Feedback with soil biota contributes to plant rarity and invasiveness in communities. Nature 417, 67-70 (2002).

28. Neutel, A.M. et al. Reconciling complexity with stability in naturally assembling food webs. Nature 449, 599-602 (2007).

29. De Deyn, G.B. et al., Soil invertebrate fauna enhances grassland succession and diversity. Nature 422, 711-713 (2003). 
30. Mazzoleni, S. et al., Inhibitory and toxic effects of extracellular self-DNA in litter: a mechanism for negative plant-soil feedbacks? New Phytol. 205, 1195-1210 (2015).

31. Johnson, D.J., Beaulieu, W.T., Bever, J.D. \& Clay, K. Response to Comment on "Conspecific negative density dependence and forest diversity." Science 338, 469c (2012).

32. Dickie, I.A., Hurst, J.M. \& Bellingham, P.J. Comment on "Conspecific negative density dependence and forest diversity." Science 338, 469b (2012).

\section{Figure Legends}

Figure 1 | Conceptual representation of how the frequency-dependent feedback framework can be extended to the community level, for any number ( $n$ ) of plant species (here $n=5$ ). a) Plant species $\left(P_{i}\right)$ are modelled as frequencies within a community. The diagram includes trees from Laubwerk ${ }^{\circledR}$ Plant Kit 1 (www.laubwerk.com). b) Plant species exert conspecific $\left(\sigma_{i i}\right)$ and heterospecific $\left(\sigma_{i j}\right)$ effects, the magnitude of these effects (per capita) depending on the frequency of the exerting species. c) The resulting interaction network determines whether the community coexistence equilibrium is feasible; equilibrium frequencies are calculated by reduced determinants of the interaction matrix, $\mathbf{A}_{\mathbf{i}}$, the sum of which yield the community interaction coefficient, $I_{C}$. d) $I_{C}<0$ generates negative community-level feedback, and is a necessary condition for persistence of all plant species within the community. e) $I_{C}>0$ generates positive community-level feedback, and leads to competitive exclusion.

Figure $2 \mid 200$ runs of plant community assembly show that more species can stably coexist under stronger negative feedback. Filled line shows a quadratic fit assuming a minimum community size of 2 plant species $\left(y=39.00 x^{2}+12.52 x, R_{a d j}^{2}=0.86\right)$. 
Figure 3 | Effects of frequency-dependent feedback on plant community structure. a) Increasingly negative feedback increases the resilience of the coexistence equilibrium (measured as the absolute real part of the leading (and negative) eigenvalue ${ }^{27}$ ). b) Increasingly negative feedback increases species evenness within the stably coexisting community measured with Pielou's evenness index (i.e. the ratio between the actual and the maximum value of the Shannon-Wiener index) c) Even though community level feedback is determined by an analytical expression of the order $\sigma_{i j}^{n-1}$, the strength of average pair-wise feedback (i.e. of the order $\sigma_{i j}^{1}$ ) correlates well with the (rescaled) strength of this communitylevel feedback.

Figure $4 \mid$ Application of the theoretical framework to the United States Forest Service's Forest Inventory and Analysis database (see Supplementary Information section 5) reveals that observed patterns of negative frequency dependence in over 200,000 forest plots in the eastern United States are consistent with predictions from theoretical communities in which community-level feedback determines species richness. a) Map showing the locations of the 2 by 2-degree grid cells, and the community diversity observed in each cell (divided into 4 diversity classes: 5-15 spp. $(n=24), 15-25$ spp. $(n=36), 25-35$ spp. $(\mathrm{n}=22), \geq 35 \mathrm{spp} .(\mathrm{n}=31))$. b) Grid cells with higher community diversity are characterized by stronger negative feedback (coloured bars, Kruskal-Wallis H test, $X_{3,109}^{2}=$ $15.34, \mathrm{p}=0.0015)$, the variation in average pair-wise feedback strength being consistent with the range occurring in theoretical communities (grey bars: $\mathrm{n}_{5-15}=1059, \mathrm{n}_{15-25}=426, \mathrm{n}_{25-35}=272$, $\mathrm{n}>35=355$ ). Bars indicate means \pm 1 s.e.m. and different letters indicate differences at the $\mathrm{p}=$ 0.05 significance level (Kruskal-Wallis H test, $X_{7,2217}^{2}=1574, \mathrm{p}<1.10^{-323}$, followed by Dunn-Sidak post hoc tests). 


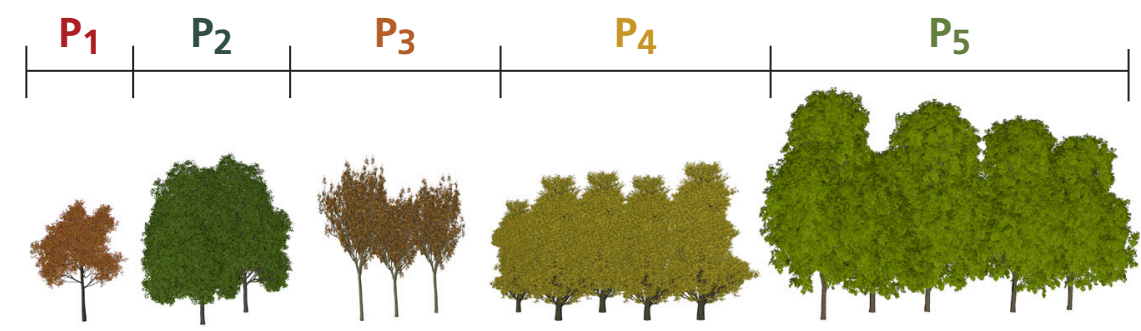

b

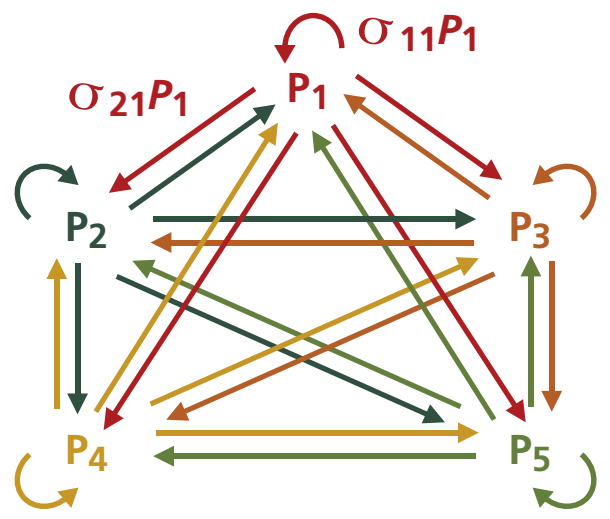

$\begin{gathered}\text { Feasibility : } 0<P_{i}=\frac{\operatorname{det} \mathbf{A i}}{\sum_{j=1}^{n} \operatorname{det} \mathbf{A j}}<1 \\ \text { Community feedback: } I_{c}=(-1)^{\mathrm{n}} \sum_{j=1} \operatorname{det} \mathbf{A j}\end{gathered} \quad \mathbf{A}_{3}=\left[\begin{array}{lllll}\sigma_{11} & \sigma_{12} 1 & \sigma_{14} & \sigma_{15} \\ \sigma_{11} & \sigma_{12} & \sigma_{14} & \sigma_{15} \\ \sigma_{11} & \sigma_{12} 1 & \sigma_{14} & \sigma_{15} \\ \sigma_{11} & \sigma_{12} & \sigma_{14} & \sigma_{15} \\ \sigma_{11} & \sigma_{12} & \sigma_{14} & \sigma_{15}\end{array}\right]$

$\mathrm{I}_{\mathrm{C}}<0$ : Negative feedback

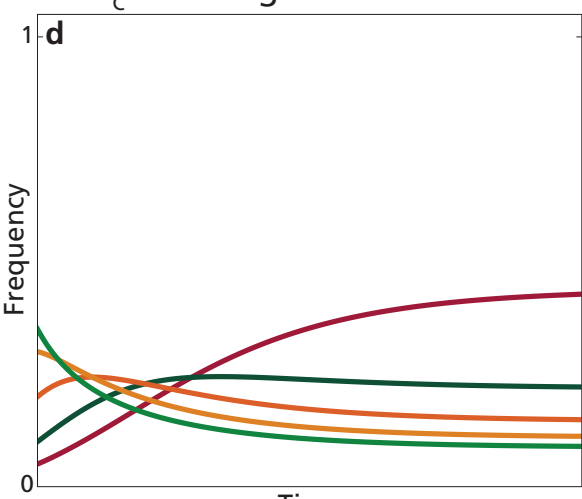

Time
$\mathrm{I}_{\mathrm{C}}>0$ : Positive feedback

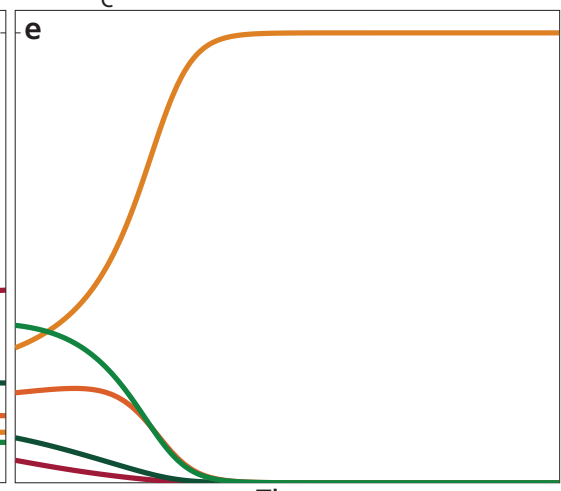




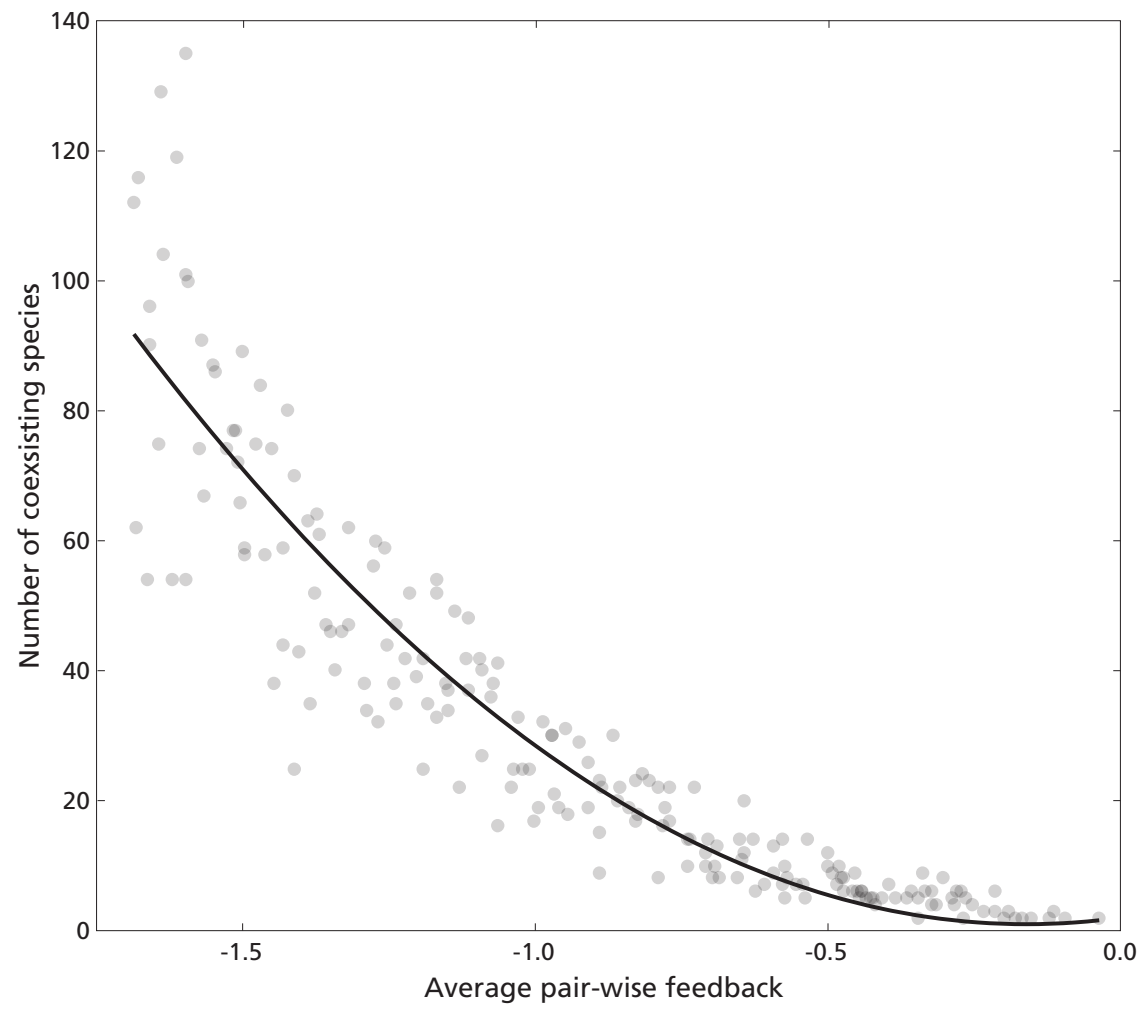



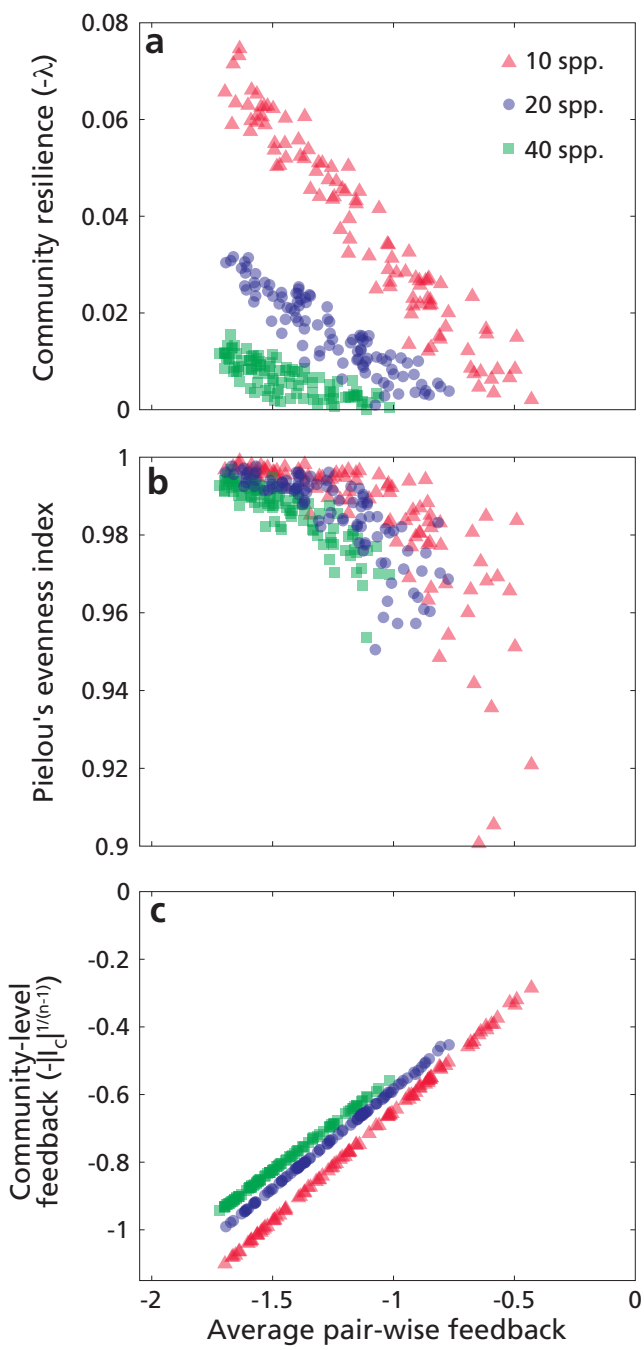
Supplementary Information

\section{TABLE OF CONTENTS}

1. Description of the model framework 2

Generalization of the two-species plant-soil feedback model $\quad 2$

Interpretation of the parameters quantifying frequency dependence 4

From 2-species density dynamics to $n$-species frequency dynamics: the replicator equation 6

Differences and similarities with the generalized Lotka-Volterra framework 9

2. Derivation and interpretation of the interaction coefficient $I_{S} \quad 13$

3. Existence and persistence conditions for $\boldsymbol{n}$-species coexistence $\quad 16$

Is the community interaction coefficient a sufficient criterion for species coexistence? $\quad 28$

Derivation of equation [S45] from equation [S40] 31

4. Species persistence despite instability of the coexistence equilibrium $\quad 34$

5. Field data and model parameterization 43

Characteristics of the dataset $\quad 43$

Calculation of the coefficients $\sigma_{i j}$ and model parameterization 46

Selection of grid cell size $\quad 56$

6. Robustness of the plant-soil feedback - species diversity relation $\quad 60$

7. Relaxing assumptions of zero-sum dynamics and fitness equality $\quad 64$

Equilibrium solution when relaxing the zero-sum assumption $\quad 64$

$\begin{array}{ll}\text { Introducing inherent fitness inequalities } & 66\end{array}$

$\begin{array}{ll}\text { Derivation of equation [S84] } & 71\end{array}$

$\begin{array}{ll}\text { References } & 74\end{array}$ 


\section{Description of the model framework}

In this section, we first describe how the model framework used in this study is a generalization of the two-species plant-soil feedback model developed by Bever et al. ${ }^{18}$. We then explain the meaning of the parameters quantifying frequency dependence. Subsequently, we show how the model equations project onto the well-known replicator equation when rewriting the equations to describe plant frequencies. We then explain the similarities and differences between the framework and the generalized Lotka-Volterra model.

\section{Generalization of the two-species plant-soil feedback model}

Bever et al. ${ }^{18}$, proposed a two-species model in which a standard exponential growth term describes the change in the number of plants:

$\frac{d N_{1}}{d t}=W_{1} N_{1}$

$\frac{d N_{2}}{d t}=W_{2} N_{2}$

In which $W_{i}$ is the average fitness of plant species $i$ (dimension: per time, $\mathrm{T}^{-1}$ ) and $N_{i}$ is the number of plants $\left(\# \mathrm{~N}_{\mathrm{i}}\right)$. Assuming a system of fixed area, these plant numbers describe plant densities. It is assumed that a functional soil community is present within the system, but that the composition of this community depends on the current densities of host plants ${ }^{18}$. Feedbacks can then occur in this system because average fitness of the host plants depends on the current composition of the soil community, which in turn responds to changes in plant community composition ${ }^{18}$. More specifically, $W_{1}$ and $W_{2}$ are defined as:

$$
\begin{aligned}
& W_{1}=\alpha_{11} S_{1}+\alpha_{12} S_{2} \\
& W_{2}=\alpha_{21} S_{1}+\alpha_{22} S_{2}
\end{aligned}
$$


In which $S_{i}$ is the proportion of soil effects attributable to plant species $i$ (dimensionless), and $\alpha_{i i}$ represents the sign and magnitude of direct soil effects initiated by host plants themselves $\left(\mathrm{T}^{-1}\right)$. Parameters $\alpha_{i j}\left(i \neq j, \mathrm{~T}^{-1}\right)$ represent the direction and magnitude of indirect soil effects on plant species $i$ that are initiated by competing plant species $j$. Here, we assume that plant species do not differ in their per capita effect on soil community composition, and that the soil community responds relatively quickly to changes in plant community composition. As noted $\mathrm{in}^{33}$, these assumptions lead to induced soil effects scaling linearly with the frequency of each plant species:

$$
\begin{aligned}
& S_{1}=\frac{N_{1}}{N_{1}+N_{2}} \\
& S_{2}=\frac{N_{2}}{N_{1}+N_{2}}
\end{aligned}
$$

Substituting equations [S3] into equations [S2] removes explicit soil community dynamics from the model, yielding a more general formulation of frequency-dependent effects emerging from plant-(soil-) environment interactions:

$$
\begin{aligned}
& \frac{d N_{1}}{d t}=\frac{\sigma_{11} N_{1}+\sigma_{12} N_{2}}{N_{1}+N_{2}} N_{1} \\
& \frac{d N_{2}}{d t}=\frac{\sigma_{21} N_{1}+\sigma_{22} N_{2}}{N_{1}+N_{2}} N_{2}
\end{aligned}
$$

Equations [S4] describe frequency-dependent effects with $\sigma_{i j}\left(\mathrm{~T}^{-1}\right)$ instead of $\alpha_{i j}$, to indicate that this system could describe not only plant-soil feedbacks, but also other types of frequency-dependent effects. It follows from equations [S4] that when all $\sigma_{i j}>0$, growth will be positive for both plant species. Exponential growth of biomass is a reasonable 
approximation for both herbaceous and woody seedlings growing for several weeks ${ }^{34}$, as is typically done in plant-soil feedback pot experiments ${ }^{18,21,35-39}$. When considering vegetation growth in ecosystems, however, finite amounts of space and resources will become limiting factors for total plant production and density. To enable model application across temporal and spatial scales, these effects can be included by introducing a crowding term similar to the classical Lotka-Volterra formulation for intra- and interspecific competition:

$$
\begin{aligned}
& \frac{d N_{1}}{d t}=\frac{\sigma_{11} N_{1}+\sigma_{12} N_{2}}{N_{1}+N_{2}} N_{1}-\rho_{1} \frac{N_{1}+c_{12} N_{2}}{K_{1}} N_{1} \\
& \frac{d N_{2}}{d t}=\frac{\sigma_{21} N_{1}+\sigma_{22} N_{2}}{N_{1}+N_{2}} N_{2}-\rho_{2} \frac{N_{2}+c_{21} N_{1}}{K_{2}} N_{2}
\end{aligned}
$$

In which $\rho_{i}$ is the response rate of species $i$ to crowding $\left(\mathrm{T}^{-1}\right), c_{i j}$ is an interspecific crowding coefficient describing the per capita effect of species $j$ on species $i$ (relative to the strength of intraspecific crowding, $\# \mathrm{~N}_{\mathrm{i}} \# \mathrm{~N}_{\mathrm{j}}^{-1}$ ), and $K_{i}$ controls the carrying capacity for species $i\left(\# \mathrm{~N}_{\mathrm{i}}\right)$. Equations [S5] are thus more general than equations [S1-S2] in terms of the potential mechanisms as well as the spatial and temporal scales that can be considered.

\section{Interpretation of the parameters quantifying frequency dependence}

Differences in resource uptake strategy and inherent fitness inequalities can be mimicked by setting the newly introduced parameters in equations [S5] differently for different species (e.g. $\left.{ }^{40}\right)$. To focus our current analysis on coexistence mediated through frequency-dependent effects on growth (rather than competitive interactions between species, for example), as introduced in the framework of Bever et al. ${ }^{18}$, we choose all $\rho_{i}=\rho_{j}=1, K_{i}=K_{j}=K$ and $c_{i j}=1$ (where $i \neq j$ ). Through these assumptions, we are thus investigating the stabilizing 
forces needed to enable coexistence of otherwise equally fit and equally competitive species through negative frequency-dependent feedback (e.g. ref. ${ }^{6}$ ). Hence, predictions made under these assumptions may lead to large discrepancies when compared to communities characterized by large differences in species' competitiveness. Hence, our analysis is aimed at contributing to a systematic approach to disentangling the relative importance of mechanisms contributing to the maintenance of plant community diversity. The rather specific choice of $\rho_{i}$ is not crucial for the results derived below (i.e. these results hold for $\rho_{i}=\rho_{j}=C$, with $C$ being any positive real constant), but $\rho_{i}=\rho_{j}=1$ yields a clear interpretation of the frequency-dependent effects $\sigma_{i j}$. Considering a single-species system, we can use equation [S5a] and solve $\left(\frac{d N_{1}}{d t}\right)_{N_{2}=0}=0$ to obtain:

$\hat{N}_{1}=\frac{K_{1}}{\rho_{1}} \sigma_{11}=K \sigma_{11}$

Where the hat indicates equilibrium. Equation [S6] shows that when $\sigma_{i i}=1$ (per unit of time), dynamics are not affected by frequency-dependent effects and the species (when growing by itself) will reach carrying capacity, which is then defined by $\frac{1}{\rho_{1}} K_{1}$. Alternatively, $0<\sigma_{i i}<1$ induces negative conspecific density dependence, reducing the species' equilibrium density. Finally, $\sigma_{i i}>1$ induces positive conspecific density dependence, and the species will be able to reach higher densities than its carrying capacity in the absence of these positive effects. Given the symmetry in the model equations [S5], the same interpretation applies to heterospecific effects. These (linear) effects of positive and 
negative density dependence on species' equilibria also emerge in more specific models describing plant density dynamics driven by plant-soil feedback ${ }^{19,22,41}$.

From 2-species density dynamics to $n$-species frequency dynamics: the replicator equation The described parameterization examines to what extent these frequency-dependent effects can generate coexistence between otherwise equivalent competitors. Therefore, comparisons between model predictions of coexistence can be compared to field data, and deviations between data and these predictions could provide indications of specific inherent fitness inequalities ${ }^{42}$ or equalizing mechanisms ${ }^{6}$, as well as the importance of alternative stabilizing mechanisms ${ }^{6}$. Inherent fitness inequalities can be included in the framework relatively easily (see Supplementary Information section 7). Analytical expressions for equilibrium densities in the model system described by equations [S5] can be obtained (Supplementary Information section 7), but such equilibrium solutions become more convenient when rewriting the model into species frequencies $\boldsymbol{P}$ (with $P_{i}=N_{i}\left(\sum_{j=1}^{n} N_{j}\right)^{-1}$ ) as we will show in Supplementary Information section 3. Moreover, frequencies are the appropriate metric when considering processes stabilizing coexistence ${ }^{8}$. For a two-species model (where $P_{2}=1-P_{1}$ ), applying the chain rule yields:

$\frac{d P_{1}}{d t}=\frac{d}{d t}\left(\frac{N_{1}}{N_{1}+N_{2}}\right)=\frac{\frac{d N_{1}}{d t}\left(N_{1}+N_{2}\right)-N_{1}\left(\frac{d N_{1}}{d t}+\frac{d N_{2}}{d t}\right)}{\left(N_{1}+N_{2}\right)^{2}}=\frac{P_{2} \frac{d N_{1}}{d t}-P_{1} \frac{d N_{2}}{d t}}{N_{1}+N_{2}}$

Substituting equations [S5] in [S7], we obtain:

$$
\frac{d P_{1}}{d t}=\left(\sigma_{11} P_{1}+\sigma_{12} P_{2}-\rho_{1} \frac{N_{1}+c_{12} N_{2}}{K_{1}}-\sigma_{21} P_{1}-\sigma_{22} P_{2}+\rho_{2} \frac{N_{1}+c_{22} N_{2}}{K_{2}}\right) P_{1} P_{2}
$$


Given our choices for $\rho_{i}, K_{i}$ and $c_{i j}$ (i.e. $K_{i}=K_{j}=K, \rho_{i}=\rho_{j}=\rho$ and $c_{12}=c_{21}=1$ ), we see that the two crowding terms become $\mp \rho \frac{\left(N_{1}+N_{2}\right)}{K}$ and cancel out. Hence, equation [S8] simplifies to:

$\frac{d P_{1}}{d t}=\left(\sigma_{11} P_{1}+\sigma_{12} P_{2}-\sigma_{21} P_{1}-\sigma_{22} P_{2}\right) P_{1} P_{2}$

And, by symmetry:

$\frac{d P_{2}}{d t}=\left(\sigma_{22} P_{2}+\sigma_{21} P_{1}-\sigma_{12} P_{2}-\sigma_{11} P_{1}\right) P_{1} P_{2}$

Equations [S9] now describe the growth dynamics of the two plant species, expressed as frequencies, $P_{i}$ (with: $\sum_{i=1}^{n} P_{i}=1$ ). Changes in plant species frequency modify their growth rates, feeding back to further changes in frequencies. Combining equations [S2] and [S3], this frequency-dependent feedback is described by:

$$
w_{i}=\sum_{j=1}^{n} \sigma_{i j} P_{j}
$$

Inserting these descriptions of feedback (equation [S10]) into equations [S9], we obtain:

$$
\begin{aligned}
& \frac{d P_{1}}{d t}=\left(w_{1}-w_{2}\right) P_{1} P_{2}=P_{1}\left(w_{1}-w_{1} P_{1}-w_{2} P_{2}\right) \\
& \frac{d P_{2}}{d t}=\left(w_{2}-w_{1}\right) P_{1} P_{2}=P_{2}\left(w_{2}-w_{1} P_{1}-w_{2} P_{2}\right)
\end{aligned}
$$

Equations [S11] can be generalized to describe the growth dynamics of any number of plant species, $n$, in a community:

$$
\frac{d P_{i}}{d t}=P_{i}\left(w_{i}-\sum_{j=1}^{n} w_{j} P_{j}\right)
$$


Where we note that in a community with $n$ species, there are now $n$ - 1 degrees of freedom, because $P_{n}=1-\sum_{i=1}^{n-1} P_{i}$. Equation [S12] is the well-known replicator equation, which has been extensively studied in evolutionary and ecological game theory (e.g. ${ }^{6,14,43,44}$ ) and applied in economics $\left(\right.$ e.g. ${ }^{45}$ ). As shown in Supplementary Information sections 2 and 3, equilibrium points for dynamical systems described by equation [S12] can easily be derived. It should be noted however, that the link between equations [S5] and the replicator equation is not unique. The last negative terms of the right-hand side of equations [S5] can be replaced with any term $f(\vec{N}) N_{i}$ : as long as $f(\vec{N})$ is the same for each species, the dynamics of the corresponding system still project on the same set of replicator equations ([S12]). This means that also the dynamics of the original exponential growth model proposed by Bever et al. ${ }^{18}$ (equations $[\mathrm{S} 1], f(\vec{N})=0$ ) can be rewritten in the form of the replicator equation. In this latter case, however, even species at low frequencies may still increase exponentially in numbers. Similarly, in cases where a generic mortality term is included $(f(\vec{N})=m)$, even species with high frequencies may approach a population size of zero if $m>\max \left(\sigma_{i j}\right)$. More generally, an equilibrium point with asymptotically stable frequencies of each species does not provide direct information about density dynamics, which may still be changing over time while remaining at equilibrium frequency. It is thus relevant to specify that we analyse the replicator equation in this study as being a projection of the underlying model described by the plant density equations [S5] and the associated interpretation of $\sigma_{i j}$, the parameters quantifying frequency dependence. 
Differences and similarities with the generalized Lotka-Volterra framework

Hofbauer ${ }^{43}$ showed that (apart from a time conversion constant) the replicator equation (equation [S12]) also provides a representation of the generalized Lotka-Volterra model:

$$
\frac{d x_{i}}{d t}=x_{i}\left(a_{i, 0}+\sum_{j=1}^{n} a_{i, j} x_{j}\right)
$$

In which $x_{i}$ generally represent densities of different species. More specifically, an $n$-species generalized Lotka-Volterra model (Equation [S13]) can be projected onto a system of $n+1$ replicator equations when dynamics are rewritten to describe species frequencies $(y)$ by means of the following transformation ${ }^{43,46}$ :

$$
y_{i}=\frac{x_{i}}{\sum_{i=0}^{n} x_{i}}
$$

In which $x_{0}=1$. In this transformed system, the extra variable $y_{0}$ establishes the relationship between species frequency and density. Following ${ }^{43,46}$, we set:

$$
y_{0}=\frac{x_{0}}{\sum_{i=0}^{n} x_{i}}=\frac{1}{\sum_{i=0}^{n} x_{i}}
$$

Starting with a two-species generalized Lotka-Volterra model, we can rewrite equation [S13] through application of the chain rule:

$\frac{d y_{1}}{d t}=\frac{d}{d t}\left(\frac{x_{1}}{x_{0}+x_{1}+x_{2}}\right)=\frac{\frac{d x_{1}}{d t}\left(x_{0}+x_{1}+x_{2}\right)-x_{1}\left(\frac{d x_{0}}{d t}+\frac{d x_{1}}{d t}+\frac{d x_{2}}{d t}\right)}{\left(x_{0}+x_{1}+x_{2}\right)^{2}}$

Using equation [S15], we can write: 


$$
\frac{d y_{1}}{d t}=\frac{\frac{d x_{1}}{d t}}{x_{0}+x_{1}+x_{2}}-\frac{x_{1}\left(\frac{d x_{0}}{d t}+\frac{d x_{1}}{d t}+\frac{d x_{2}}{d t}\right)}{\left(x_{0}+x_{1}+x_{2}\right)^{2}}=y_{0}\left(\frac{d x_{1}}{d t}-y_{0} x_{1}\left(\frac{d x_{0}}{d t}+\frac{d x_{1}}{d t}+\frac{d x_{2}}{d t}\right)\right)
$$

Filling in equation $[\mathrm{S} 13]$ then yields:

$$
\frac{d y_{1}}{d t}=y_{0}\left(x_{1}\left(\sum_{j=0}^{2} a_{1, j} x_{j}\right)-y_{0} x_{1}\left(\sum_{i=0}^{2} x_{i}\left(\sum_{k=0}^{2} a_{i, k} x_{k}\right)\right)\right)
$$

Which simplifies to the following replicator equation:

$$
\frac{d y_{1}}{d t}=\frac{1}{y_{0}}\left(y_{1}\left(\sum_{j=0}^{2} a_{1, j} y_{j}\right)-y_{1}\left(\sum_{i=0}^{2} y_{i}\left(\sum_{k=0}^{2} a_{i, k} y_{k}\right)\right)\right)
$$

Equation [S19] is similar to the previously derived equation [S12], which can be shown by introducing:

$$
w_{i}=\sum_{j=0}^{n} a_{i j} y_{j}
$$

Combining equations [S19] and [S20] the replicator equation for a plant community of any size can now be written as:

$$
\frac{d y_{i}}{d t}=\frac{y_{i}}{y_{0}}\left(w_{i}-\sum_{j=0}^{n} w_{j} y_{j}\right)
$$

The corresponding interaction matrix now takes the form ${ }^{43,46}$ :

$$
\mathbf{A}_{r e p}=\left[\begin{array}{cccc}
0 & 0 & \ldots & 0 \\
a_{10} & a_{11} & \ldots . & a_{1 n} \\
a_{20} & a_{21} & \ldots . & a_{2 n} \\
\ldots & \ldots & \ldots . & \ldots \\
a_{n 0} & a_{n 1} & \ldots . & a_{n n}
\end{array}\right]
$$

Equation [S22] suggests that the extra variable in system of $n+1$ replicator equations can be seen as unoccupied space, which can be colonized by the plant species at a rate proportional 
to their relative growth rate $a_{i 0}$. As long as the total amount of space occupied by the plant community is constant, changes in community composition will not affect the dynamics of the extra variable (as implied by the row of zeros in equation [S22]). In this system overall feedback $^{24,47}$ reduces to det $\mathbf{A}_{\text {rep }}=0$. In subsequent sections, we use $\operatorname{det}[\mathbf{A}]$ in some of the derivations, which are therefore restricted to the system previously described in this section (equation [S12]). Moreover, in the latter system (equation [S21]) frequency dependence is described by parameters $a_{i, j}$, which quantify intra- and interspecific competition in the generalized Lotka-Volterra model. Therefore, the key difference between the projections of the two frameworks onto the replicator equation is related to the processes giving rise to frequency dependence. The generalized plant-soil feedback model of Bever et al. ${ }^{18}$ assumes that density dependence affects the growth rate of competing plant species, while assuming that these species are equally competitive for resources constraining their carrying capacity. In contrast, the generalized Lotka-Volterra model assumes that plant species' growth rates are equal, but density-dependent effects arise through differences in intraspecific and interspecific interaction strengths, for example caused by different resource utilization strategies $^{7}$. Levins ${ }^{48}$ derived how the concept of resource overlap created symmetrical interaction matrices within the generalized Lotka-Volterra model. Subsequently, Goh ${ }^{49}$ showed that for this specific type of interaction matrices, det $\mathbf{A}>0$, provides a necessary and sufficient condition for coexistence. This condition, however, no longer holds for the asymmetric interaction matrices considered here.

In this study we applied our theoretical framework to examine to what extent frequencydependent effects on (seedling) growth could drive observed patterns of species coexistence. Therefore, we assigned the model parameter values in equation [S12] based on their meaning 
in the generalized model of frequency dependence (equation [S5]) derived from Bever et al. ${ }^{18}$, using data on (adult) frequency-dependent seedling growth ${ }^{11,31}$ (see Supplementary Information section 5). 


\section{Derivation and interpretation of the interaction coefficient $I_{S}$}

In this section we will derive the interaction coefficient $I_{S}$ for a two-species model. The interaction coefficient $I_{S}$ was previously presented in $^{18}$. Using equation [S11], we can write the two-species model as:

$$
\begin{aligned}
& \frac{d P_{1}}{d t}=P_{1}\left(w_{1}-w_{1} P_{1}-w_{2} P_{2}\right)=P_{1}\left(w_{1}-w_{1} P_{1}-w_{2}\left(1-P_{1}\right)\right) \\
& \frac{d P_{2}}{d t}=P_{2}\left(w_{2}-w_{1} P_{1}-w_{2} P_{2}\right)=\left(1-P_{1}\right)\left(w_{2}-w_{1} P_{1}-w_{2}\left(1-P_{1}\right)\right)=-\frac{d P_{1}}{d t}
\end{aligned}
$$

Thus, finding equilibrium points only requires solving equation [S23a], which yields:

$$
P_{1}=0 \vee-I_{S} P_{1}^{2}+\left(I_{S}+E_{2}\right) P_{1}-E_{2}=0
$$

In which:

$$
\begin{aligned}
& I_{S}=\sigma_{11}-\sigma_{12}-\sigma_{21}+\sigma_{22} \\
& E_{2}=\sigma_{22}-\sigma_{12}
\end{aligned}
$$

In which $I_{S}$ is the community interaction coefficient ${ }^{18}$, and $E_{2}$ represents the species-specific feedback that is exerted by species 2 . If $E_{2}$ is positive, plant species 1 will not be able to invade an environment dominated by plant species 2 . The quadratic equation in [S24] then yields two solutions:

$$
P_{1}=\frac{I_{S}+E_{2}+\sqrt{\left(I_{S}+E_{2}\right)^{2}-4 I_{S} E_{2}}}{2 I_{S}}=1 \vee P_{1}=\frac{I_{S}+E_{2}-\sqrt{\left(I_{S}+E_{2}\right)^{2}-4 I_{S} E_{2}}}{2 I_{S}}=\frac{E_{2}}{I_{S}}
$$

Equation sets [S24] and [S26] show that the system has three equilibrium points, two of these points being at the boundaries of the state space, i.e. $P_{1}=0$ and $P_{1}=1$. Whether there is an internal equilibrium where both species have non-zero frequency depends on the signs of $E_{2}$ and $I_{S}$. This yields four possible situations (Fig. S1). 

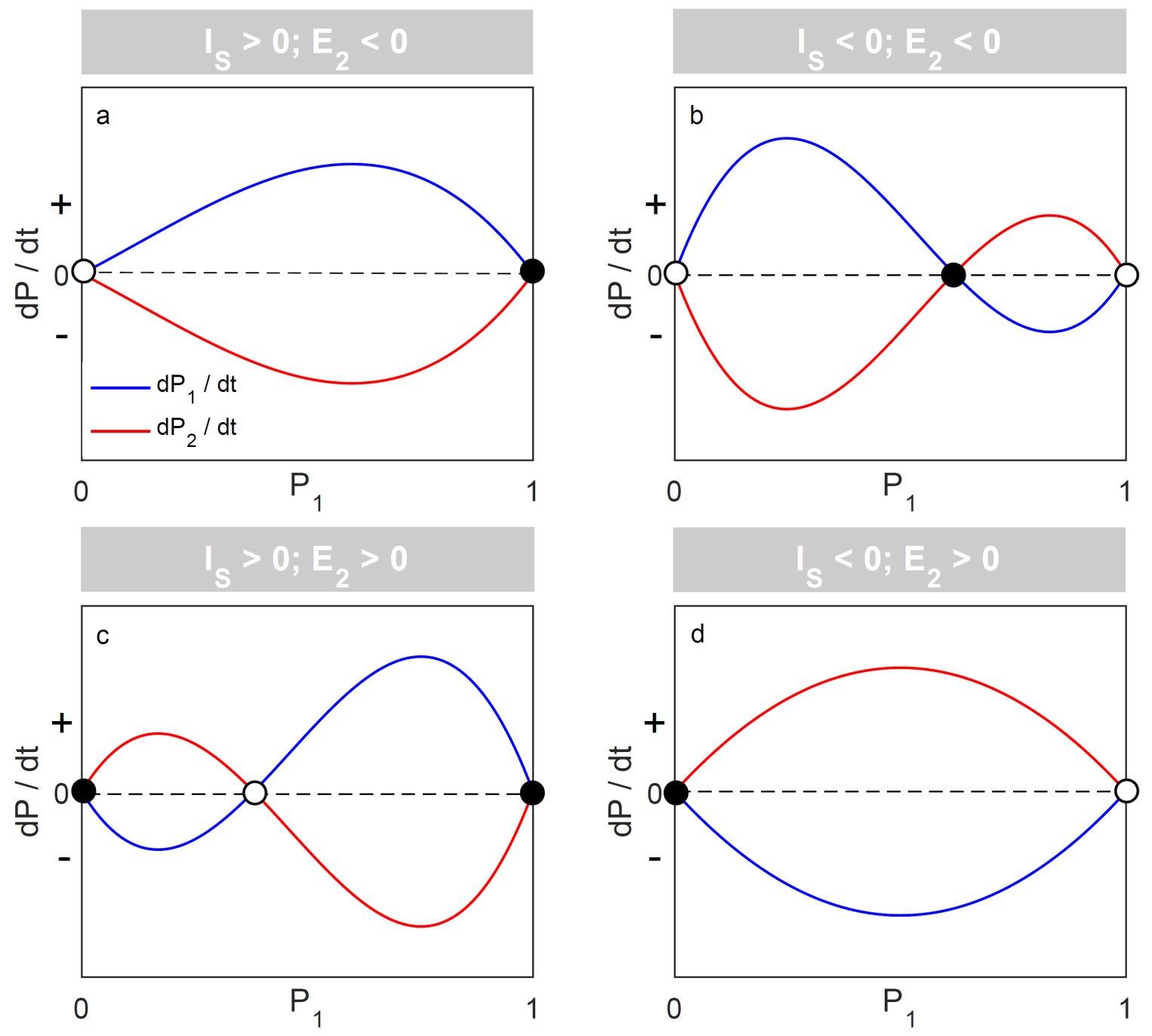

Supplementary Figure S1 | The four potential outcomes between two plant species that experience

frequency-dependent feedbacks. In all four panels, the dotted line indicates $\frac{d P_{1}}{d t}=\frac{d P_{2}}{d t}=0$. Stable equilibria are marked by filled circles, unstable equilibria are marked by open circles. Two equilibrium points are always present at the boundaries of the state space $\left(P_{1}=0\right.$ and $\left.P_{1}=1\right)$. The existence and stability of the internal equilibrium depends on the signs of $I_{S}$ (equation [S25a]) and $E_{2}$ (equation [S25b]). Importantly, stable coexistence requires $I_{S}<0$, meaning a negative feedback ${ }^{18}$. 
Existence of the internal equilibrium (within the biologically relevant state space) requires that $E_{2}$ and $I_{S}$ have the same sign. When $E_{2}, I_{S}>0$, neither species can recover when rare, meaning that the internal equilibrium is unstable. Also under these conditions, there is an equilibrium point with non-zero frequencies for both plant species. However, even small perturbations from this equilibrium point will be amplified; the fitness of the species perturbed below its equilibrium frequency decreases, leading to a further decrease in this species' frequency. This unstable nature of the equilibrium point is reflected by $I_{S}>0$, which generates this positive feedback within the system. Due to this positive feedback mechanism, the system will move away from the equilibrium point, until one species is excluded (Fig. S1c). Hence, this kind of internal equilibrium points is referred to as an unstable equilibrium. In contrast, perturbations of a stable equilibrium point will be damped through negative feedback within the system (Fig. S1b). In this latter case, the fitness of the species perturbed below its equilibrium frequency increases, leading to a subsequent increase in frequency that counteracts the initial perturbation. In the system discussed here, $I_{S}<0$, generates such a negative feedback. This means that stable coexistence requires that $E_{2}, I_{S}<0$ (Fig. S1). 


\section{Existence and persistence conditions for $\boldsymbol{n}$-species coexistence}

In this section, we will first briefly recap a result presented $\mathrm{in}^{33}$, where it was shown that there is a relatively simple analytical description of the internal coexistence equilibrium for any number of species that interact as described by equation [S12]. We note that this analytical result has also been obtained within other applications of the replicator equation ${ }^{14}$, and that obtaining a solution is not critically depending on the assumption of zero-sum dynamics of plant frequencies (as we will later demonstrate in Supplementary Information section 7). We then show that this analytical solution not only yields densities of all species at equilibrium, but it also reveals that the hitherto considered pair-wise interaction coefficient $I_{S}{ }^{18}$ is a specific (2-species) case of the more general community interaction coefficient $I_{C}$, which governs stability of $n$ species comprising a plant community. More specifically, we will show that stability of the internal coexistence equilibrium requires $I_{C}<0$. In Supplementary Information section 4, we will then continue with showing that when the $I_{C}$ criterion is met, species persistence may be possible even when the internal equilibrium is linearly unstable (i.e. the real part of the leading eigenvalue of the Jacobian, evaluated at the internal coexistence equilibrium, is positive).

Recalling that the dynamics of each species are described by:

$$
\frac{\mathrm{d} P_{i}}{\mathrm{~d} t}=P_{i}\left(w_{i}-\sum_{j=1}^{n} w_{j} P_{j}\right)
$$

Where $w_{i}=\sum_{j=1}^{n} \sigma_{i j} P_{j}$ (as in equation [S10]), we can investigate whether a coexistence equilibrium (i.e. with all $P_{i}>0$ ) exists by equating the second factor on the right-hand side of 
[S27] to zero for each species $i$. We obtain $\hat{w}_{i}-\sum_{j=1}^{n} \hat{w}_{j} \hat{P}_{j}=0$, from which we can infer that $\hat{w}_{i}=\sum_{j=1}^{n} \hat{w}_{j} \hat{P}_{j}$ for all species, meaning:

$\hat{w}_{1}=\hat{w}_{2}=\hat{w}_{i}=\ldots=\hat{w}_{n}=\hat{w}$

Substituting the expression for $\hat{w}_{i}$ (equation [S10]) into equation [S28] we obtain:

$$
\hat{w}=\sum_{j=1}^{n} \sigma_{i j} \hat{P}_{j}
$$

In words, equation [S29] denotes that at the coexistence equilibrium, all species have equal fitness (also referred to as equal per capita growth rate $^{6}$ ). Rewriting equation [S29] in matrix notation, i.e. for all $i=1,2 \ldots n$, we can obtain the equilibrium frequencies $\hat{P}_{i}$ for all $n$ species by solving the following matrix equation:

$\mathbf{A} \times \widehat{\boldsymbol{P}}=\left[\begin{array}{cccc}\sigma_{11} & \sigma_{12} & . . & \sigma_{1 n} \\ \sigma_{21} & . . & . . & . . \\ . . & . . & . . & . . \\ \sigma_{n 1} & . . & . . & \sigma_{n n}\end{array}\right] .\left[\begin{array}{c}\hat{P}_{1} \\ \hat{P}_{2} \\ . . \\ \hat{P}_{n}\end{array}\right]=\hat{w} . \mathbf{1}$

Here, the matrix A specifies the $n \times n$ frequency-dependent effects within the network. As we will show below, the structure of this matrix governs equilibrium stability, similar to the way in which the so-called interaction matrix (i.e. the Jacobian matrix) governs stability in food webs (e.g. used $\mathrm{in}^{50}$ ). Even though $\mathbf{A}$ is not the Jacobian matrix, due to its similar role in governing network dynamics we will refer to the matrix $\mathbf{A}$ as an interaction matrix in this study, following e.g. ${ }^{7}$. In equation [S30], 1 specifies a column vector of length $n$, and $\widehat{\mathbf{P}}$ is a vector describing the frequencies of all $n$ species at equilibrium. As $\sigma_{i j} \geq 0$ (see 
Supplementary Information section 1), it follows that $\hat{w} \geq 0$, which means that we can use Cramer's rule to solve equation [S30], yielding:

$\hat{P}_{i}=\hat{w} \frac{\operatorname{det} \mathbf{A}_{i}}{\operatorname{det} \mathbf{A}}$

Where $\mathbf{A}_{i}$ indicates a matrix equal to $\mathbf{A}$, except for the $i^{t h}$ column, which is replaced by a column vector of ones. Note that in the case where $\operatorname{det} \mathbf{A}=0$, no net feedback emerges in the model, which means that an equilibrium solution for this model (in which frequencydependent feedback is the sole driving factor of species dynamics) cannot be obtained. Because $\hat{w}$ is a function of $\hat{P}$ (see equation [S10]), equation [S31] is not a useful solution yet, as elements of $\hat{P}$ occur on both sides of the equation. A useful solution can be found by recalling that plant dynamics are modelled as frequencies (i.e. $\sum_{i=1}^{n} P_{i}=1$ ), and thus:

$\sum_{i=1}^{n} \hat{P}_{i}=\sum_{i=1}^{n} \frac{\hat{w} \operatorname{det} \mathbf{A}_{i}}{\operatorname{det} \mathbf{A}}=\hat{w} \frac{\sum_{i=1}^{n} \operatorname{det} \mathbf{A}_{i}}{\operatorname{det} \mathbf{A}}=1$

From equation [S32], it follows that:

$$
\sum_{j=1}^{n} \operatorname{det} \mathbf{A}_{j}=\frac{\operatorname{det} \mathbf{A}}{\hat{w}}
$$

Replacing [S33] into equation [S31] we obtain:

$$
\hat{P}_{i}=\frac{\operatorname{det} \mathbf{A}_{i}}{\sum_{j=1}^{n} \operatorname{det} \mathbf{A}_{j}}=\frac{\operatorname{det} \mathbf{A}_{i}}{\psi_{C}^{(n)}}
$$

In which $\psi_{C}^{(n)}$ is the sum of feedback effects experienced within the community of $n$ plant species. Note that $I_{S}$ as previously derived in ${ }^{18,19}$ is the two-species case of $\psi_{C}^{(n)}$. From 
equation [S34] it follows that $\psi_{C}^{(n)}$ is of the order $\sigma^{n-1}$. This is relevant, because in the twospecies case hitherto considered, $\psi_{C}^{(n)}$ consisted of first order terms (i.e. $\psi_{C}^{(2)}=I_{S}=\sigma_{i i}-\sigma_{i j}-\sigma_{j i}$ $\left.+\sigma_{j j}\right)$, but this turns out to be a specific case. Equation [S34] reveals that there is a relatively simple relation between the structure of the interaction matrix and the coexistence equilibrium.

With the solution described by equation [S34] we can now formalize the feasibility condition (e.g. used $\mathrm{in}^{51}$ ) for the $n$ species coexistence equilibrium:

$0<\frac{\operatorname{det} \mathbf{A}_{i}}{\sum_{j=1}^{n} \operatorname{det} \mathbf{A}_{j}}<1 \quad$ for $i=1,2, \ldots, n$

Note that verification of the feasibility condition (equation [S35]) only requires the calculation of the $n$ determinants of the interaction matrix $\mathbf{A}$ following single-column substitution. Once feasibility has been verified, the coexistence equilibrium densities $\hat{P}_{i}$ can be inserted into the Jacobian matrix of the system:

$$
J=\left[\begin{array}{cccc}
\frac{\partial F_{1}}{\partial P_{1}} & \frac{\partial F_{1}}{\partial P_{2}} & . . & \frac{\partial F_{1}}{\partial P_{n}} \\
\frac{\partial F_{2}}{\partial P_{1}} & . . & . . & . . \\
\frac{\partial F_{n}}{\partial P_{1}} & . . & . . & . . \\
\frac{\partial F_{n}}{\partial P_{n}}
\end{array}\right]
$$

With $F_{i}=P_{i}\left(w_{i}-\sum_{j=1}^{n} w_{j} P_{j}\right)$. The leading eigenvalue of this Jacobian matrix (i.e. the eigenvalue that has the maximal real part) evaluated at the coexistence equilibrium point $\hat{\mathbf{P}}=\left\{\hat{P}_{1}, \hat{P}_{2}, \ldots, \hat{P}_{n}\right\}$, determines its local stability. When the real part of the leading eigenvalue 
is negative, the real parts of all eigenvalues are negative, meaning that the perturbed state variables $\mathbf{P}(t)$ will return to the equilibrium state $\hat{\mathbf{P}}$ over time following:

$$
\mathbf{P}(t)-\hat{\mathbf{P}}=\sum_{j=1}^{n} \sum_{i=1}^{n-1} L_{i} e^{\lambda_{i}\left(t-t_{0}\right)} v_{i, j}
$$

Equation [S37] provides a general description, in that any perturbation $\boldsymbol{L}$ of a system of $n$ variables (exerted at time $t=t_{0}$ ) can be expressed as a linear combination of $n-1$ linearly independent eigenvectors $\vec{v}_{i}$, each of these components of the perturbations growing or decaying over time at a rate $\lambda_{i}\left(\mathrm{~T}^{-1}\right)$. Indeed, all $\operatorname{Re}\left[\lambda_{i}\right]<0$ ensures that each of the $n$ perturbation terms $L_{i} \vec{v}_{i}$ in equation [S37] decays exponentially over time, and the system returns to $\hat{\mathbf{P}}$. It is important to note here that perturbed system states still need to obey $\sum_{j=1}^{n} P_{j}(t)=1$, which explains why perturbations only contain $n-1$ degrees of freedom that can be decomposed in $n-1$ linearly independent eigenvectors. These latter eigenvectors are the socalled relevant eigenvectors in the analysis ${ }^{52}$. As the above condition needs to hold for any perturbation, we can examine the fate of a specific perturbation:

$$
\mathbf{L}=\left[L_{1}, L_{2}, \ldots, L_{n-1}\right]=\left[0,0, \ldots, L_{k}, \ldots, 0\right]
$$

In words, equation [S38] represents a perturbation that follows the $k^{\text {th }}$ relevant eigenvector. The resulting system dynamics in response to this specific perturbation can then be written as:

$$
\sum_{j=1}^{n} P_{j}(t)-\sum_{j=1}^{n} \hat{P}_{j}=\sum_{j=1}^{n} L_{k} e^{\lambda_{k}\left(t-t_{0}\right)} v_{k, j}=L_{k} e^{\lambda_{k}\left(t-t_{0}\right)} \sum_{j=1}^{n} v_{k, j}=0
$$


It follows that equation [S39] can only be fulfilled if $\sum_{j=1}^{n} v_{k, j}=0$. Given the linear independence of the $n-1$ relevant eigenvectors, equation [S39] applies to all the relevant eigenvectors. We also note for these relevant eigenvectors that $\sum_{j=1}^{n}\left|v_{k, j}\right| \neq 0$. It also follows that the $n^{\text {th }}$ eigenvector of the Jacobian matrix can only be linearly independent from the $n-1$ relevant eigenvectors if $\sum_{j=1}^{n} v_{n, j} \neq 0$, which means that system perturbations (partly) described by this vector will not obey $\sum_{j=1}^{n} P_{j}(t)=1$, and should therefore be discarded in calculations of equilibrium stability. Below we will derive a quantitative expression for the irrelevant eigenvalue that is associated with this irrelevant eigenvector. Hence, when referring to the leading eigenvector in the remaining analyses, we refer to the leading relevant eigenvector, i.e. the eigenvalue with the maximal real part of the set of $n-1$ eigenvectors that obey $\sum_{j=1}^{n} v_{k, j}=0$

We will now turn to the question of how $\psi_{C}^{(n)}$ relates to the stability of the community coexistence equilibrium. This part of the analysis will focus on the ability of species to recover (i.e. increase in frequency) when rare (e.g. used $\mathrm{in}^{41}$ ), also referred to as an invasion analysis (e.g. used in ${ }^{6,22}$ ). Global stability of the community coexistence equilibrium requires that all species are able to recover when rare. Previous work has shown that local stability of coexistence equilibria may be possible in the absence of uniform recovery ability, but this type of dynamics requires disproportional effects of at least one species ${ }^{22}$. Because in the current model formulation all species effects are proportional to their frequency (equation 
[S10]), the recovery condition derived below comprises a necessary condition for community coexistence. We will derive the recovery conditions for a plant species $n$ invading a system of $n-1$ species. Therefore, the description of the system's dynamics increases from $n-1$ to $n$ equations. Effects of the invading species on the other species appear in the $n^{\text {th }}$ column of the interaction and Jacobian matrices. Effects of the other species on the invading species appear in the $n^{\text {th }}$ row of the interaction and Jacobian matrices. The dynamics of the invading species are described by equation [S27], which can be rewritten for $n$ species as a third order polynomial in $P$ :

$$
\frac{\mathrm{d} P_{i}}{\mathrm{dt}}=P_{i}\left(\sum_{j=1}^{n} \sigma_{i j} P_{j}-\sum_{j=1}^{n} \sum_{k=1}^{n} \sigma_{j k} P_{j} P_{k}\right)
$$

We introduce $\widetilde{\mathbf{P}}=\left\{\breve{P}_{1}, \breve{P}_{2}, \ldots, \breve{P}_{n-1}, \breve{P}_{n}=0\right\}$ to specify the system state that $P_{n}$ needs to be able to invade, where the first $n$ - 1 elements are the equilibrium solutions of the system of equations [S40] for these $n$-1 species. We write this equilibrium state as $\breve{\mathbf{P}}=\left\{\breve{P}_{1}, \breve{P}_{2}, \ldots, \breve{P}_{n-1}\right\}$. Noting that the first term on the right hand side in equation [S40] prevents zero order terms in the polynomial, we obtain the following, general result for the $n^{\text {th }}$ row of the Jacobian matrix: $\frac{\partial}{\partial P_{j}}\left(\frac{\mathrm{d} P_{n}}{\mathrm{dt}}\right)_{\mathbf{P}=\widetilde{\mathbf{P}}}=0$ for $j \neq n$

Where $\mathbf{P}=\widetilde{\mathbf{P}}$ specifies that the invasion (recovery) potential of $P_{n}$ is evaluated at $\widetilde{\mathbf{P}}$. From here we refer to the interaction matrix of the $n$ species system as $\mathbf{A}$, and the interaction matrix of the $n-1$ species system as $\breve{\mathbf{A}}$, which is associated with the $n$-1 non-zero frequencies for the plant species present in the community before invasion. From equation [S41], it becomes clear that evaluating the Jacobian in $\widetilde{\mathbf{P}}$ (with $P_{n}=0$ and all other plant densities as in $\breve{\mathbf{P}}$ ) all the entries on the $n^{\text {th }}$ row of the Jacobian become zero, except the entry in the $n^{\text {th }}$ column (i.e. 
the derivative of the growth equation to $P_{n}$ itself). For the $n-1$ species system, the eigenvalues are obtained from the characteristic equation:

$\operatorname{det}[\breve{J}-\breve{\lambda I}]=0$

In which $\breve{I}$ is an identity matrix of $n-1$ rows and columns. It follows from equations [S36] and [S41] that we can write for the $n$ species system:

$$
\operatorname{det} J=\operatorname{det}\left[\begin{array}{cccc}
\frac{\partial F_{1}}{\partial P_{1}} & \frac{\partial F_{1}}{\partial P_{2}} & . . & \frac{\partial F_{1}}{\partial P_{n}} \\
\frac{\partial F_{2}}{\partial P_{1}} & . . & . . & . . \\
. . & . . & . . & . \ddot{F}_{n} \\
0 & 0 & . . & \frac{\partial F_{n}}{\partial P_{n}}
\end{array}\right]=\operatorname{det}\left[\begin{array}{cccc}
\frac{\partial P_{n}}{\partial P_{1}} & 0 & . . & 0 \\
\frac{\partial F_{1}}{\partial P_{1}} & . . & . . \\
\frac{\partial F_{n-1}}{\partial P_{n}} & \frac{\partial F_{n-1}}{\partial P_{1}} & . . & \frac{\partial F_{n-1}}{\partial P_{n-1}}
\end{array}\right]=\frac{\partial F_{n}}{\partial P_{n}} \operatorname{det}[\breve{J}]
$$

Where we interchanged $n$ pairs of rows and $n$ pairs of columns in the Jacobian. This total of $2 n$ interchanges is always an even number, and therefore does not lead to a sign change of the rearranged determinant. Hence, for the system with the $n^{\text {th }}$ species invading, the eigenvalues are obtained from the characteristic equation:

$$
\operatorname{det}[J-\lambda I]=\left(\frac{\partial F_{n}}{\partial P_{n}}-\lambda_{n}\right) \operatorname{det}[\breve{J}-\breve{\lambda I}]=0
$$

From equation [S44] it is clear that either the first or the second factor in the product needs to be zero, the latter group of solutions for $\breve{\lambda}$ already described by equation [S42]. Thus, this means that all relevant eigenvalues of the $n-1$ system are also eigenvalues of the system in which the $n^{\text {th }}$ species is invading. These relevant eigenvalues are thus not influenced by $P_{n}$. To further illustrate this notion, we recall that evaluating the Jacobian of the $n-1$ species system evaluated at $\breve{\mathbf{P}}$ yields $n-2$ relevant eigenvalues, meaning that the associated eigenvectors obey $\sum_{k=1}^{n-1} v_{j k}=0$. As relevant eigenvalues in the $n$-species system now need to 
obey $\sum_{k=1}^{n} v_{j k}=0$, it follows that $v_{n k}=0$ for all $n$-2 relevant eigenvalues that can be obtained from evaluating the Jacobian of the $n-1$ species system at $\breve{\mathbf{P}}$. From equation [S44], it is clear that the remaining relevant eigenvalue is directly given by $\frac{\partial}{\partial P_{n}}\left(\frac{\mathrm{d} P_{n}}{\mathrm{dt}}\right)_{\mathbf{P}=\widetilde{\mathbf{P}}}$ and that linear independence of the associated eigenvector is ensured by $v_{n n} \neq 0$. We can rewrite this remaining relevant eigenvalue as:

$\lambda_{n} \equiv \frac{\partial}{\partial P_{n}}\left(\frac{\mathrm{d} P_{n}}{\mathrm{dt}}\right)_{\mathbf{P}=\widetilde{\mathbf{P}}}=\frac{\operatorname{det} \mathbf{A}_{n}}{-\sum_{j=1}^{n-1} \operatorname{det} \breve{\mathbf{A}}_{j}}=-\frac{\operatorname{det} \mathbf{A}_{n}}{\psi_{C}^{(n-1)}}$

In which $\psi_{C}^{(n-1)}$ indicates the sum of feedback effects experienced within the community of $n$ 1 species (i.e. all species except species $n$ ). As the derivation of equation [S45] from equation [S40] is somewhat lengthy, we will leave this derivation for the end of this section.

From the result expressed in equation [S45], we can make a number of observations. First, as $\lambda_{n}$ is the only eigenvalue that can be influenced by $P_{n}$, this is the relevant eigenvalue to assess the invasion (recovery) potential of $P_{n} . P_{n}$ is able to invade (recover) when $\lambda_{n}>0$. Second, stable coexistence of the community of $n$ species not only requires that $P_{n}$ invades (recovers when rare), but from the feasibility condition (equation [S35]) it follows that also the sum of feedback effects experienced in the $n$ species community, $\psi_{C}^{(n)}$, needs to be of the same sign as any $\operatorname{det} \mathbf{A}_{j}$, including $\operatorname{det} \mathbf{A}_{n}$. This result implies that for a stably coexisting community of $n$ species, the sign of $\psi_{C}^{(n)}$ needs to be opposite to the sum of feedback effects experienced in a community of $n-1$ species (i.e. $\psi_{C}^{(n-1)}$ ). We have previously shown that stable coexistence for the two-species case requires $\psi_{C}^{(2)}<0$ (equation [S17]; Fig. S1). This means 
that stable coexistence for a three-species community requires $\psi_{C}^{(3)}>0$ (as $\lambda_{i}$ in equation [S45] needs to be positive for species $n$ when invading the $n-1$ species community). This notion can be generalized into stability requiring $\psi_{C}^{(n)}<0$ when the community contains an even number of species, and $\psi_{C}^{(n)}>0$ when this species number is odd. This observation can be understood from the more general observation that the determinant of the Jacobian of a stable system with an even number of state variables is positive, and the determinant of the Jacobian of a stable system with an odd number of state variables is negative (e.g. $\left.{ }^{47}\right)$. The above results lend themselves well for interpretation in terms of ecological feedback mechanisms, through application of the analysis methods proposed by Levins (e.g. in ${ }^{24}$ ). Levins defined the feedback generated by a $\mathrm{k}^{\text {th }}$ order Jacobian matrix, $J_{k}$, using its determinant $D^{(k)}$ :

$J_{k}=(-1)^{k+1} D^{(k)}$

For the current system, we can continue from equation [S43] to write the determinant of the Jacobian of an $n$-species system as:

$D^{(n)}=\frac{\partial F_{n}}{\partial P_{n}} \operatorname{det}[\breve{J}]$

Using equations [S33] and [S45], we can rewrite the right-hand side of equation [S47] as:

$\frac{\partial F_{n}}{\partial P_{n}} \operatorname{det}[\breve{J}]=-\frac{\operatorname{det} \mathbf{A}_{n}}{\psi_{C}^{(n-1)}} \operatorname{det}[\breve{J}]=-\frac{\breve{\hat{w}} \operatorname{det}[\breve{J}]}{\operatorname{det} \breve{\mathbf{A}}} \operatorname{det} \mathbf{A}_{n}$

Equation [S48] shows that the Jacobian for a system in which an $n^{\text {th }}$ species is invading (or rare) can be written as a product of two feedback factors, the first factor referring to the feedbacks within the $n-1$ species community, and the second factor quantifying the effect of these $n-1$ species on the invading (or rare) species $n$. Provided that the $n-1$ species system is 
feasible and stable (meaning that the first factor generates negative feedback), the second factor will determine whether species $n$ can invade or not. If both terms generate negative feedback in the $n$ species system, the total feedback in the system is negative ${ }^{24}$, and species $n$ will be able to invade (or recover when rare). We note that the minus sign in equation [S48] appears because the Jacobian is evaluated at $\mathbf{P}=\widetilde{\mathbf{P}}$, meaning that positive feedback on perturbations at this point (which are larger values of $P_{n}$ than its equilibrium value of zero) imply persistence of species $n$. When considering the coexistence equilibrium, these perturbations would be in the opposite direction (i.e. smaller values of $P_{n}$ than at equilibrium), and thus the sign of the feedback would change as well. In other words, positive feedback in the invasion condition contributes to negative feedback in the coexistence condition (see e.g. Fig. S1b, but see also the comments regarding the sufficiency of the $I_{C}$ criterion below). We can therefore apply Levins' theorem to the second factor in equation [S48] to assess the contribution of this factor (the species-specific feedback for species $n$ ) to the community-level feedback in the $n$-species system. Recalling that $\operatorname{det} \mathbf{A}_{j}$ is a determinant of the order $n$-1 (i.e. $\sigma^{n-1}$ ), we can thus use equation [S46] to calculate the contribution of each sum of species-specific feedback effects to the community-level feedback ${ }^{24}$ :

$I_{j}=(-1)^{n} \operatorname{det} \mathbf{A}_{j}$

As noted above, fulfilling the feasibility condition (equation [S35]) ensures that all $\operatorname{det} \mathbf{A}_{i}$, and thus all $I_{j}$, carry the same sign. This means that we can now use equation [S49] to obtain a generalized community-level feedback coefficient:

$$
I_{C}=\sum_{j=1}^{n} I_{j}=\sum_{j=1}^{n}(-1)^{n} \operatorname{det} \mathbf{A}_{j}=(-1)^{n} \sum_{j=1}^{n} \operatorname{det} \mathbf{A}_{j}
$$


From equation [S50] it follows that negative community-level feedback is generated in evennumbered communities with negative $\psi_{C}^{(n)}$ (i.e. $\sum_{j=1}^{n} \operatorname{det} \mathbf{A}_{j}$ ) and negative feedback is generated in odd-numbered communities with positive $\psi_{C}^{(n)}$. It is thus clear that $I_{C}$ encompasses the invasibility criteria of each of the $n$ species within the community, with the feasibility condition ensuring sign uniformity of these criteria.

A third observation from equation [S45] is that the relevant eigenvalue for species recovery is defined by a term of order $\sigma^{n-1}$ in the numerator and a term of order $\sigma^{n-2}$ in the denominator. Hence, independently of the community size, the sign of the relevant eigenvalue is determined by an expression consisting of first order terms. This observation provides a possible explanation for the success of explaining community diversity patterns in previous approaches through the use of the averaged pair-wise interaction coefficients as predictors for abundance patterns ${ }^{10,11,23,33}$.

Finally, we note that equation [S45] describes the eigenvalue that is associated with the eigenvector that is perpendicular to the $n-1$ dimensional state space obeying $\sum_{i=1}^{n-1} P_{i}=0$. This means that the same argument can be applied to derive the irrelevant eigenvector for the $n$ species system. Specifically, for an $n$-species system, its irrelevant eigenvalue is described by:

$\lambda_{i r r}=-\frac{\operatorname{det} \mathbf{A}}{\psi_{C}^{(n)}}=-\hat{w}$

From equation [S51] it follows that for feasible equilibrium points (i.e. obeying equation [S35]), the irrelevant eigenvalue always consists of (only) a real negative part. 
Is the community interaction coefficient a sufficient criterion for species coexistence?

So far, we have established that the sign of $I_{C}$ provides a necessary condition for plant community coexistence. Next, we can turn to the question whether the $I_{C}$ criterion is also a sufficient condition for stable coexistence. In fact, $I_{C}$ is not a sufficient condition, which can be explained by the following two observations.

First, local stability of the community coexistence equilibrium depends on the eigenvalues of the Jacobian matrix, evaluated at this equilibrium point. Although the sign structure of the Jacobian matrix has proven to be a very powerful tool in analysis of community matrices (examples presented in $^{53,54}$ ), in the model described here we can find for the same sign structure (e.g. all entries of the Jacobian being negative) of the Jacobian both positive and negative dominant eigenvalues. This example illustrates that there is no straightforward link between sign changes in the Jacobian, the resulting eigenvalues, and the role of $I_{C}$ in governing these sign changes. This means that even if the $I_{C}$ criterion is fulfilled, and all species are able to recover when rare, the community coexistence equilibrium may not be an attractor. In this case, we can find a positive dominant eigenvalue, yet all species persist, by means of periodic oscillations, which can be understood from the competitive network becoming (partly) intransitive (see Supplementary Information section 4). This result shows that the $I_{C}$ criterion is not a sufficient condition for stable coexistence, but it is a necessary condition for species persistence. In contrast, a negative real part of the leading eigenvalue associated with the community coexistence equilibrium is not a necessary condition for species persistence.

Second, the above analysis has started from the premise that a species $P_{n}$ invades a community of $n-1$ species that are stably coexisting. Of course, it is conceivable that this 
latter community equilibrium is unstable. In this case, a positive $\lambda_{n}$ could be obtained from equation [S45], but for the wrong reasons. In this case, the invasion condition is ill-posed, as it is not considering the equilibrium that $P_{n}$ actually needs to invade. This other equilibrium (with less than $n-1$ species) would need to be analysed instead. If it turns out that the community equilibrium consisting of $n-1$ species is unstable, this leaves the possibility of the system being attracted by one of the $2^{\mathrm{n}-2}-1$ other non-trivial equilibria, which becomes a demanding task to examine. Similarly, even if species $n$ is able to invade a community equilibrium, this does not preclude the possibility of the existence of alternate stable equilibria that cannot be invaded by species $n$. Due to these two reasons, the $I_{C}$ criterion is a necessary, but not a sufficient condition for stable community coexistence.

The characteristics of the coexistence equilibrium as described above can be attributed to the fact that the interaction matrices A considered are asymmetric. When symmetric interaction matrices $^{7}$ or anti-symmetric matrices ${ }^{5}$ are considered, coexistence equilibria are globally stable, meaning that the system will always be drawn toward the equilibrium point regardless of the species' initial frequencies. Symmetric interaction matrices emerge when interactions are based on processes such as niche overlap ${ }^{7}$, whereas anti-symmetric matrices describe interactions in which there is a winner and a $\operatorname{loser}^{5}$. When studying frequency-dependent interactions such as those originating from plant-soil feedback, however, asymmetry of interactions is frequently observed ${ }^{21,55-58}$. Interestingly, despite these differences between asymmetric and (anti)-symmetric interaction matrices, we did find for all types of interaction matrices that there can be a strong correlation between average pair-wise feedback and (rescaled) community-level feedback (Fig. S2, a result shown for asymmetric interaction matrices in the main text of the manuscript). 


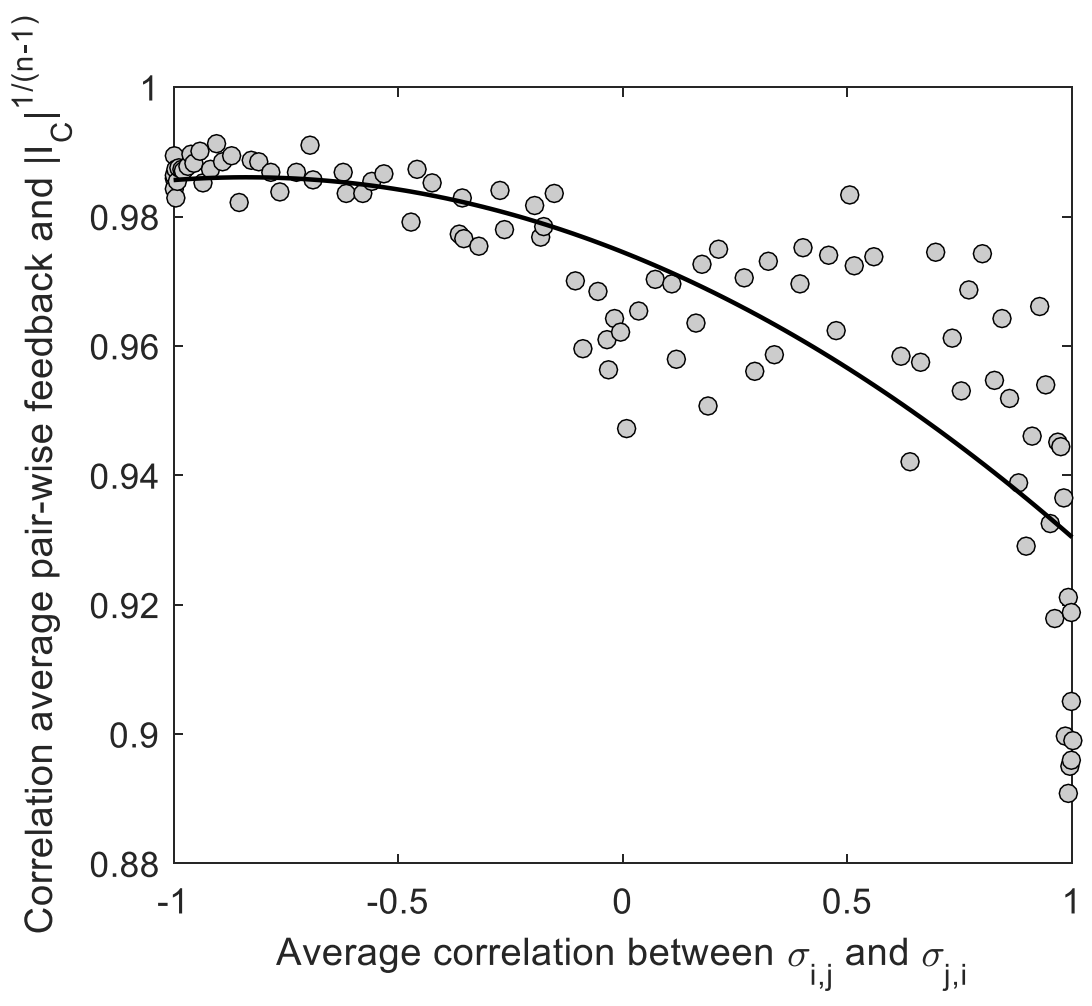

\section{Supplementary Figure S2 | Average pair-wise feedback correlates with community-level feedback for} anti-symmetric, asymmetric and symmetric interaction matrices. Data points show the (Pearson) correlation between average pair-wise feedback and (rescaled) community-level feedback $I_{C}$ for a set of 50 simulations that span a feedback gradient. The number of species in the community was chosen to be odd $(n=11)$, to enable persistence of communities characterized by anti-symmetric interaction matrices ${ }^{5}$. For all simulations, conspecific coefficients were randomly varied between $0.1-\left(4+\Delta_{\mathrm{i}}\right) s$ and $0.2-\left(4+\Delta_{\mathrm{i}}\right) s$, in which $s$ is a parameter determining the average difference between conspecific and heterospecific effects and the variation within heterospecific effects, and $\Delta_{\mathrm{i}}$ is a parameter determining the strength of the feedback gradient in the $i^{\text {th }}$ simulation of the series. This latter parameter was varied between $\Delta_{1}=\Delta_{\max } / 50$ and $\Delta_{50}=\Delta_{\max }$ for each set of simulations. The figure was created using $s=0.16$ and $\Delta_{\max }=0.25$. For each simulation, the upper triangular part of the matrix was filled with heterospecific coefficients (indicated with $\sigma_{i j}$ ) that were randomly drawn from the uniform distribution $X_{i}=\mathrm{U}\left(-\left(1+\Delta_{\mathrm{i}}\right) s,\left(1+\Delta_{\mathrm{i}}\right) s\right)$. For each of these sets, the pair-wise correlation between $\sigma_{i j}$ and their lower triangular counterparts $\sigma_{j i}$ was varied by using the following distribution for $\sigma_{j i}$ :

$Y_{\mathrm{i}}=\varphi X_{i}+(1-|\varphi|) * \mathrm{U}\left(-\left(1+\Delta_{\mathrm{i}}\right) s,\left(1+\Delta_{\mathrm{i}}\right) s\right)$. Here, $\varphi$ determines the correlation between $\sigma_{i j}$ and $\sigma_{j i}$. Specifically, $\varphi=-1$ creates antisymmetric interaction matrices, $\varphi=1$ creates symmetric interaction matrices and $\varphi=0$ creates asymmetric interaction matrices. In this figure, $\varphi$ was varied between -1 and 1 , with increments of 0.02 . 
The above constraints on the applicability the $I_{C}$ criterion can be circumvented in scenarios of community assembly. With stepwise addition of species, one can ascertain that the invasion condition is properly described by equation [S45], and that feedback within the resident community is properly described by the first factor on the right hand side of equation [S48]. However, even in an assembly scenario, it is possible that all species persist, but that they form a (partly) intransitive network leading to species oscillations (see Supplementary Information section 4). In this case, invasion success of a new species may be time-dependent (i.e. at which point in the cycle it attempts to invade), which is not captured by equation [S45].

\section{Derivation of equation [S45] from equation [S40]}

We start the derivation by specifying equation [S40] for the invading species $n$ :

$$
\frac{\mathrm{d} P_{n}}{\mathrm{dt}}=P_{n}\left(\sum_{j=1}^{n} \sigma_{n j} P_{j}-\sum_{j=1}^{n} \sum_{k=1}^{n} \sigma_{j k} P_{j} P_{k}\right)
$$

Substituting equation [S52] into the definition of $\lambda_{n}$ (equation [S45]), and calculating the derivative with respect to $P_{n}$, we obtain:

$$
\lambda_{n}=\frac{\partial}{\partial P_{n}}\left(\frac{d P_{n}}{d t}\right)=\left(\sum_{j=1}^{n} \sigma_{n j} P_{j}-\sum_{j=1}^{n} \sum_{k=1}^{n} \sigma_{j k} P_{j} P_{k}\right)+P_{n} \frac{\partial}{\partial P_{n}}\left(\sum_{j=1}^{n} \sigma_{n j} P_{j}-\sum_{j=1}^{n} \sum_{k=1}^{n} \sigma_{j k} P_{j} P_{k}\right)
$$

Substituting $\mathbf{P}=\widetilde{\mathbf{P}}$, and noting that $P_{n}=0$ (i.e. the invasion condition), we can write:

$$
\lambda_{n}=\left(\sum_{j=1}^{n-1} \sigma_{n j} \breve{P}_{j}-\sum_{j=1}^{n-1} \sum_{k=1}^{n-1} \sigma_{j k} \breve{P}_{j} \breve{P}_{k}\right)
$$

Now we can specify equation [S54] for the case in which the $n-1$ species system is in equilibrium (see equation [S31]): 


$$
\breve{P}_{i}=\frac{\operatorname{det} \breve{\mathbf{A}}_{i}}{\sum_{j=1}^{n-1} \operatorname{det} \breve{\mathbf{A}}_{j}}=\breve{w} \frac{\operatorname{det} \breve{\mathbf{A}}_{i}}{\operatorname{det} \breve{\mathbf{A}}^{-1}}
$$

From equations [S28], [S31] and [S33], we know that:

$$
\breve{w} \sum_{k=1}^{n-1} \operatorname{det} \breve{\mathbf{A}}_{k}=\sum_{l=1}^{n-1} \sigma_{j l} \operatorname{det} \breve{\mathbf{A}}_{l}=\operatorname{det} \breve{\mathbf{A}} \text { for any } j=1, \ldots, n-1
$$

Which means that equation [S54] for the specific case can be written as:

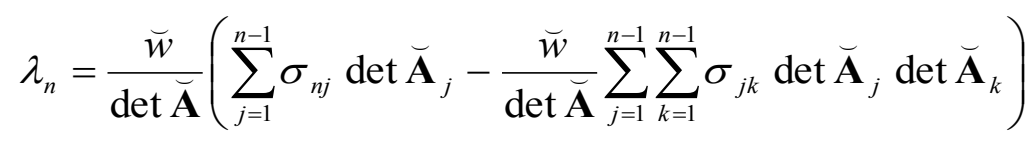

Using the definition of $\breve{w}$, we can write:

$$
\lambda_{n}=\frac{\breve{w}}{\operatorname{det} \breve{\mathbf{A}}}\left(\sum_{j=1}^{n-1} \sigma_{n j} \operatorname{det} \breve{\mathbf{A}}_{j}-\frac{\breve{w}}{\operatorname{det} \breve{\mathbf{A}}_{j=1}^{n-1}} \sum^{\operatorname{det}} \breve{\mathbf{A}}_{j} \operatorname{det} \check{\mathbf{A}}\right)
$$

Which is equal to:

$$
\lambda_{n}=\frac{1}{\sum_{l=1}^{n-1} \operatorname{det} \breve{\mathbf{A}}_{l}}\left(\sum_{j=1}^{n-1} \sigma_{n j} \operatorname{det} \breve{\mathbf{A}}_{j}-\operatorname{det} \breve{\mathbf{A}}\right)
$$

We now introduce the matrix $\mathbf{A}_{n}$, a matrix equal to the interaction matrix of the $n$ species system of $n$ species except for the last $\left(n^{\text {th }}\right)$ column, which is replaced by a column vector of ones. We rewrite the determinant of this matrix using the Laplace expansion:

$$
\operatorname{det} \mathbf{A}_{n}=\sum_{l=1}^{n}(-1)^{n+l} \sigma_{n l} \operatorname{det} \mathbf{M}_{n l}=\sum_{l=1}^{n-1}(-1)^{n+l} \sigma_{n l} \operatorname{det} \mathbf{M}_{n l}+(-1)^{2 n} \operatorname{det} \breve{\mathbf{A}}
$$

where $\mathbf{M}_{n l}$ is defined as the minor matrix of $\mathbf{A}$ obtained when removing the $n^{\text {th }}$ row and the $l^{\text {th }}$ column from matrix $\mathbf{A}_{n}$. To derive equation [S59], we also used the fact that $\mathbf{M}_{n n}=\breve{\mathbf{A}}$. We now observe that each matrix $\mathbf{M}_{n l}$, for $l<n$, has a last column of ones (as in $\mathbf{A}_{n}$ ) and thus can be 
obtained from $\breve{\mathbf{A}}_{l}$ switching columns $n$-l-1 times. Their determinants are therefore related as $\operatorname{det} \mathbf{M}_{n l}=(-1)^{n-l-1} \operatorname{det} \breve{\mathbf{A}}_{l}$, and thus substituting this into expression for $\operatorname{det} \mathbf{A}_{n}$ we obtain:

$\operatorname{det} \mathbf{A}_{n}=\sum_{l=1}^{n}(-1)^{2 n-1} \sigma_{n l} \operatorname{det} \breve{\mathbf{A}}_{l}+\operatorname{det} \breve{\mathbf{A}}=-\sum_{l=1}^{n} \sigma_{n l} \operatorname{det} \breve{\mathbf{A}}_{l}+\operatorname{det} \breve{\mathbf{A}}^{.}$

Using this equality, we can obtain from equation [S60]:

$\lambda_{n}=\frac{1}{\sum_{l=1}^{n-1} \operatorname{det} \breve{\mathbf{A}}_{l}}\left(\operatorname{det} \breve{\mathbf{A}}-\operatorname{det} \mathbf{A}_{n}-\operatorname{det} \breve{\mathbf{A}}\right)=\frac{-\operatorname{det} \mathbf{A}_{n}}{\sum_{l=1}^{n-1} \operatorname{det} \breve{\mathbf{A}}_{l}}$

Which is equivalent to equation [S45]. 


\section{Species persistence despite instability of the coexistence equilibrium}

We found that negative community-level feedback $\left(I_{C}<0\right)$ does not require that the real part of the leading eigenvalue of the coexistence equilibrium is negative. This means that even when the coexistence equilibrium is unstable to perturbations, negative community-level feedback may still be present, and it may enable the persistence of all species. This result shows an important difference between $I_{C}$ (which is derived from invasion conditions) and more traditional approaches focusing on local stability analysis of the coexistence equilibrium. Here we show that in these cases, the resulting species dynamics are described by temporal oscillations in species frequencies, leading to cyclic succession ${ }^{22,26}$. Figure S3 shows an example of a ten-species system where oscillations are sustained by negative community-level feedback. This example was obtained through generation of interaction matrices for ten species, choosing coefficients from a uniform distribution ranging from 0.1 to 0.2 (conspecific), and from 0.1 to 0.9 (heterospecific), following ${ }^{10,23}$. Temporal oscillations maintained through negative community-level feedback requires: 1) A feasible coexistence equilibrium 2) The real part of the leading eigenvalue of this coexistence equilibrium being positive 3) The community interaction coefficient, $I_{C}$, being negative. It is important to note that $I_{C}<0$ is a necessary, but not a sufficient condition for species persistence (see Supplementary Information section 3). Therefore, for an interaction network fulfilling the above criteria, occurrence of temporal oscillations and species persistence was investigated using numerical model runs (Runge-Kutta $4^{\text {th }}$ and $5^{\text {th }}$ order integration as implemented in Matlab v. 7.12).

It should also be noted that in real systems, negative community-level feedback ensuring species persistence could occur in communities that do not exhibit the regular oscillations in 
species frequencies with relatively large amplitude as shown in Figure S2, as many external (spatial and temporal) factors could dampen, stabilize or desynchronize such internal dynamics 22,59 .

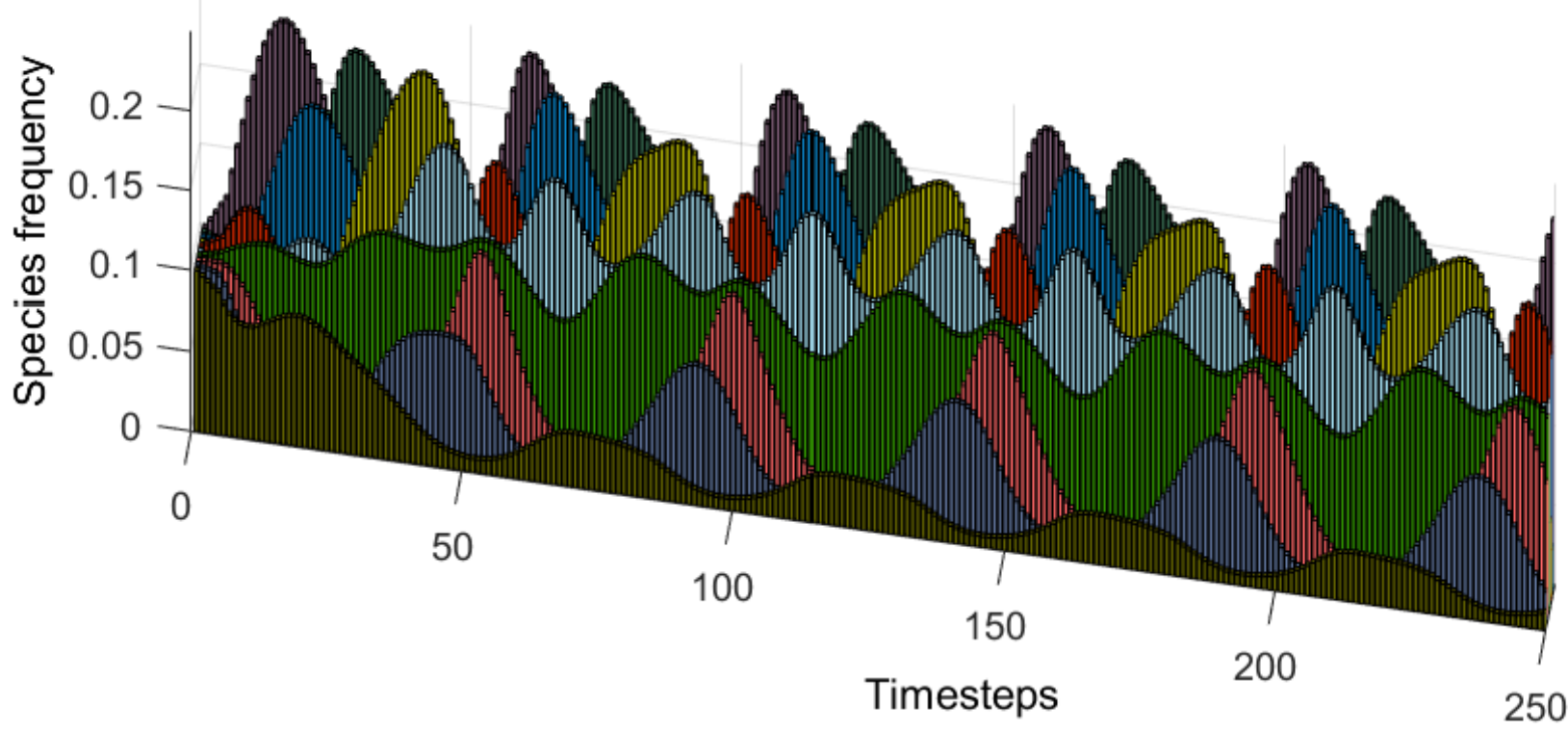

Supplementary Figure S2 | Example of a plant community where ten species (indicated with different colours) persist by means of temporal oscillations that are driven by negative feedback. The coexistence equilibrium is feasible, yet unstable to perturbations. In this community, however, the community interaction coefficient, $I_{C}$, is negative, which enables each species to recover when rare (provided that all other species of the community are also still present in non-zero densities). Conspecific frequency-dependent effects varied between 0.1 and 0.2 , heterospecific frequency-dependent effects varied between 0.1 and 0.9 ; more specifically, the matrix used in this figure is given in the text (equation [S62]).

The oscillations as shown in Fig. S3 are driven by (partly) intransitive dynamics, and the occurrence of such dynamics is highly dependent on the specific structure of the interaction matrix. In the model framework, fitness of each species (governed by $w_{i}$ in equation [S10]) is a dynamic property that depends on the current frequencies of all species. As frequencies 
change over time, species' fitnesses change over time as well. Considering the interaction matrix A used to generate the dynamics shown in Fig. S3, it is insightful to consider the heterospecific effects that a species $i$ exerts on all the species within the community. These effects are given by all the off-diagonal elements within the $i^{\text {th }}$ column of the interaction matrix:

$$
A=\left[\begin{array}{cccccccccc}
0.18 & 0.8 & 0.2 & \mathbf{0 . 9} & 0.6 & 0.2 & 0.2 & 0.6 & 0.8 & 0.4 \\
\mathbf{0 . 8} & 0.16 & 0.8 & 0.4 & 0.4 & 0.8 & 0.7 & 0.3 & 0.6 & 0.3 \\
0.7 & 0.6 & 0.14 & 0.1 & 0.5 & 0.8 & 0.6 & 0.3 & 0.2 & 0.3 \\
0.2 & 0.1 & 0.3 & 0.17 & 0.3 & 0.7 & \mathbf{0 . 9} & 0.3 & \mathbf{0 . 8} & \mathbf{0 . 8} \\
0.3 & 0.8 & 0.1 & \mathbf{0 . 9} & 0.18 & 0.3 & 0.5 & 0.6 & 0.1 & 0.7 \\
\mathbf{0 . 8} & 0.5 & 0.7 & 0.1 & 0.6 & 0.19 & 0.4 & 0.5 & 0.4 & 0.6 \\
0.4 & \mathbf{0 . 8} & 0.5 & 0.1 & 0.3 & 0.8 & 0.11 & 0.6 & 0.6 & 0.4 \\
0.1 & 0.8 & 0.2 & 0.4 & \mathbf{0 . 8} & 0.8 & 0.6 & 0.16 & 0.5 & 0.1 \\
0.7 & 0.2 & 0.5 & 0.4 & 0.7 & \mathbf{0 . 9} & 0.5 & 0.3 & 0.1 & 0.4 \\
0.2 & 0.6 & 0.7 & 0.3 & 0.5 & 0.3 & 0.8 & \mathbf{0 . 8} & 0.2 & 0.14
\end{array}\right]
$$

These within-column differences in heterospecific effects and the conspecific effect indicate the relative fitness differences between competitors in an environment dominated by species $i$. The highest values indicate the most fit species, as these species receive the least negative effects from species $i$. The assymmetrical nature of matrix $\mathbf{A}$ means that for a given species pair, it is possible that only one of the species is able to do well in the other speciesdominated environment. In these cases, we would expect a unidirectional succession trajectory, in which one species is able to overtake an environment dominated by the other species, but the reverse transition does not take place. Intransitive dynamics can then occur if consecutive transitions eventually create an environment in which the original species can again do well ${ }^{26,60}$. Focusing on the maximum (non-reciprocal) heterospecific coefficients in each column on the right hand side of equation [S62], we can identify three of these intransitive loops: loop $1: 1 \rightarrow 2 \rightarrow 7 \rightarrow 4 \rightarrow 1$, loop $2: 4 \rightarrow 5 \rightarrow 8 \rightarrow 10 \rightarrow 4$, and 
loop 3: $1 \rightarrow 6 \rightarrow 9 \rightarrow 1$. In the interaction matrix $\mathbf{A}$, there are additional maximum heterospecific coefficients (for species 1,5 and 8 in an environment dominated by species 2, for example), but these possible transitions do not likely lead to intransitive loops involving more than 2 species given the remaining matrix structure. Interestingly, none of these loops lead to sustained oscillations when considering the sub-systems only including these species; loops 2 and 3 lead to a stable equilibrium, whereas loop 1 is not feasible, as species 4 will be excluded. However, because of the connections between these loops within the 10 -species system (Fig. S4a), species 1 and 10 alternate in frequency and species 4 peaks in frequency when $10 \rightarrow(4 \rightarrow) 1$ (Fig. S4b). It should be noted that the intransitive dynamics in this 10 species system emerge even though most pair-wise interactions are characterized by negative feedback, i.e. $I_{S}<0$ and there is mutual invasibility. Some cases that could contribute to strict intransitivity occur, for example species 8 would be excluded when competing solely with species 1 or species 10 . Although effects of such strong competitors are clearly visible in the weaker species' dynamics (Fig. S4b), these strict interactions are not crucial for generating sustained oscillations. More specifically, in the example shown here, none of the effects $\sigma_{i j}<\sigma_{i i}$ is crucial for maintaining a positive real part of the leading eigenvalue while $I_{C}<0$.

This observation corroborates with the above notion that the heterospecific effects determine relative fitness in single-species-environments of the remaining competitors. This creates a dynamic hierarchy and, depending on the specific structure of the matrix $\mathbf{A}$, the possibility of intransitive dynamics. 

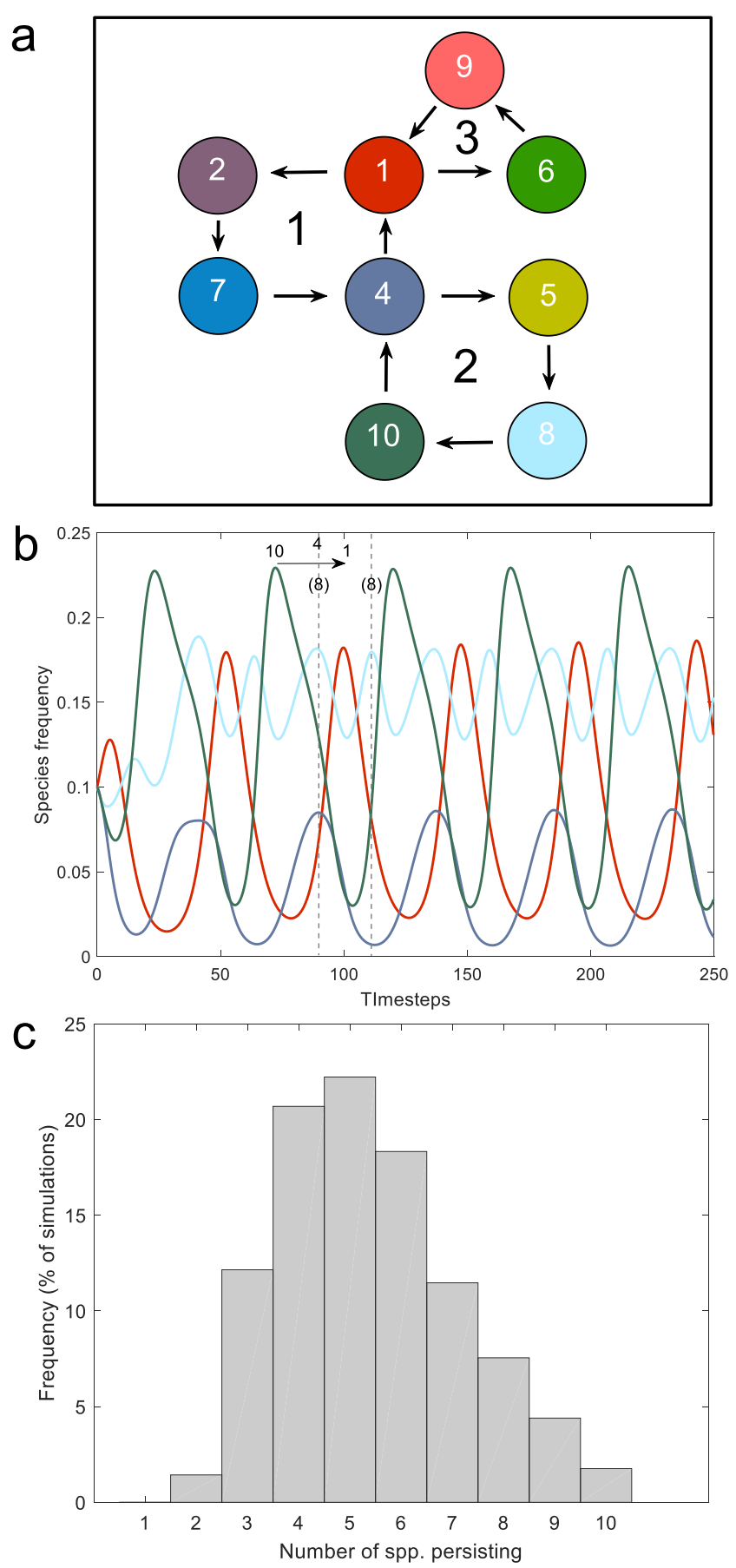

\section{Supplementary Figure $\quad$ S4 $\quad$ Temporal} oscillations as shown in figure $\mathrm{S} 2$ emerge from intransitive loops of alternating species fitness.

a) Fitness in single-species dominated environments (equation [S62]) points to three intransitive loops for the 10-species system. Species are represented by the circles, while the loops are indicated by the numbers between them. (Species 3 was not crucial in generating oscillations here, and has therefore been omitted). b) Temporal oscillations of species 1, 4, 8 and 10 (Species are color-coded as in panel a) and Fig. S2. Species 4 can persist due to increased fitness during the transition $10 \rightarrow 1$ (i.e. between dominance of 10 to dominance of 1), connecting loops 1 and 2. The dynamics of species 8 show the strong control of superior competitors, but such strict hierarchies are not necessary to drive sustained oscillations. c) Histogram showing the number of species persisting in 10,000 runs of this 10-species system, but with the heterospecific feedback coefficients being randomized within each species (i.e. row in the interaction matrix). The large range of persisting species shows the potential importance of intransitivity. Full-factorial experimental designs will be needed in future studies to accurately describe and model such networks. 
To further illustrate this latter point, we performed additional model runs in which we randomized the heterospecific effects within each plant species (i.e. within each row of the interaction matrix A, equation [S62]). We note that this randomization does not affect the average heterospecific feedback experienced by each species, nor does it affect the average pair-wise feedback strength within the system. However, a sample of 10,000 randomizations yielded a large range in the number of species persisting in the system (Fig. S4c; 2-10, mean number of persisting species: 5.4). These results stress the importance of quantifying all species interactions within a network. Many current databases, including the dataset extracted from the FIA surveys and used in this study (see Supplementary Information section 5), lack information on all heterospecific effects, and only contain (row-) averaged heterospecific effects $^{11,31}$. In this study, we addressed this lack of information by analyzing community diversity conservatively in our community assembly procedure, by only considering systems stable if the real part of the leading eigenvalue was negative. Future studies using fullfactorial designs may shed more light on the importance of intransitive dynamics maintaining community diversity (see also Supplementary Information section 7), and using randomization tests as used above (which have also been used in food web studies ${ }^{61}$ ) can shed light on the sensitivity of such communities to perturbations (such as losses or introductions of new species).

In all cases in which persistent oscillations were encountered, the eigenvalues with positive real parts were complex. Previous studies revealed that for systems involving four or more species, it is also possible that oscillations become chaotic ${ }^{14,62-64}$. For example, Schnabl et al. ${ }^{63}$ provided a full characterization of the strange attractor in a four-species replicator model. More specifically, they investigated a family of interaction matrices: 


$$
\mathbf{A}=\left[\begin{array}{cccc}
0 & 0.5-0.437 v & -0.1-0.1 v & 0.1+0.337 v \\
1.1-0.563 v & 0 & -0.6+0.564 v & -0.001 v \\
-0.5-0.035 v & 1-0.62 v & 0 & 0.655 v \\
1.7+\mu-1.164 v & -1-\mu+0.968 v & -0.2+0.196 v & 0
\end{array}\right]
$$

In which $\mu$ and $v$ are parameters. Here we verified that for the entire $(\mu, v)$ parameter space examined in $^{63}(-1 \leq \mu \leq 1.2$ and $-1.5 \leq v \leq 0.5)$, the condition $I_{C}<0$ applies $\left(-9.94 \leq I_{C} \leq-\right.$ 0.086). Notably, this $(\mu, v)$ parameter space encompasses a subset of conditions in which chaotic dynamics are encountered ${ }^{63}$.

In contrast, a two-species system (as introduced in Supplementary Information section 2) cannot exhibit any persistent oscillatory behaviour, as its eigenvalues are described by:

$$
\lambda_{1}=-\frac{\sigma_{11} \sigma_{12}-2 \sigma_{12} \sigma_{21}+\sigma_{21} \sigma_{22}}{2 I_{S}}-\sqrt{\frac{\left(\sigma_{11} \sigma_{12}-2 \sigma_{11} \sigma_{22}+\sigma_{21} \sigma_{22}\right)^{2}}{4 I_{S}^{2}}}
$$

And:

$$
\lambda_{2}=-\frac{\sigma_{11} \sigma_{12}-2 \sigma_{12} \sigma_{21}+\sigma_{21} \sigma_{22}}{2 I_{S}}+\sqrt{\frac{\left(\sigma_{11} \sigma_{12}-2 \sigma_{11} \sigma_{22}+\sigma_{21} \sigma_{22}\right)^{2}}{4 I_{S}^{2}}}
$$

Here, equation [S64a] is the system's relevant eigenvalue, which can be rewritten as:

$$
\lambda_{1}=I_{S} \hat{P}_{1} \hat{P}_{2}
$$

This is a useful result, as it shows that the relevant eigenvalue can only consist of a real positive part when the Jacobian is evaluated at a feasible equilibrium point. Importantly, perturbations of the system along the associated eigenvector, due to the lack of an imaginary part, will form a so-called straight-line solution (e.g. $\left.{ }^{65}\right)$ of the form:

$$
\mathbf{P}(t)-\hat{\mathbf{P}}=\sum_{i=1}^{2} L_{1} e^{\lambda_{1}\left(t-t_{0}\right)} v_{1, i}
$$


In which $v_{1, i}$ are the components of the eigenvector associated with $\lambda_{1}$, and $L_{1}$ is the magnitude of the initial perturbation along this eigenvector component. Because $\left|v_{1,1}\right|+\left|v_{1,2}\right|>0$, while $v_{1,1}+v_{1,2}=0$ (as noted in Supplementary Information section 3), it necessarily follows that $v_{1,1}=-v_{1,2} \neq 0$. This means that the straight-line solution (equation [S66]) will intersect with an endpoint of the line segment $P_{1}+P_{2}=1$, at which point one species has been driven to exclusion. Although the analytical expression for the irrelevant eigenvalue for the twospecies system can be rewritten into the form that holds for communities of any number of species (equation [S51] in Supplementary Information section 3), the relevant eigenvalues for communities of more than two species can no longer be described as conveniently as equation [S65]. Therefore, we tested numerically whether the above conclusions for the twospecies system held for larger communities (simulations were performed for four-species and ten-species communities, respectively). Specifically, these numerical simulations agreed with the inferences made for the two-species system, in that: 1) All coexistence equilibrium points in systems with $I_{C}>0$ have at least one relevant eigenvalue that only contains a positive real part (Fig. S5a,c). 2) Perturbations along the eigenvectors that are associated with these eigenvalues continue to grow until at least one species has gone extinct (Fig. S5b,d). These results suggest that $I_{C}>0$ excludes the possibility of the coexistence equilibrium functioning as any type of attractor that enables persistence of all community members. 

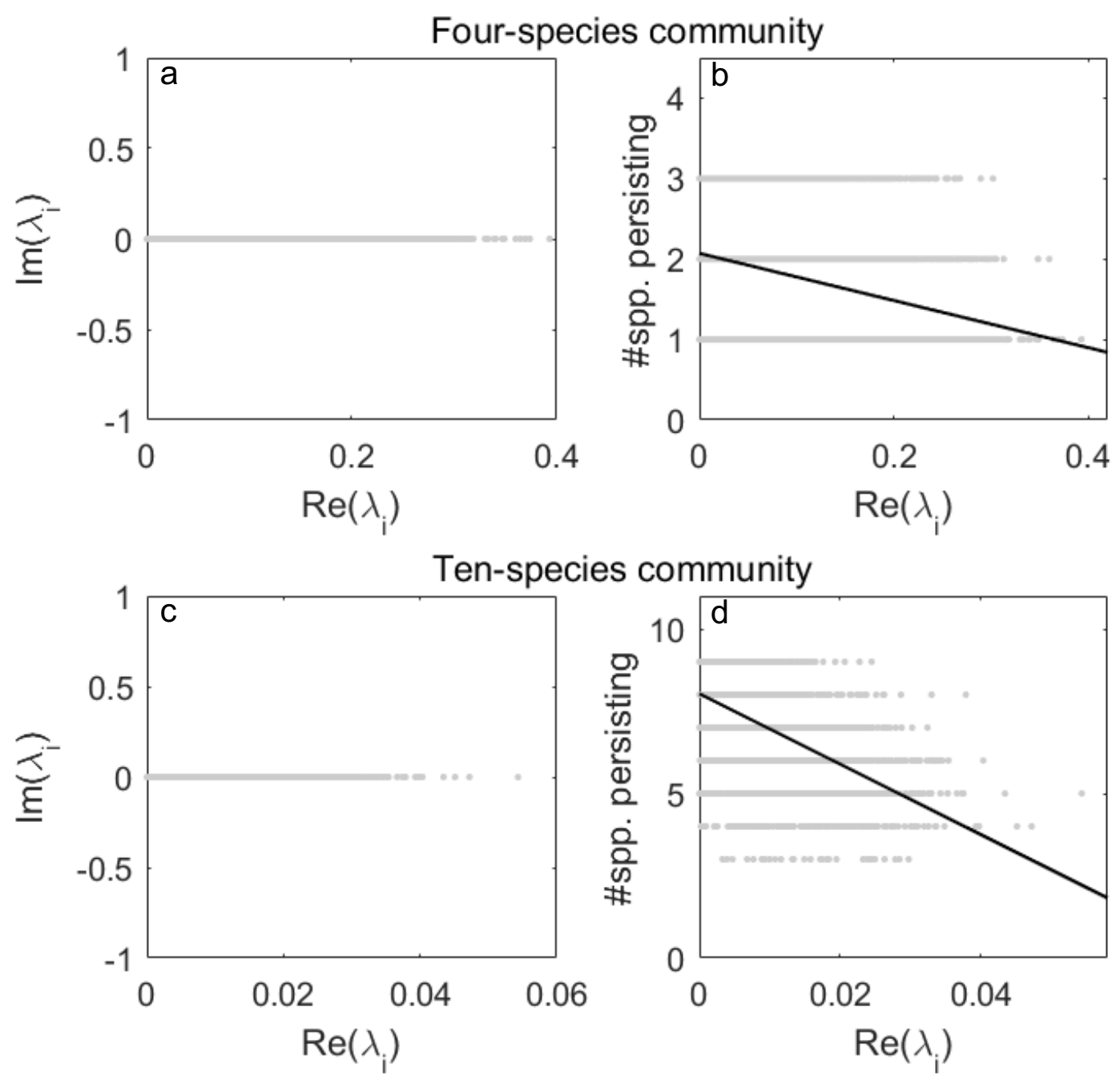

Supplementary Figure S5 | Perturbations of multi-species communities characterized by $I_{C}>0$ lead to species exclusion. a) A set of 10,000 four-species communities characterized by $I_{C}>0$ contain at least one eigenvalue that only contain a positive real part. b) Perturbations of the unstable coexistence equilibrium along the straight-line solution described by this positive eigenvalue and its associated eigenvector (see equation [S66] in the text) leads to exclusion of at least one species. The number of species exclusions increased with the magnitude of the eigenvalue. Filled line shows a linear fit: $y=-2.93 x+2.07, R_{a d j}{ }^{2}=0.05, p<1.10^{-100}$. c) A set of 10,000 ten-species communities characterized by $I_{C}>0$ contain at least one eigenvalue that only contain a positive real part. d) Perturbations of the unstable coexistence equilibrium along the straight-line solution described by this positive eigenvalue and its associated eigenvector leads to exclusion of at least one species. The number of species exclusions increased with the magnitude of the eigenvalue. Filled line shows a linear fit: $\mathrm{y}=-107 \mathrm{x}+8.03, \mathrm{R}_{\text {adj }}^{2}=0.27, \mathrm{p}<1.10^{-100}$. 


\section{Field data and model parameterization}

\section{Characteristics of the dataset}

The Forest Inventory and Analysis (FIA) program of the US Forest Service comprises a cyclical inventory of forests in the conterminous 48 states of the US. Every year, one fifth of the FIA plots are revisited. For the analysis, we used the plots selected in ${ }^{11}$, which were plots east of the $100^{\text {th }}$ meridian that were fully forested, and not recently affected by human disturbance. In total, 207,444 plots in the FIA dataset met these criteria ${ }^{11}$. The $100^{\text {th }}$ meridian is historically seen as a separator of the humid eastern US and arid western US ecosystems ${ }^{66}$. Due to variation in sampling intensity within the FIA program, the focus on the eastern US also yielded a more contiguous dataset, with fairly consistent species pools throughout the sampling region ${ }^{11}$. Plot observations were aggregated at the level of 2 by 2 -degree grid cells (see also the subsection 'Selection of grid cell size' below). Within each plot (randomly located within a $24 \mathrm{~km}^{2}$ hexagon), four circular subplots are selected, each with a radius of $7.32 \mathrm{~m}$. In these subplots, trees with a diameter at breast height $(\mathrm{dbh})$ exceeding $12.7 \mathrm{~cm}$ are recorded. Previous analyses revealed that using either the number of trees or the sum of basal area as predictor variable yields similar patterns of frequency-dependence ${ }^{11}$. In the analyses presented here, we used the cumulative basal area in the subplot (in $\mathrm{m}^{2}$ ) as the predictor variable as it contains the more detailed information about the aboveground biomass. Hence, this predictor variable also provides a more detailed estimation than individual tree counts of the belowground biomass and influence of the target species on the local (soil) environment. Moreover, tree-size distributions can vary extensively across species, whereas basal area provides a way to standardize between trees of different age classes ${ }^{12,13}$. Within each subplot, a microplot with a radius of $2.07 \mathrm{~m}$ is established. Within each microplot, the species identity 
and abundance of seedlings smaller than $2.54 \mathrm{~cm}$ dbh and taller than $30.5 \mathrm{~cm}$ (hardwoods) or $12.24 \mathrm{~cm}$ (conifers) are recorded. The distance between subplots exceeds $30 \mathrm{~m}$, which means that subplots can be treated as independent sample units regarding the frequency-dependent effects of adult trees on the seedlings growing in the microplot within ${ }^{11}$. In the original analysis ${ }^{11}$, it was assumed that subplots could also be treated as independent sample units regarding the presence of seed sources (i.e. adult trees), but this assumption may exclude cases of seedling mortality in subplots where conspecific adults are absent ${ }^{32}$. Therefore, following Johnson et al. ${ }^{31}$, we considered here that seeds produced by adults may have been able to reach neighbouring subplots in which conspecific adults were absent. Moreover, when seedlings are present in neighbouring subplots, it is possible to rule out alternative factors suppressing seedling growth entirely for a species at the plot scale. Therefore, such observations at the plot scale were considered ecologically meaningful joint absences of seedlings and conspecific adults in this study, because the absence of seedlings may have been the result of seedling mortality, rather than a lack of a proximate seed source ${ }^{31}$ or alternative processes altogether suppressing a species' seedling growth. Hence, we analysed for each species $\mathrm{x}$ grid cell combination the set of subplots containing seedlings, conspecific and heterospecific adults ${ }^{11}$, as well as ecologically meaningful joint absences of seedlings and conspecific adults ${ }^{31}$. Figure S6 illustrates observations of seedling densities (in microplots), the mean seedling densities per microplot (the filled line in each panel of Fig. S6) and cumulative basal areas of adult trees (in subplots) species occurring more than 30 times within the particular grid cell (for the illustration we selected the grid cell covering $40-42{ }^{\circ} \mathrm{N}$, $\left.84-86^{\circ} \mathrm{W}\right)$. 

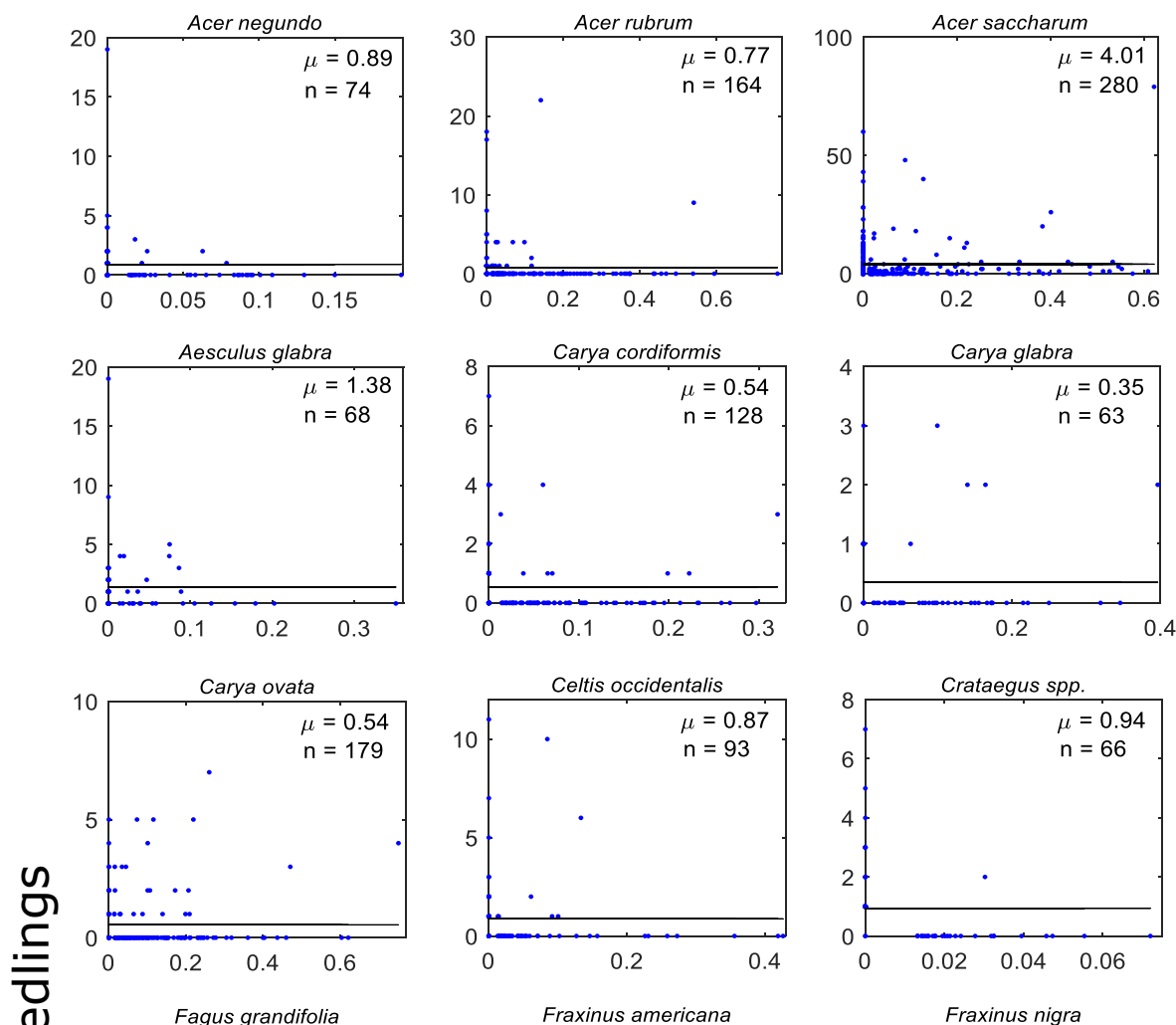

Supplementary Figure

S6 | Scatterplots

illustrating

observations of

seedling density (in

microplots) and adult

density (expressed in

cumulative basal area

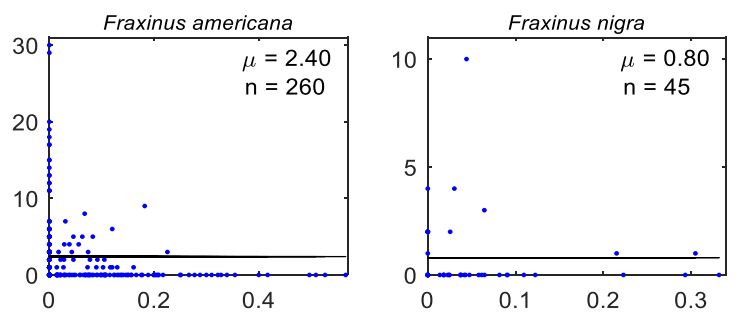

within associated

subplots) of one of the

2 by 2 degree grid

cells $\left(40-42{ }^{\circ} \mathrm{N}\right.$,

84-86 $\left.{ }^{\circ} \mathrm{W}\right)$. Each

100 Fraxinus pennsylvanica
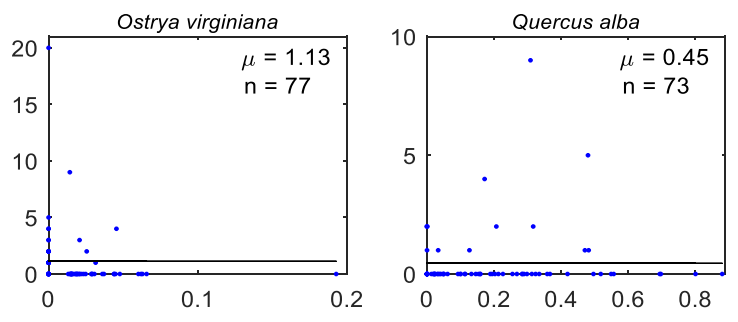

scatterplot indicates the

mean seedling number

per subplot $(\mu$, filled

black line) and the

number of subplots in

which the species was
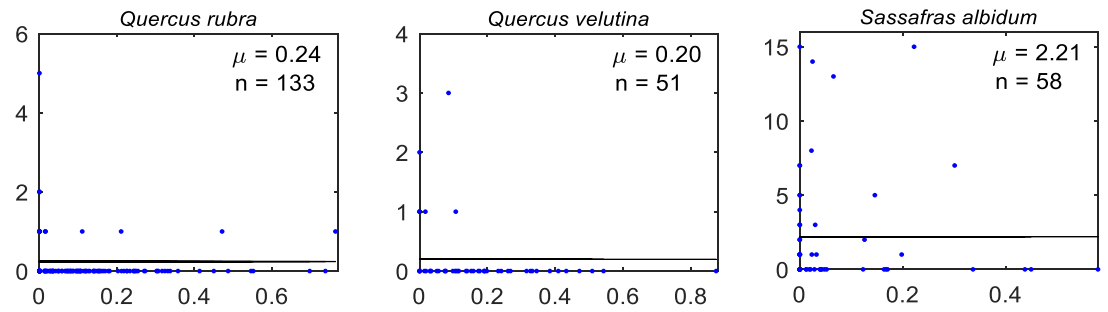

present as seedling and/or adult $(n)$.
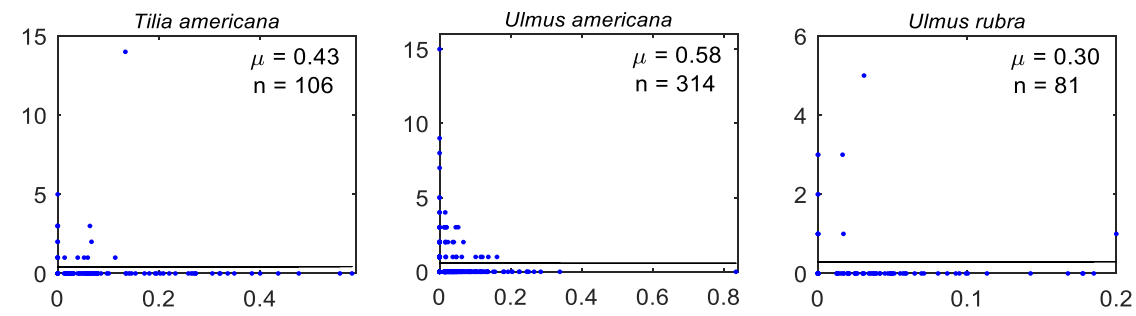

\section{Cumulative basal area $\left(\mathrm{m}^{2}\right)$}


In the analyses described above, adult density within the subplot is used as an indicator for the adult frequency experienced by seedlings. It is important to note that seedlings may in practice experience slightly different adult densities, due to the presence of large trees just outside the subplot, for example. These discrepancies, however, would most likely lead to underestimating adult densities when their densities are low within the subplot itself ${ }^{31}$. This implies that this effect may lead to estimations of frequency dependence that are somewhat conservative.

\section{$\underline{\text { Calculation of the coefficients }} \sigma_{i j}$ and model parameterization}

We used the dataset described above to parameterize the parameters quantifying species' growth rates in our theoretical model framework. Johnson et al. ${ }^{11,31}$ inferred the strength of density dependence from statistical relations between observed seedling densities in microplots and the tree density in the surrounding subplot. Given the apparent non-linear relationship in adult-seedling density scatterplots (e.g. Fig. S6), Johnson et al. ${ }^{11}$ considered an exponential function and a hyperbolic function to model this relationship. Both these functions include a single parameter quantifying the density-dependent effect on seedling abundance. Also, as it was clear that the (discrete) seedling count data was right-skewed and over dispersed (Fig. S6), negative binomial and Poisson distributions were considered in the candidate models. Combining AIC and examination of residuals for randomness, Johnson et al. ${ }^{11}$ selected the exponential function with a negative binomial error distribution as the fitting function. The model selection and fitting procedures were carried out in $\mathrm{R}$, using the mle 2 
function in the bbmle package ${ }^{11}$. Thus, the following function was fitted to the data to quantify conspecific effects:

$S_{i, i}\left(T_{i}\right)=S_{0_{i, i}} e^{b_{i, i} T_{i}}$

In similar vein, the following function was fitted to the data to quantify heterospecific effects:

$s_{i, j}\left(T_{j}\right)=s_{0_{i, j}} e^{b_{i, j} \sum_{i \neq j}^{n} T_{j}}$

Where $s_{i, j}$ is the expected number of seedlings of species $i$ in a microplot, based on the density-dependent effect exerted by adult conspecific $\left(T_{i}\right)$ or heterospecific $\left(T_{j}\right)$ trees. For brevity, we will use from here the notation $T_{k}$ to refer to either conspecific $\left(T_{k}=T_{i}\right)$ or heterospecific $\left(T_{k}=\sum_{j \neq i}^{n} T_{j}\right)$ adult trees. Further, $s_{0_{i, k}}$ is the expected number of seedlings of species $i$ in the absence of density-dependent effects exerted by species $k . T_{k}$ is the number of trees or the sum of the basal area $\left(\mathrm{L}^{2}\right)$ of species $k$ within the subplot, and $b_{i, k}$ is the parameter indicating the strength of density dependence of species $i$ on species $k\left(\mathrm{~L}^{-2}\right)^{18}$. Note that Johnson et al. ${ }^{11,31}$ specifically calculated conspecific frequency effects in this manner, i.e. $i=$ $k$, and that the average heterospecific effect was estimated by grouping all heterospecific adult trees. The fitting procedure yielded in a small number of cases of positive frequency dependence for particular species in specific grid cells. Figure S7 illustrates the results of the fitting procedure for the conspecific effects of community members of the same grid cell (40$\left.42{ }^{\circ} \mathrm{N}, 84-86^{\circ} \mathrm{W}\right)$ as before. The model fits in Figure S7 also illustrate the uncertainty in model fits, based on the standard error in the estimations of the parameters $b_{i, i}$. 

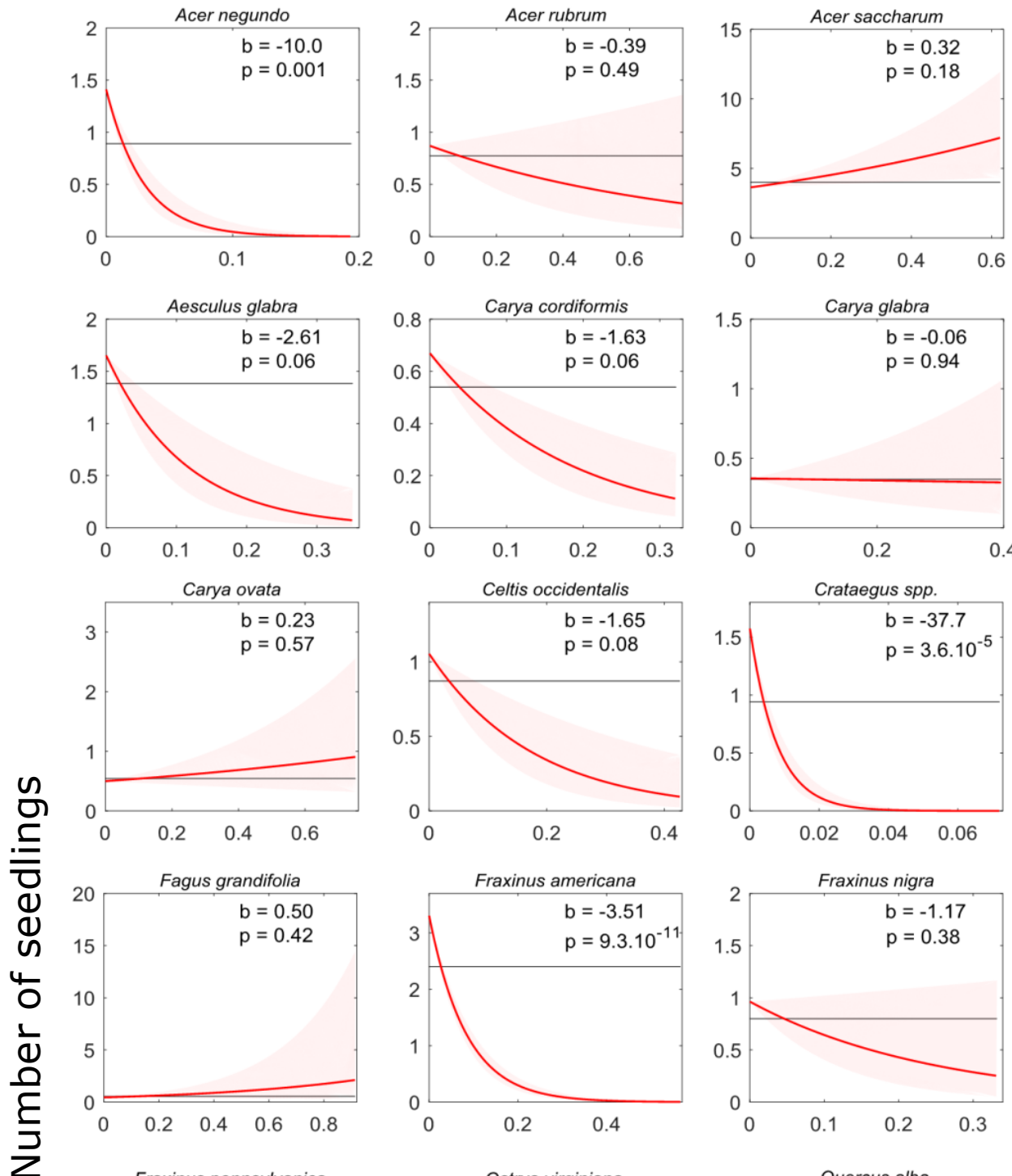

Supplementary

Figure S7 |

Scatterplots

illustrating the fits

of the function $a \mathrm{e}^{b T}$

(using maximum

likelihood and a

negative binomial

error

distribution ${ }^{11,31}$ ) to

the observations

shown in Figure S6.

Model fits are

indicated with red

lines. The lower
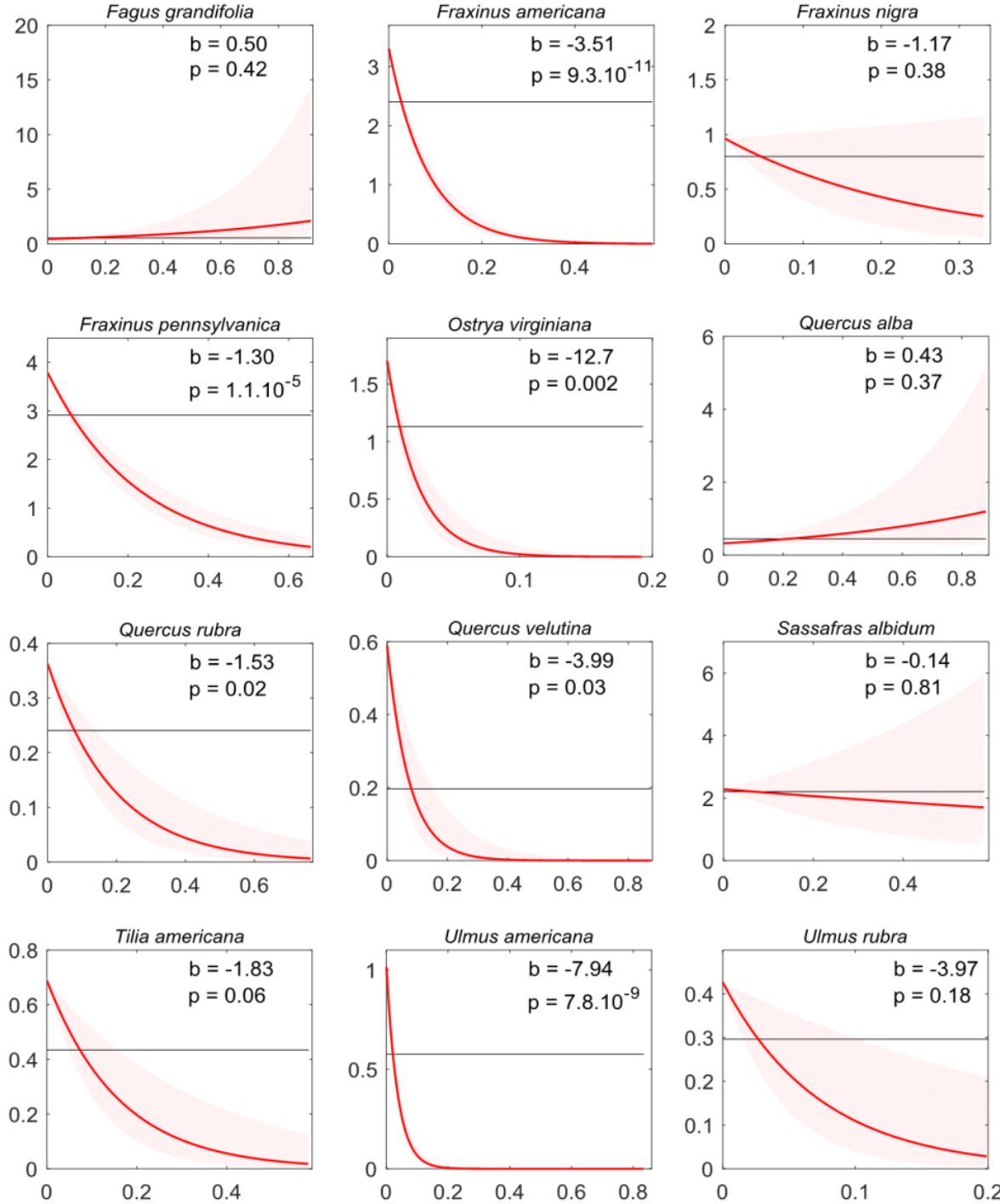

bound of the red

shaded area is

described by $a \mathrm{e}^{(b-\text {-s.e. }) T}$

and the upper

boundary is

described by

$a \mathrm{e}^{(\mathrm{b}+\text { s.e. }) T}$, where s.e.

is the standard error

for $b$ as determined

by the fitting

procedure. P-values

indicate the statistical

significance of the

negative exponential

curves that have been

fitted to the data. The

mean seedling

density (black line) is again plotted for comparison with Fig.

S6. 
In Figure S7, the y-axes have been centred around the mean seedling densities that were already shown in Figure S6. Given that mean and fitted seedling densities (Fig. S7) are typically much lower than the maximum observed densities (Fig. S6), it seems reasonable to assume that crowding effects on seedlings are minor within the range of the fitted functions for density dependence.

From an ecological perspective, the (negative) function shapes can be interpreted as the per capita seedling mortality rate increasing linearly as a function of (conspecific or heterospecific) adult density:

$\frac{d s_{i}}{d t}=\mu b_{i, k} s_{i} T_{k}$

In which $b_{i, k}$ is again the per (conspecific or heterospecific adult) capita change in number of seedlings due to density-dependent processes and $\mu$ is the characteristic response rate of seedlings to changes in adult density (dimension: per time). Solving equation [S68] analytically for the characteristic response time $t=\mu^{-1}$ (following ${ }^{67}$ ) yields equations [S67]. We note that in the approach described above, variation in seed rain and initial recruitment were not considered. It seems likely that with increasing adult density, both seed rain and hence initial recruitment would increase as well. Within the dataset used in this study, however, it is not possible to disentangle these positive effects of increasing adult density from the negative density-dependent effects of adults on seedling density. Therefore, we assume that seed rain and initial recruitment are constant (i.e. independent of adult density). 
This assumption likely leads to underestimation of negative density-dependent effects, making our analysis more conservative.

From equations [S67] we can derive the per capita relative rate of change in seedling density:

$\frac{1}{\mu^{-1}} \frac{s_{i, k}\left(T_{k}+1\right)-s_{i, k}\left(T_{k}+1\right)}{s_{i, k}\left(T_{k}\right)}=\mu \frac{s_{0_{i, k}} e^{b_{i, k}\left(T_{k}+1\right)}-s_{0_{i, k}} e^{b_{i, k} T_{k}}}{s_{0_{i, k}} e^{b_{i, k} T_{k}}}=\frac{\mu s_{0_{i, k}} e^{b_{i, k} T_{k}}\left(e^{b_{i, k}}-1\right)}{s_{0_{i, k}} e^{b_{i, k} T_{k}}}=\mu\left(e^{b_{i, k}}-1\right)$

From equation [S69] we can observe that the per capita relative rate of change in seedling density is constant.

In our current framework, plant species densities are modelled as frequencies varying between 0 and 1 (following ${ }^{18}$ ), rather than biomass densities or cumulative basal area with species-specific carrying capacities (as e.g. done in ${ }^{19,22,41}$ ). Including this assumption enables us to focus on relative differences, eliminating a priori differences in seedling density based on plant growth form and differences in life history strategies. We can therefore scale equation [S67] through division by a characteristic seedling density. Here, it is important to take into account that a small proportion of the statistical model fits yielded positive values for $b_{i, j}$ (as mentioned above; refs. $\left.{ }^{11,31}\right)$, Therefore, we needed to ensure in the scaling method used that positive and negative frequency-dependent effects were dealt with in a balanced (i.e. symmetrical) way ${ }^{68,69}$. Continuing from equation [S69], this was obtained through:

$\eta_{i, k}=\frac{1}{\mu^{-1}} \frac{s_{i, k}\left(T_{k}+1\right)-s_{i, k}\left(T_{k}\right)}{s_{i, k}\left(T_{k}+1\right)-s_{i, k}\left(T_{k}\right)}=\frac{1}{\mu^{-1}} \frac{\mu s_{0_{i, k}} e^{b_{i, k} T_{k}}\left(e^{b_{i, k}}-1\right)}{\mu s_{0_{i, k}} e^{b_{i, k} T_{k}}\left(e^{b_{i, k}}+1\right)}=\frac{1}{\mu^{-1}} \frac{e^{b_{i, k}}-1}{e^{b_{i, k}}+1}$

Where $\eta_{i, k}$ is the per (adult of species $k$ ) capita change in seedling growth of species $i$, which occurs over the characteristic response time. In plant-soil feedback experiments, the characteristic response time is the time needed for a species to completely shape the soil environment $^{18,21}$. It is reasonable to assume that in forest ecosystems, the characteristic 
response time is determined by the timespan between different cohorts of germinating seedlings (i.e. one year). Given that this response time is relatively short compared to the average age of adult trees with a $\mathrm{dbh} \geq 12.7 \mathrm{~cm}\left(\right.$ e.g. $>20-45$ years $\mathrm{in}^{70}$ ), current adult cumulative basal area provides an accurate estimate for the average adult density that was present over the characteristic response time.

When we assume that crowding mainly limits the density and size of adult trees, and that growth of a population is driven by seedlings developing to become adults, we can use the growth term in a single-species version of the density-dependent model (that was introduced in Supplementary Information section 1, equation [S4]), to calculate the per capita change in growth (due to conspecific effects) in this framework:

$\left.\frac{d N_{i}}{d t}\right|_{N_{i}+1}-\left.\frac{d N_{i}}{d t}\right|_{N_{i}}=\sigma_{i i}\left(N_{i}+1\right)-\sigma_{i i} N_{i}=\sigma_{i i}$

As the dimension of $\sigma_{i i}$ is per time, we can link the two frameworks by expressing the $\sigma_{i i}$ coefficients in the same units as the characteristic response time (i.e. per year). Independently of conspecific adult tree abundance, heterospecific effects quantified by $\sigma_{i j}$ can be derived by the same procedure ${ }^{11}$. As previously noted, the frequency-dependent feedback model used in this study (also introduced in Supplementary Information section 1, equation [S12]) is a generalization of the plant-soil feedback model developed by ${ }^{18}$, as described in ${ }^{33}$. One of the modifications is that the model parameters are rescaled to quantify negative frequency dependence as a value between 0 (very strong negative frequency dependence) and 1 (no negative frequency dependence). Application of this rescaling yields:

$\sigma_{i, k}=1+\eta_{i, k}=1+\frac{1}{\mu^{-1}}\left(\frac{e^{b_{i, k}}-1}{e^{b_{i, k}}+1}\right)$ 
A visual illustration of the approach is provided in Figure S8.

a

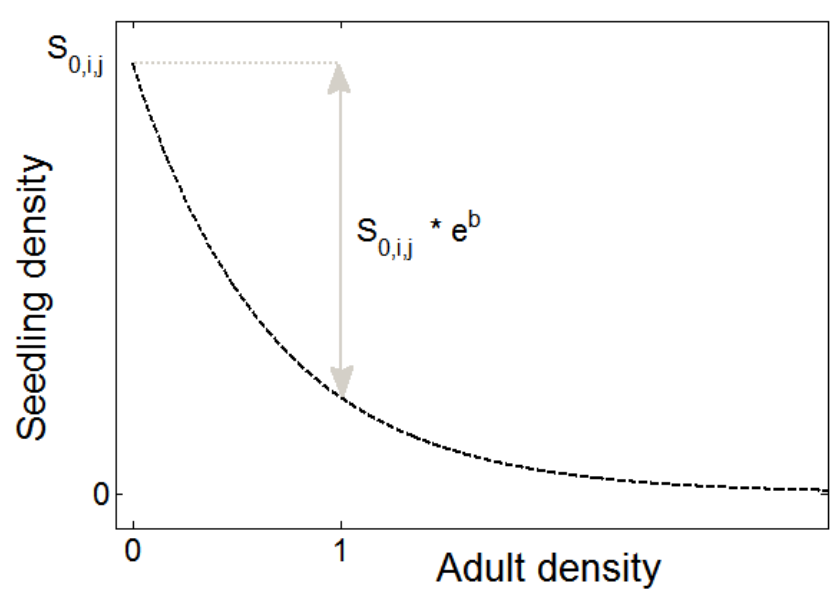

b

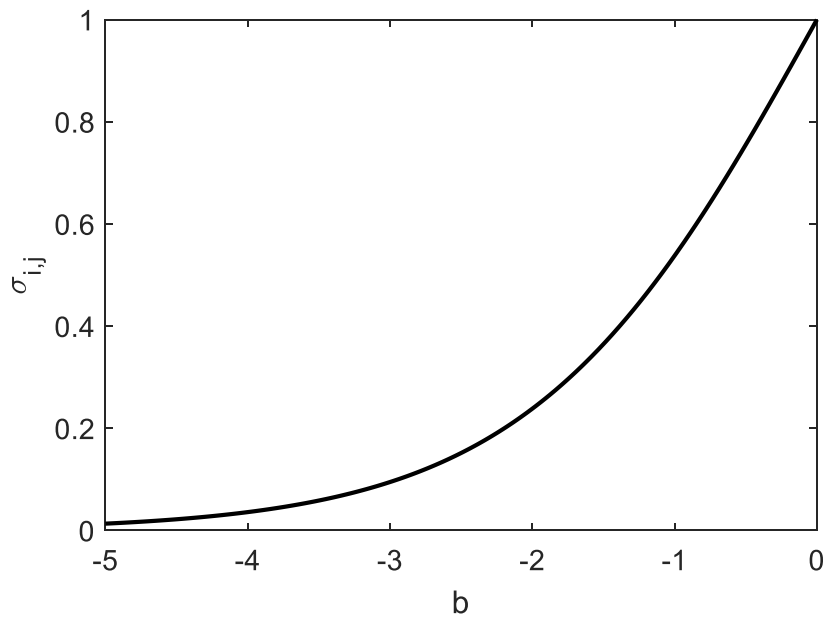

Supplementary Figure S8 | The relation between the coefficient of density dependence, $b$ (from ${ }^{11}$ ), and the parameters $\sigma_{i, j}$ of the frequency-dependent model framework utilized in this study. a) Similar to the plantsoil feedback approach of ${ }^{18}$, seedling density in 'trained' versus 'untrained' soils can be estimates as a function of $b$. b) A graph illustrating the relation (equation [S67] in the text) between $b$ (as results from fitting of field data using maximum likelihood ${ }^{11,31}$ ), and the parameters $\sigma_{i, j}$ of the frequency-dependent model utilized in this study.

In the parameterization procedure, we also took into account that a given model estimate for the parameter $b_{i, j}$ could be associated with varying degrees of error (e.g. Fig. S7). More specifically, we generated for each grid cell 1,000 dataset replicates. Within each replicate, we sampled for each species a $b_{i, j, r e p}$-value from the uniform distribution $\mathrm{U}\left(b_{i, j}-\right.$ s.e., $b_{i, j}+$ s.e.), in which s.e. indicates the standard error associated with the $b_{i, j}$-value obtained for that specific species $\mathrm{x}$ grid cell combination with the fitting procedure described above. Subsequently, these $b_{i, j, r e p}$-values were transformed to a model coefficient $\sigma_{i j, \text { rep }}$. The conspecific and heterospecific coefficients for each species were then calculated as the 
average value of the $1,000 \sigma_{i j, \text { rep }}$-values for that particular grid cell. Converting the seedling data $^{11,31}$ to model parameters in this manner reveals that in the dataset analysed, conspecific effects were much stronger than heterospecific effects (Fig. S9). Moreover, conspecific effects were more strongly negative in grid cells with higher community diversity (Fig. S9a; Pearson's $\mathrm{r}_{111}=-0.47, \mathrm{p}=2 \cdot 0 \cdot 10^{-7}$ ). A positive trend was found for heterospecific effects, in that heterospecific effects were less negative (i.e. closer to 1) in grid cells with higher community diversity (Fig. S9b; Pearson's $\mathrm{r}_{111}=0.29, \mathrm{p}=0.0016$ ).

The observed variation in coefficients obtained with the FIA dataset (Fig. S9) were used to generate random species interaction matrices, enabling the comparison between observed species richness patterns and simulated patterns driven entirely by variation in the strength of community-level feedback. As noted in Supplementary Information section 4, the (row-) averaged heterospecific effects in the dataset excluded the possibility of studying potential intransitive structures in the real communities, and future empirical studies would benefit from using full-factorial designs to assess all heterospecific effects occurring in the community (see also Supplementary Information section 7). To assess within-row variation, we assumed that variation in average heterospecific effects within a community were similar to variation within a species' experienced heterospecific effects (following ${ }^{23}$ ). 

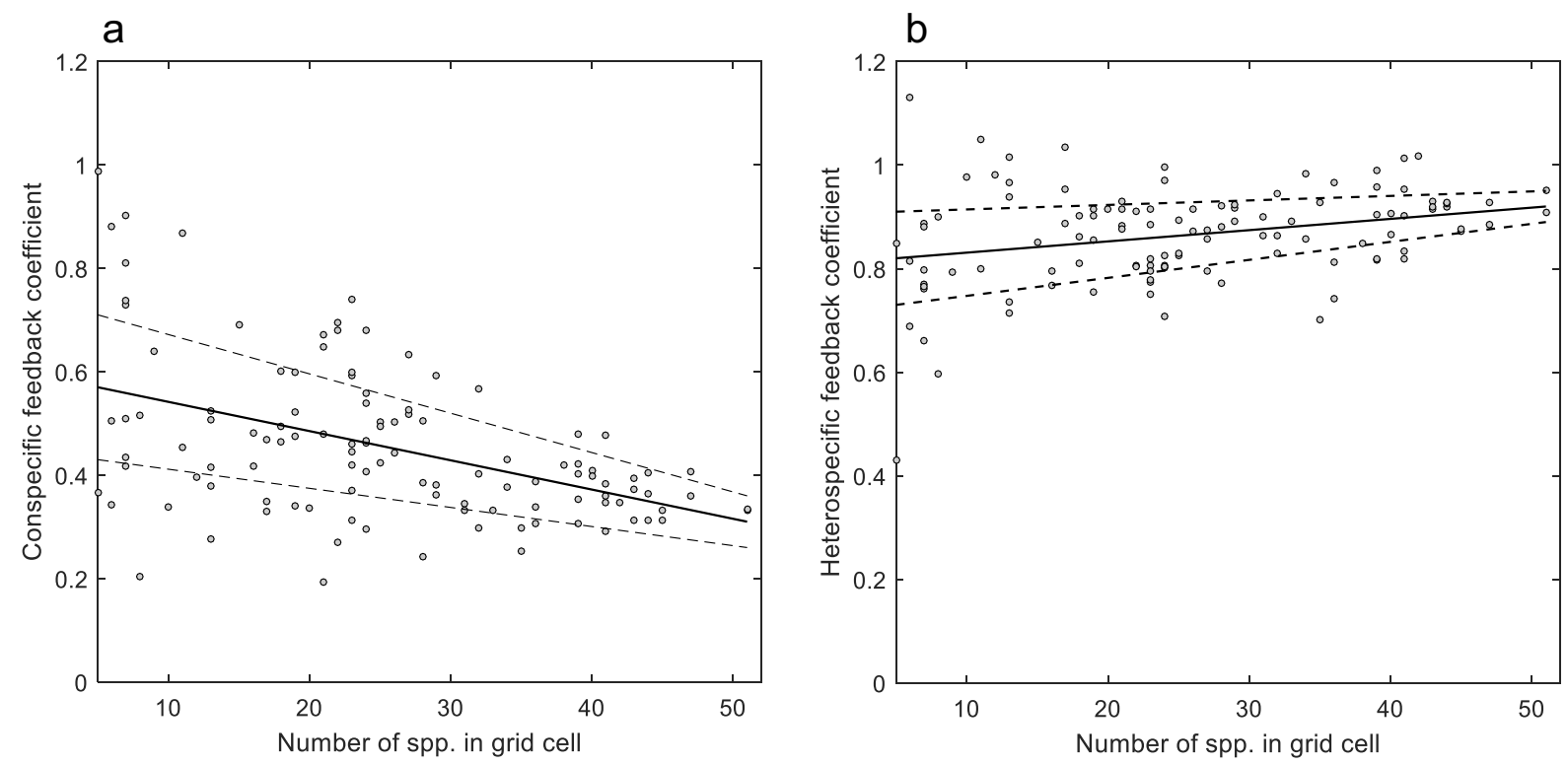

Supplementary Figure S9 | Average values of a) conspecific and b) heterospecific effects, for deciduous forest communities, aggregated into 113 grid cells, as a function of the number of species present in each grid cell. a) Feedback coefficients, $\sigma$, indicate whether effects are neutral $(\sigma=1)$, positive $(\sigma>1)$ or negative $\sigma<1)$. Conspecific effects $\sigma_{i, i}$ are more negative than heterospecific effects $\sigma_{i, j}$, and grid cells with higher species richness are characterized by more strongly negative conspecific effects (Pearson's $\mathrm{r}_{111}=-0.47, \mathrm{p}=$ 2.0.10 $0^{-7}$ ). A positive trend was found for heterospecific effects, in that heterospecific effects were less negative in grid cells with higher community diversity (Fig. S7B; Pearson's $r_{111}=0.29, p=0.0016$ ). Variation in the strength of community level feedback was based on these observations, meaning that more negative feedbacks were generated by stronger conspecific and slightly weaker heterospecific feedbacks (filled lines in both panels). In the simulations shown in Figure 4 of the main text, parameter values were drawn from a normal distribution around the means, the standard deviation used for conspecific and heterospecific coefficients is based on fitting the $68.2 \%$ confidence interval around the trend (the dotted lines in both panels $\mathbf{a}$ and $\mathbf{b}$ ).

To test whether our fitting procedure created a bias towards negative conspecific and heterospecific effects, we randomized within each grid cell the microplot seedling densities for each species, while keeping the adult densities in the associated subplots intact. Removing the association between microplots and subplots in this manner, we found that both 
conspecific and heterospecific effects were much weaker in the randomized dataset than in the real dataset (conspecific effects (Fig. S10a): $\sigma_{i, i, \text { data }}=0.46, \sigma_{i, i, \text { random }}=0.93, \mathrm{t}_{112}=27.8, \mathrm{p}=$ 4.7.10-55; heterospecific effects (Fig. S10b): $\sigma_{i, j, \text { data }}=0.86, \sigma_{i, j, \text { random }}=0.97, \mathrm{t}_{112}=12.5, \mathrm{p}=$ 6.2.10 ${ }^{-23}$ ). Moreover, in the randomized dataset there were no significant trends in the strength of conspecific effects (Fig. S10a; Pearson's $\mathrm{r}_{110}=-0.05, \mathrm{p}=0.58$ ) and heterospecific effects (Fig. S10b; Pearson's $\mathrm{r}_{110}=0.15, \mathrm{p}=0.12$ ) with increasing levels of community diversity. These results show that the trends in our dataset cannot be explained by an inherent bias resulting from the data selection and fitting procedure.
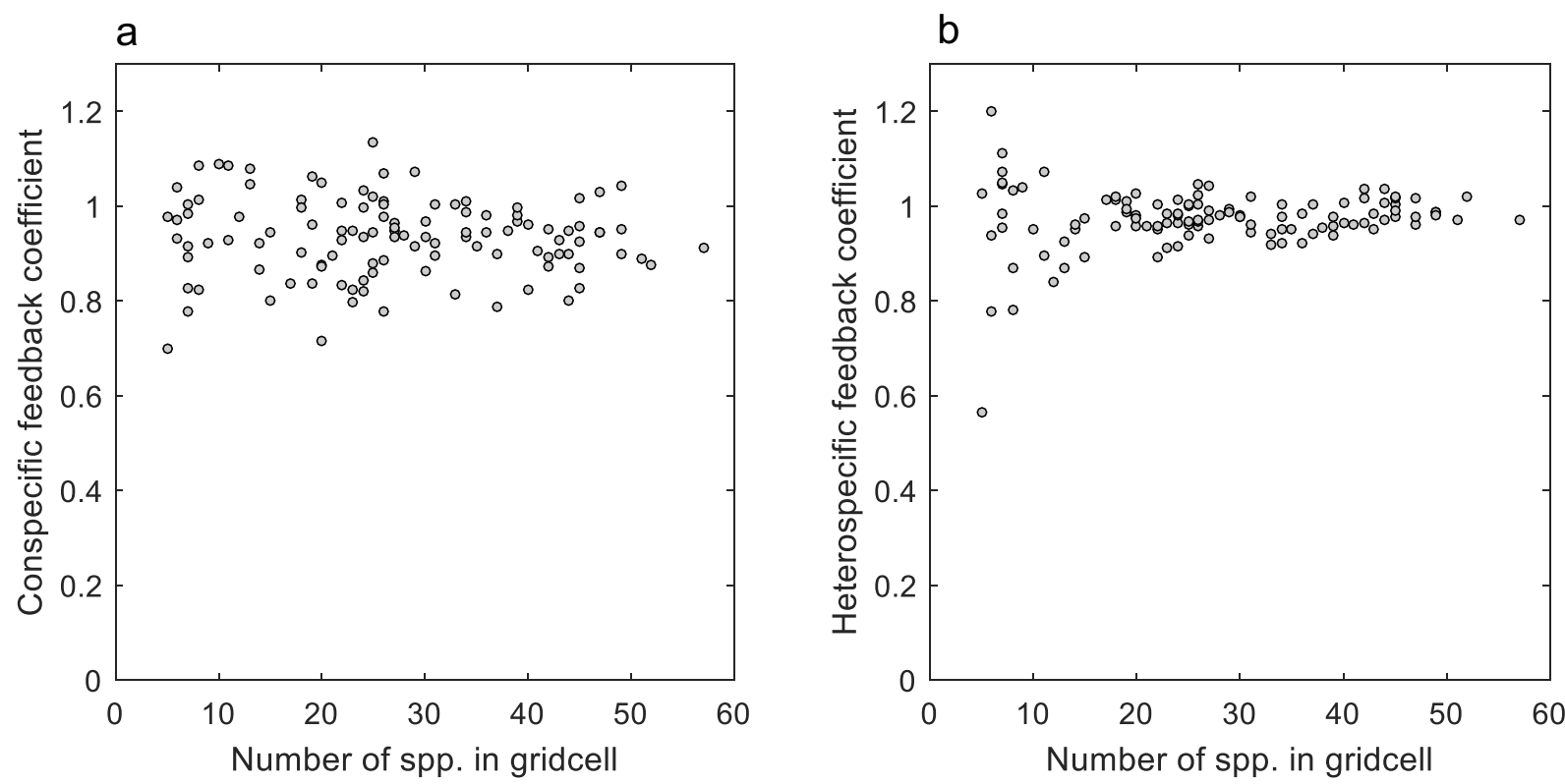

Figure S10 | Effects of randomization of seedling data on the calculated average values of a) conspecific effects and b) heterospecific effects. After randomization, conspecific and heterospecific effects are much weaker than observed in the real dataset (see also Figure S7 for comparison). Pair-wise t-tests were performed to compare calculated effects in the randomized and actual dataset (conspecific effects: $\sigma_{i, i, d a t a}=0.46$, $\sigma_{i, i, \text { random }}=0.93, \mathrm{t}_{12}=27.8, \mathrm{p}=4.7 .10^{-55}$; heterospecific effects: $\sigma_{i, j, \text { data }}=0.86, \sigma_{i, j, \text { random }}=0.97, \mathrm{t}_{111}=12.5$, $\mathrm{p}=6.2 \cdot 10^{-23}$ ). In addition, the randomized dataset did not exhibit a relationship between plant community diversity within a grid cell and the strengths of conspecific effects (correlation test: Pearson's $\mathrm{r}_{111}=-0.05$, $\mathrm{p}=0.58)$ and heterospecific effects (correlation test: Pearson's $\mathrm{r}_{110}=0.15, \mathrm{p}=0.12$ ). 


\section{$\underline{\text { Selection of grid cell size }}$}

It is well known that the direction and strength of ecological relations as inferred from aggregation of local site data may depend on the grain of aggregation (e.g. $\left.{ }^{71}\right)$. We examined the potential effect of grain size on data availability and capturing the community diversity gradient spanned by the eastern US deciduous forests. We note that some of these data could be excluded from subsequent analyses however, as the selected fitting function used may not converge for each species by cell combination ${ }^{11,31}$. As at least 30 observations (of either adult trees or seedlings) within a grid cell were used to fit functions describing conspecific and heterospecific density dependence ${ }^{11,31}$, a problem may emerge when the grain size of aggregation is small. More specifically, not enough data may be available even though a species' frequency of occurrence would be high enough to be included within an analysis using a larger grid size. In the dataset, this effect was clearly present when analysing community diversity patterns at 1 by 1 and 1.5 by 1.5 degrees, where the two most diverse species classes would be much more often represented if the necessary frequency of occurrence was about four times smaller ( $\mathrm{n}=8$, corresponding to an analysis in which the grid cell length is approximately doubled, Fig. S11a). As a result, the low diversity classes become over-represented at small grid cell sizes of aggregation. In contrast, observations of community diversity aggregated at larger grid cell sizes tended to be more robust against further increases in grid cell size (Fig. S11b). A problem with large grid cell sizes of aggregation, however, is that much of the variation in community diversity is lost, as the low diversity classes become underrepresented (Fig. S11a).

At the grain size of 2 by 2 degrees, the observed gradient in community diversity is relatively robust to increases in grid cell size (Fig. S11a). Also, the gradient observed comes closest to 
an equal representation of each species diversity categories that we selected, when compared to alternative grid cell sizes of aggregation (Fig. S11b).For slightly smaller grid cell sizes, the lack of evenness is compensated for by the total number of observations (i.e. grid cells) increasing (Fig. S11a) and therefore would not be particularly problematic for the statistical power of tests comparing differences between the groups. As noted above, the main problem at smaller grid cell sizes is that many species do not meet the criterion of $n=30$ observations, even though their frequency of occurrence would be sufficient to be included in larger grid cells. Hence, given the constraints of our analysis, a grid cell size of 2 by 2 degrees is coming closest to optimizing the amount of information that can be extracted for our analysis (Fig. S11). 

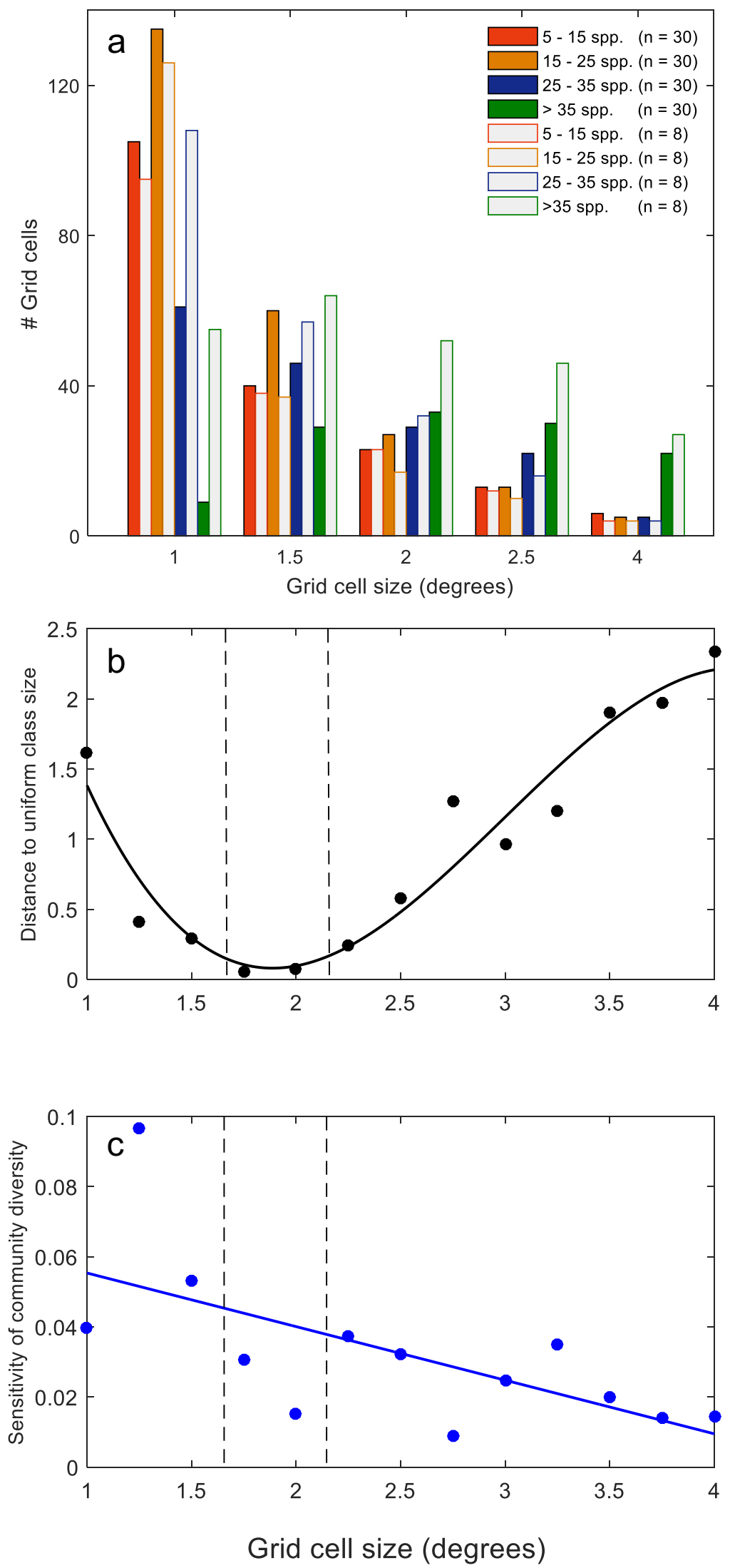


\section{Supplementary Figure S11 | Analysis of how patterns in community diversity depend on the spatial grain}

(i.e. grid cell) size of aggregation. a) Due to the requirement of 30 observations per species per grid cell to fit the density dependent relation $s_{i, j}\left(T_{j}\right)=s_{0_{i, j}} e^{b_{i, j} T_{j}}$, an increasing number of species fails to meet this requirement with decreasing grid cell size. This leads to an underrepresentation of the high diversity classes. In contrast, large grid cells are relatively robust to further increases in size, but smoothen out gradients in community diversity, leading to an overrepresentation of the high diversity classes. b) The above pattern can be quantified by means of the distance to uniform class size, which is calculated here as: $\sum_{\mathrm{i}=1}^{4}\left(\frac{4 \mathrm{p}_{\mathrm{i}}}{\mathrm{n}}-1\right)^{2}$, where $n$ is the total number of grid cells, and $p_{i}$ the number of grid cells falling within community diversity category $i$. At intermediate grid sizes, a relatively even distribution of among the diversity classes is observed, while being relatively robust to changes in grid cell size. Given the criteria set for this study (number of observations per grid cell, and the ranges of the community diversity classes), grid cells of sizes around $2 * 2$ degrees optimize the information that can be extracted from the FIA dataset. Filled line shows a cubic fit: $y=-0.39 x^{3}+3.5 x^{2}-$ $\left.9.1 \mathrm{x}+7.4, \mathrm{R}_{\mathrm{adj}}{ }^{2}=0.90, \mathrm{p}=0.006 \mathrm{c}\right)$ The sensitivity of community diversity to increases in grid cell size can also be quantified, showing the decreasing sensitivity with increasing grid cell size. Here, the specific metric is calculated as: $\sum_{\mathrm{i}=1}^{4}\left(\mathrm{p}_{\mathrm{i}}-\mathrm{q}_{\mathrm{i}}\right)^{2}$, where $p_{i}$ the number of grid cells falling within community diversity category $\mathrm{i}$ when the standard number of observations for species inclusion is used $(\mathrm{n}=30)$. Further, $q_{i}$ the number of grid cells falling within community diversity category $i$ when the number of observations for species inclusion is based on the density needed to be included when the length of the grid cell would be (approximately) doubled $(n=8)$. As we cannot estimate losses of data due to a lack of a converging fitting solution, we compared the total number of species by cell combinations for each grid size and observation number. Filled line shows a linear fit: $\left.y=-0.015 x+0.07, \operatorname{Radj}^{2}=0.36, p=0.018\right)$. 


\section{Robustness of the plant-soil feedback - species diversity relation}

In Figure 2 of the main text, the relation between the strength of feedback and community diversity was established through systematic variation of model parameters. More specifically, a feedback gradient was generated through sampling along an axis of variation in conspecific coefficients and heterospecific coefficients, which varied in opposite directions along this axis (Fig. S12a). We examined the robustness of the obtained feedback-diversity relation by using alternative modes of parameter variation as well. More specifically, we examined cases in which there was no correlation (Fig. S12d) or a positive correlation (Fig. $\mathrm{S} 12 \mathrm{~g}$ ) between conspecific and heterospecific coefficients. Regardless of the correlation structure between conspecific and heterospecific coefficients, we found that more diverse communities persisted under more strongly negative community-level feedback (Fig. S12b, $\mathrm{S} 12 \mathrm{e}, \mathrm{S} 12 \mathrm{~h})$, and that average pair-wise feedback was an accurate indicator of communitylevel feedback (Fig. S12c, S12f, S12i). These results indicate that the findings presented in the main text are not specific to the parameter generation algorithm for the theoretical communities assembled. 

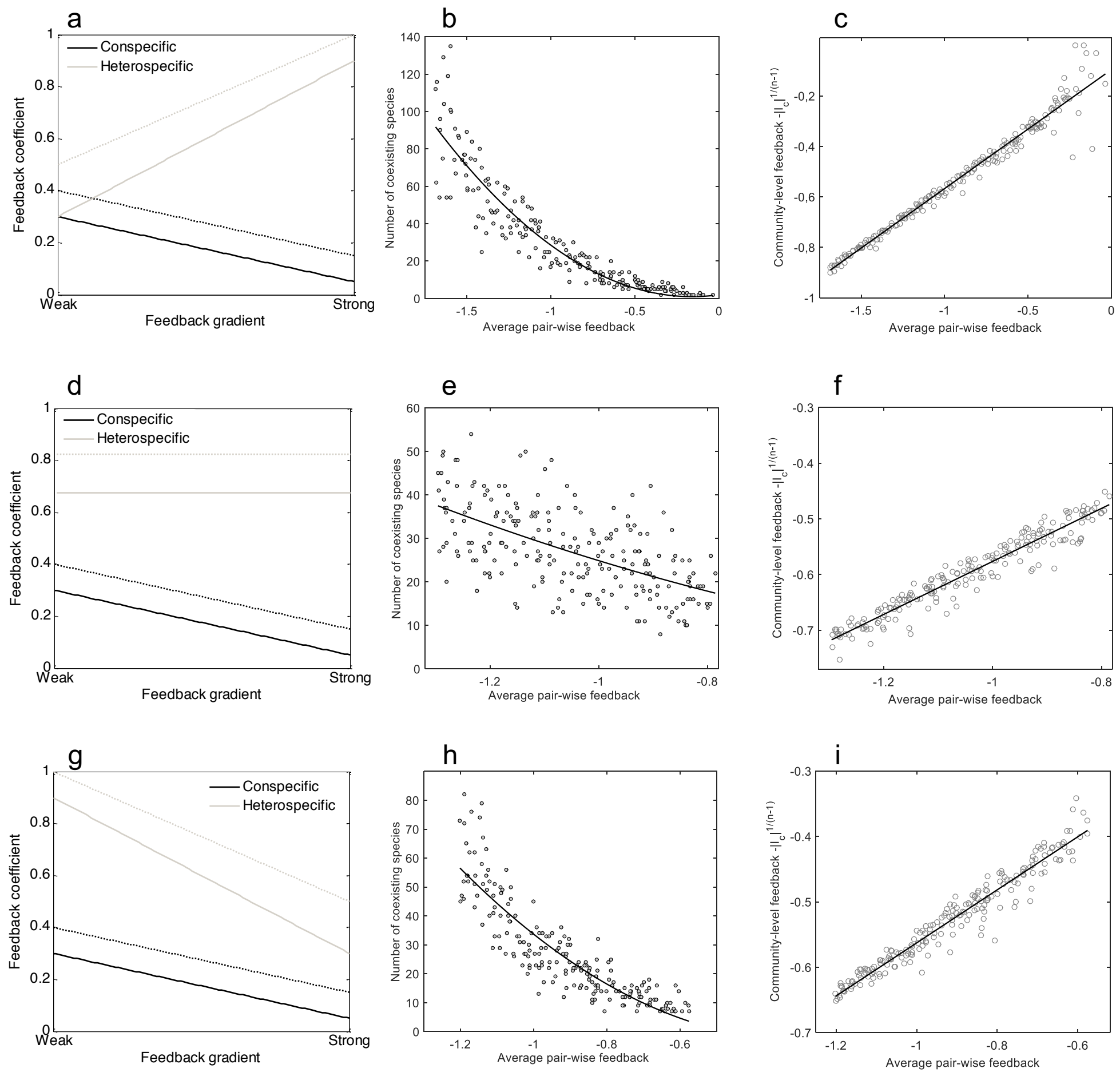

Supplementary Figure S12 | Examining the relation between strength of negative frequency-dependent feedback and diversity using different algorithms of parameter variation. Feedback strengths were varied using positive, negative and no correlation between conspecific and heterospecific effects. For all three regimes, the number of coexisting species increased under more strongly negative feedback, and under all regimes average pair-wise feedback was an accurate indicator of community-level feedback. a) Negative correlation 
between conspecific and heterospecific coefficients. For each position along the feedback gradient, conspecific and heterospecific coefficients are drawn from a uniform distribution ranging from the filled lines (minimum values) to the dashed lines (maximum values). b) The feedback - diversity relation under negative correlation. Filled line shows a quadratic fit assuming a minimum community size of 2 plant species $\left(y=39.00 x^{2}+12.52 x\right.$, $\left.\mathrm{R}_{\mathrm{adj}}{ }^{2}=0.86\right)$. c) The relation between average pair-wise feedback and community-level feedback under negative correlation. Filled line shows a linear fit $\left(\mathrm{y}=0.47 \mathrm{x}-0.10, \mathrm{R}_{\mathrm{adj}}{ }^{2}=0.97\right)$. d) No correlation assumed between conspecific and heterospecific coefficients. The meaning of the filled and dashed lines is the same as in a) e) The feedback - diversity relation without correlation. Filled line shows a quadratic fit assuming a minimum community size of 2 plant species $\left(y=15.23 \mathrm{x}^{2}-7.66 \mathrm{x}, \mathrm{R}_{\mathrm{adj}}{ }^{2}=0.35\right)$. f) The relation between average pair-wise feedback and community-level feedback without correlation. Filled line shows a linear fit $(\mathrm{y}=0.48 \mathrm{x}-0.11$, $\mathrm{R}_{\mathrm{adj}}{ }^{2}=0.93$ ). g) Positive correlation between conspecific and heterospecific coefficients. The meaning of the filled and dashed lines is the same as in a) h) The feedback - diversity relation under positive correlation. Filled line shows a quadratic fit assuming a minimum community size of 2 plant species $\left(y=68.11 x^{2}+36.42 x\right.$, $\left.\mathrm{R}_{\mathrm{adj}}{ }^{2}=0.79\right)$. i) The relation between average pair-wise feedback and community-level feedback under positive correlation. Filled line shows a linear fit $\left(\mathrm{y}=0.41 \mathrm{x}-0.16, \mathrm{R}_{\mathrm{adj}}{ }^{2}=0.94\right)$.

The above results, together with the results presented in Supplementary Information section 4, suggest that the strength of correlation between community-level feedback and averagepairwise feedback may depend on the specific parameterization of conspecific and heterospecific conspecific coefficients. Because we could only infer average pair-wise feedbacks strengths from our empirical data from eastern US deciduous forest (Supplementary Information section 5), it is relevant to verify that for the parameterization obtained (Fig. S9), a strong correlation between community-level feedback, $I_{C}$, and average pair-wise feedback is likely. Calculations of $I_{C}$ for the plant communities shown in Fig. 4 of the main text, confirmed that the correlation between community-level feedback and average pair-wise feedback is strong under the specific parameter constraints derived (Fig. S13). 


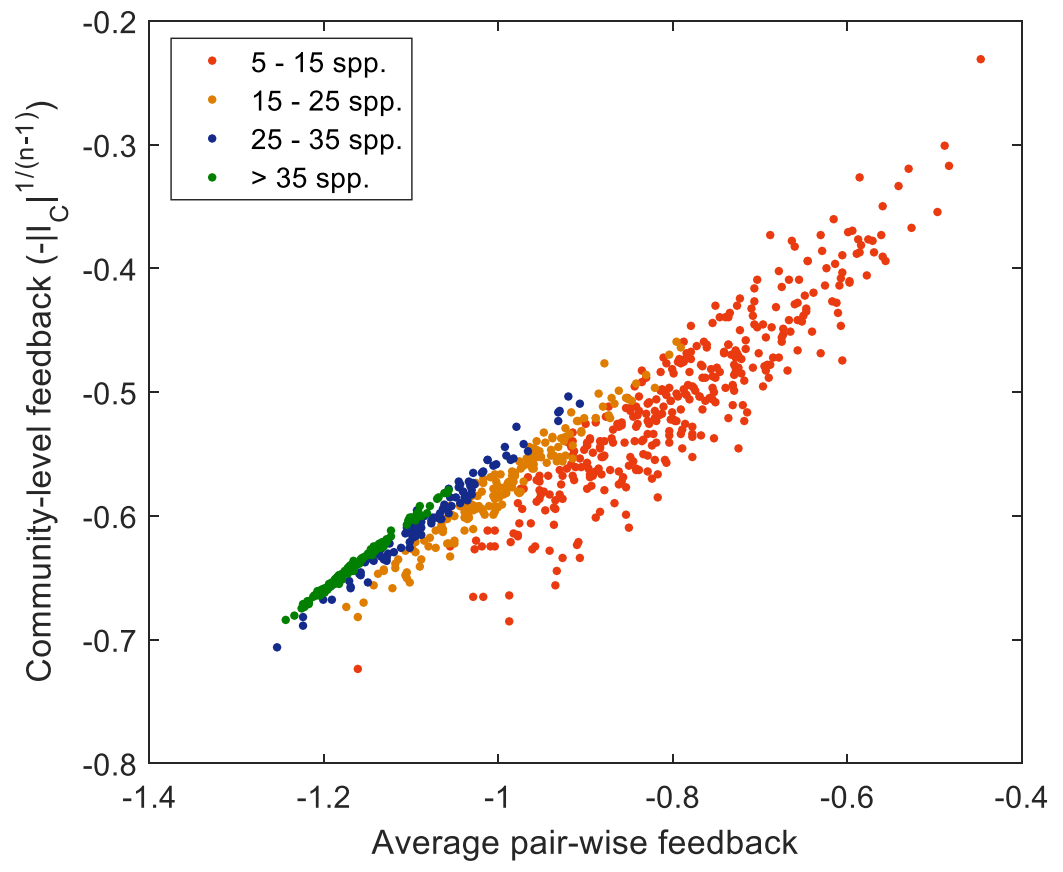

Supplementary Figure S13 | Examining the strength of the correlation between average pair-wise feedback and community-level feedback, $I_{C}$, when conspecific and heterospecific coefficients are constrained by the empirical data from deciduous forest communities: These results suggest that the variation in inferred heterospecific effects is relatively small compared to the variation in inferred conspecific effects, explaining why average pair-wise feedback provides an accurate indication of community-level feedback when the model parameters have been constrained according to the field data (Pearson correlation tests: $5-15$ species: $\mathrm{r}_{366}=0.925, \mathrm{p}=1.9 .10^{-155} ; 15-25$ species: $\mathrm{r}_{175}=0.976, \mathrm{p}=6.2 .10^{-117} ; 25-35$ species: $\mathrm{r}_{101}=0.988, \mathrm{p}=3.9 .10^{-85} ;>35$ species: $\left.\mathrm{r}_{127}=0.997, \mathrm{p}=1.2 .10^{-142}\right)$. 


\section{Relaxing assumptions of zero-sum dynamics and fitness equality}

In this section, we show that the approach to calculate coexistence equilibria (Supplementary Information section 3), also applies to the model system describing plant population sizes, rather than frequencies (the latter involving a zero-sum assumption). Subsequently, we show that inherent fitness inequalities (e.g. ${ }^{6,42}$ ) can be introduced in the framework relatively easily. Finally, we show that inclusion of these inherent fitness inequalities affects the feasibility of the equilibrium, but not community-level feedback $I_{C}$.

\section{Equilibrium solution when relaxing the zero-sum assumption}

In Supplementary Information section 3, the equilibrium solution for the replicator equation was described, using the zero-sum assumption (i.e. $\sum_{i=1}^{n} P_{i}=1$, meaning $\sum_{i=1}^{n} \frac{d P_{i}}{d t}=0$ at any time $t$ ). Here we show that the same approach can be used to solve the model system describing plant population sizes (equation [S5]), meaning that the zero-sum assumption has been relaxed. We can generalize equation [S5] to find a solution for an $n$ species system:

$$
\frac{d N_{i}}{d t}=\left(\frac{\sum_{j=1}^{n} \sigma_{i j} N_{j}}{\sum_{j=1}^{n} N_{j}}-\rho \frac{\sum_{j=1}^{n} N_{j}}{K}\right) N_{i}
$$

To find the equilibrium solution, we set the right-hand side of equation [S73] to zero, and using matrix notation, we obtain: 
A. $\widehat{\boldsymbol{N}}=\left[\begin{array}{cccc}\sigma_{11} & \sigma_{12} & . . & \sigma_{1 n} \\ \sigma_{21} & . . & . . & . . \\ . . & . . & . . & . . \\ \sigma_{n 1} & . . & . . & \sigma_{n n}\end{array}\right] \cdot\left[\begin{array}{c}\hat{N}_{1} \\ \hat{N}_{2} \\ . . \\ \hat{N}_{n}\end{array}\right]=\frac{\rho}{K}\left(\sum_{j=1}^{n} N_{j}\right)^{2} . \mathbf{1}$

In which 1 specifies a column vector of length $n$. And, because $\rho, K>0$, we can solve equation [S74] using Cramer's rule to obtain:

$\frac{\hat{N}_{i}}{\left(\sum_{j=1}^{n} N_{j}\right)^{2}}=\frac{\rho}{K} \frac{\operatorname{det} \mathbf{A}_{i}}{\operatorname{det} \mathbf{A}}$

Now, we can use equation [S75] to write:

$\sum_{i=1}^{n}\left(\frac{\hat{N}_{i}}{\left(\sum_{j=1}^{n} \hat{N}_{j}\right)^{2}}\right)=\frac{1}{\sum_{j=1}^{n} \hat{N}_{j}}=\frac{\rho}{K \operatorname{det} \mathbf{A}} \sum_{k=1}^{n} \operatorname{det} \mathbf{A}_{k}$

Substituting equation [S76] in equation [S75] and rearranging then yields:

$$
\hat{N}_{i}=\frac{K \operatorname{det} \mathbf{A} \operatorname{det} \mathbf{A}_{i}}{\rho\left(\sum_{j=1}^{n} \operatorname{det} \mathbf{A}_{j}\right)^{2}}=\frac{K \operatorname{det} \mathbf{A}}{\rho \sum_{j=1}^{n} \operatorname{det} \mathbf{A}_{j}} \frac{\operatorname{det} \mathbf{A}_{i}}{\sum_{j=1}^{n} \operatorname{det} \mathbf{A}_{j}}
$$

Note that the second factor in equation [S77] is identical to the full solution for the system describing plant frequencies (equation [4] in the main text). As we considered a system with equivalent carrying capacities $K$ and the same response rate to crowding $\rho$ for all species, the first factor is a constant (given a certain interaction matrix $\mathbf{A}$ ) and plant frequencies can thus be converted to plant numbers. The normalization constant involved in this conversion 
will change if there is a change in the structure of the interaction matrix A. Equation [S77] also shows that solutions of the model systems analysed do not crucially depend on the zerosum assumption.

\section{$\underline{\text { Introducing inherent fitness inequalities }}$}

Following ${ }^{42,72}$, plant growth can be decomposed into a frequency-dependent component and a frequency-independent component. The latter component then describes inherent fitness inequalities (as defined by ${ }^{6,42}$ ) between plant species. Introducing such a term in equation [S5], we can write:

$\frac{d N_{i}}{d t}=\left(W_{i}-f(\vec{N})+r_{i}\right) N_{i}=\left(\frac{\sum_{j=1}^{n} \sigma_{i j} N_{j}}{\sum_{j=1}^{n} N_{j}}-f(\vec{N})+r_{i}\right) N_{1}$

In which $r_{i}$ is the frequency-independent per capita growth rate of species $i\left(\mathrm{~T}^{-1}\right)$. Because equation [S78] can be rewritten as:

$\frac{d N_{i}}{d t}=\left(\frac{\sum_{j=1}^{n} \sigma_{i j} N_{j}}{\sum_{j=1}^{n} N_{j}}+\frac{\sum_{j=1}^{n} r_{i} N_{j}}{\sum_{j=1}^{n} N_{j}}-f(\vec{N})\right) N_{1}=\left(\frac{\sum_{j=1}^{n}\left(\sigma_{i j}+r_{i}\right) N_{j}}{\sum_{j=1}^{n} N_{j}}-f(\vec{N})\right) N_{1}$

We see that the model structure remains largely intact after the inclusion of inherent fitness inequalities. More specifically, using the same choice for $f(\vec{N})$ as in equation [S5], and applying the chain rule (as described in equations [S7] - [S9]), it follows that the system's feedbacks are now described by: 
$w_{i}=\sum_{j=1}^{n}\left(\sigma_{i j}+r_{i}\right) P_{j}$

As a result, the analytical framework (presented in the main text and analysed in more detail in Supplementary Information section 3) would still apply to this extended model, the only difference being that elements in the interaction matrix $\mathbf{A}^{\prime}$ now contain a frequencydependent and a frequency-independent term:

$$
\mathbf{A}^{\prime}=\left[\begin{array}{cccc}
\sigma_{11}+r_{1} & \sigma_{12}+r_{1} & . . & \sigma_{1 n}+r_{1} \\
\sigma_{21}+r_{2} & . . & . . & . . \\
. . & . . & . . & . . \\
\sigma_{n 1}+r_{n} & . . & . . & \sigma_{n n}+r_{n}
\end{array}\right]
$$

In cases where full-factorial experiments enable the parameterization of all coefficients $\sigma_{i j}$, discrepancies between calculated (using equation [4] in the main text) and observed equilibrium frequencies could provide indications of the importance of inherent fitness inequalities between species. More specifically, species with $r_{i}-\bar{r}>0$ will have higher frequencies when these inherent fitness inequalities are included. As a result, inherent fitness inequalities could change whether the system's feasibility condition for coexistence is fulfilled or not. We will now show, however, that these inherent fitness inequalities do not change the community-level feedback $I_{C}$. Using equations [S50] and [S81] we can write:

$$
I_{C}=(-1)^{n} \sum_{i=1}^{n} \operatorname{det} \mathbf{A}_{i}^{\prime}
$$

From equations [S81] and [S82] it becomes clear that additional terms emerge in the calculations of the determinants of $\mathbf{A}_{i}^{\prime}$ as compared to the determinants of $\mathbf{A}_{i}$ (which 
determine $I_{C}$ in the model without inherent fitness inequalities). We will now proceed with calculating the difference between these two models' $I_{C}$ :

$$
\Delta I_{C}=(-1)^{n} \sum_{i=1}^{n} \operatorname{det} \mathbf{A}_{i}^{\prime}-\operatorname{det} \mathbf{A}_{i}
$$

And show that this difference is null in all cases. First, we can use the Laplace expansion (as was done in Supplementary Information section 3) to write these additional terms emerging from the determinants of $\mathbf{A}_{i}^{\prime}$ in the following structure:

$\operatorname{det} \mathbf{A}_{i}^{\prime}-\operatorname{det} \mathbf{A}_{i}=\sum_{j=1}^{n}(-1)^{j+1} r_{j} \sum_{k \neq i}^{n}(-1)^{k+1} \operatorname{det} \check{\breve{A}}_{i \mid j k}$

Where det $\breve{\mathbf{A}}_{i \mid j k}$ indicates the determinant of a square matrix of $n$-1 rows and columns, which is obtained by removing the $j^{\text {th }}$ row and the $k^{\text {th }}$ column from matrix $\mathbf{A}_{i}$ (i.e. matrix $\mathbf{A}$ with the $i^{\text {th }}$ column substituted with a column vector of ones). As the derivation of equation [S84] is somewhat lengthy, we will leave this derivation for the end of this section. Now, we can sum equation [S83] for all $n$ species in the community by writing:

$$
\left|\Delta I_{C}\right| \propto \sum_{i=1}^{n} \operatorname{det} \mathbf{A}_{i}^{\prime}-\sum_{i=1}^{n} \operatorname{det} \mathbf{A}_{i}=\sum_{i=1}^{n} \sum_{j=1}^{n}(-1)^{j+1} r_{j} \sum_{k \neq i}^{n}(-1)^{k+1} \operatorname{det} \breve{\mathbf{A}}_{i \mid j k}
$$

From equation [S85], we can now observe that for a fixed value of $j$, the expansion yields determinants of an always even number of $n(n-1)$ matrices. This number emerges through the exclusion of the set of $n$ matrices for which $i=k=x$, as $\operatorname{det} \breve{\mathbf{A}}_{x \mid j x}=0$ (which is shown in the derivation at the end of this section). Importantly, each of these $n(n-1)$ matrices $\breve{\mathbf{A}}_{p \mid j q}$ can be 
paired to a matrix $\breve{\mathbf{A}}_{q \mid j p}$, which is composed of the same elements from the matrix A. More specifically, in both matrices $\breve{\mathbf{A}}_{p \mid j q}$ and $\breve{̆}_{q \mid j p}$ the $j^{\text {th }}$ row, as well as the $p^{\text {th }}$ and $q^{\text {th }}$ columns have been removed. The two matrices, however, are different regarding the position of the column that is equal to 1 . Therefore, their determinants can be related by exchanging columns $p$ - $q$-1 times if $p>q$, or $q-p$ - 1 times if $p<q$. These relationships can be written with the same cofactor as:

$\operatorname{det} \breve{\mathbf{A}}_{p \mid j q}=(-1)^{p-q-1} \operatorname{det} \breve{\mathbf{A}}_{q \mid j p}$

Subsequently, we can add the other co-factors for each of the two matrices by substituting $p$ and $q$ for $i$ and $k$ in equation [S85]. Setting $i=p$ and $k=q$ then yields:

$r_{j}(-1)^{q+j} \operatorname{det} \breve{\mathbf{A}}_{p \mid j q}=r_{j}(-1)^{q+j+p-q-1} \operatorname{det} \breve{\mathbf{A}}_{q \mid j p}=r_{j}(-1)^{j+p-1} \operatorname{det} \breve{\mathbf{A}}_{q \mid j p}$

In addition, we obtain the co-factor for the paired matrix by setting $i=q$ and $k=p$ :

$r_{j}(-1)^{p+j} \operatorname{det} \breve{\mathbf{A}}_{q \mid j p}$

It is then clear that equations [S87] and [S88] cancel out. Hence, we have shown that all the determinants summing up to $\Delta I_{C}$ are either zero or cancel out, which effectively proves that $\Delta I_{C}$ is equal to zero.

This result thus shows an important property of community-level feedback $I_{C}$. We showed before that $I_{C}<0$ is a necessary condition for stable community coexistence. Inherent fitness inequalities are difficult to quantify ${ }^{42}$, but the above proof reveals that there is no way in which inherent fitness inequalities can stabilize communities if $I_{C}>0$. Only in cases where $I_{C}$ 
$<0$, but a coexistence equilibrium is not feasible (because of negative frequencies of the weakest species at equilibrium), inherent fitness inequalities may enable stable coexistence. Interestingly, from a more theoretical perspective, this result highlights the usefulness of $I_{C}$ as a metric to assess community stability. From studies of the replicator equation it is well known that the feasibility of the equilibrium is not affected when the matrix $\mathbf{A}$ is changed through multiplication or addition of a constant, or when a different constant is added to each column of the interaction matrix ${ }^{14}$. Although the equilibrium point, its eigenvalues and its eigenvectors do change when a different constant is added to each row of $\mathbf{A}$, it is useful to know that community-level feedback does not, and that addition of different constants to each row will not generate coexistence in a system where $I_{C}>0$. Also, these results suggest that our conclusions regarding community-level feedback and species coexistence do not critically depend on the assumption of inherent fitness equality as assumed when using the replicator equation to describe plant community dynamics. For example, (as shown in Fig. 1 of the main text), a shift from $I_{C}<0$ to $I_{C}>0$ can only be achieved by changes in frequencydependent interactions within a community, and this loss of stability of the community coexistence equilibrium could not be undone by any set of changes in species' inherent fitness differences.

These results suggest a potential way forward to studying frequency-dependent interactions in diverse plant communities. Full-factorial experimental designs (pot or field) involving all the community's species enables the quantification of all coefficients $\sigma_{i j}$. In turn, this enables the calculation of $I_{C}$, and an assessment of whether the community-level feedback within the species group is negative, and thus whether coexistence is possible. In case of $I_{C}<0$, parameters $r_{j}$, could then be used to fit model densities to observed densities, which would 
provide an estimate of the relative importance of frequency-independent (i.e. equalizing ${ }^{6}$ ) and frequency-dependent (stabilizing ${ }^{6}$ ) mechanisms to community structure. Such a fully parameterized model could then be used to study the effect of species removal, resistance to invasion by new species, and resilience to disturbances. A fully parameterized interaction matrix $\mathbf{A}$ is an important first step. Although it may be advantageous to group all heterospecific treatments to reduce the size of experiments, such designs do not render sufficient information to follow the approach outlined above. The provision of the theoretical tools needed to analyse the outcome of full-factorial community experiments might be a further motivation to undertake these experiments, and the generation of full-factorial datasets.

\section{Derivation of equation [S84]}

In order to calculate the consequences of frequency-independent growth terms for the value of $I_{C}$ we first examine what additional terms arise when calculating determinants. For notational convenience, we first consider:

$\operatorname{det} \mathbf{A}^{\prime}-\operatorname{det} \mathbf{A}=\operatorname{det}\left[\begin{array}{cccc}\sigma_{11}+r_{1} & \sigma_{12}+r_{1} & . . & \sigma_{1 n}+r_{1} \\ \sigma_{21}+r_{2} & . . & . . & . . \\ . . & . . & . . & . . \\ \sigma_{n 1}+r_{n} & . . & . . & \sigma_{n n}+r_{n}\end{array}\right]-\operatorname{det}\left[\begin{array}{cccc}\sigma_{11} & \sigma_{12} & . . & \sigma_{1 n} \\ \sigma_{21} & . . & . . & . . \\ . . & . . & . . & . . \\ \sigma_{n 1} & . . & . . & \sigma_{n n}\end{array}\right][\mathrm{S} 89]$

Where we can expand $\operatorname{det} \mathbf{A}^{\prime}$ as follows:

$$
\operatorname{det} \mathbf{A}^{\prime}=\operatorname{det}\left[\begin{array}{cccc}
\sigma_{11} & \sigma_{12} & . . & \sigma_{1 n} \\
\sigma_{21}+r_{2} & . . & . . & . . \\
. . & . . & . . & . . \\
\sigma_{n 1}+r_{n} & . . & . . & \sigma_{n n}+r_{n}
\end{array}\right]+\operatorname{det}\left[\begin{array}{cccc}
r_{1} & r_{1} & . . & r_{1} \\
\sigma_{21}+r_{2} & . . & . . & . . \\
. . & . . & . . & . . \\
\sigma_{n 1}+r_{n} & . . & . . & \sigma_{n n}+r_{n}
\end{array}\right]
$$


Now, using the same expansion on the second term on the right hand side of equation [S90] yields:

$$
\operatorname{det}\left[\begin{array}{cccc}
r_{1} & r_{1} & . . & r_{1} \\
\sigma_{21} & . . & . . & . . \\
. . & . . & . . & . . \\
\sigma_{n 1}+r_{n} & . . & . . & \sigma_{n n}+r_{n}
\end{array}\right]+\operatorname{det}\left[\begin{array}{cccc}
r_{1} & r_{1} & . . & r_{1} \\
r_{2} & r_{2} & . . & r_{2} \\
. . & . . & . . & . . \\
\sigma_{n 1}+r_{n} & . . & . . & \sigma_{n n}+r_{n}
\end{array}\right]
$$

The second term on the right hand side in equation [S91] equals zero, as the first two rows are multiples of each other. It is clear that the first matrix in equation [S91] can be expanded this way $n$-1 times (yielding $n-1$ determinants of zero, following the structure of the second matrix in equation [S91]), until the only non-zero determinant of this expansion remains:

$$
\operatorname{det}\left[\begin{array}{cccc}
r_{1} & r_{1} & . . & r_{1} \\
\sigma_{21} & . . & . . & . . \\
. . & . . & . . & . . \\
\sigma_{n 1} & . . & . . & \sigma_{n n}
\end{array}\right]=\operatorname{det} \mathbf{A}^{r_{1}}
$$

From here on we refer to this type of matrices as $\mathbf{A}^{r_{m}}$. Now, one can go back to the first matrix on the right hand side of equation [S91], and perform a similar expansion, yielding two matrices: 1) a matrix to be further expanded; 2) a matrix that has a non-zero determinant, in which all elements on the $2^{\text {nd }}$ row are given by $r_{2}$, and all other matrix elements are as in $\mathbf{A}$. Iteratively repeating the expansion with all the remaining matrices will thus yield a summation of $n$ determinants. Each of these determinants is from a matrix in which a single row contains elements $r_{j}$, and all other rows have the elements of A. Also, the final determinant left after all the expansions is $\operatorname{det} \mathbf{A}$ itself. Hence, we can write:

$$
\operatorname{det} \mathbf{A}^{\prime}-\operatorname{det} \mathbf{A}=\sum_{m=1}^{n} \operatorname{det} \mathbf{A}^{r_{m}}
$$


In which $\mathbf{A}^{r_{m}}$ indicates the matrix $\mathbf{A}$, with the $m^{\text {th }}$ row being replaced by a row vector of length $n$, with all elements equal to $r_{m}$. Analogous equality can be proven for matrices $\mathbf{A}_{i}$ and $\mathbf{A}_{i}$, noticing that all the matrices expanded as in equation [S91] will now have one column of zeros, and thus a zero determinant. The matrices analogous to the second term in equation [S90] will contain a zero instead of $r_{i}$ in the $i^{\text {th }}$-column, thus they also have a zero determinant. This is so because two rows are still multiples of each other if they share a zero value in the same column. Hence, it holds that:

$$
\operatorname{det} \mathbf{A}_{i}^{\prime}-\operatorname{det} \mathbf{A}_{i}=\sum_{m=1}^{n} \operatorname{det} \mathbf{A}_{i, m 0}^{r_{m}}
$$

In which $\operatorname{det} \mathbf{A}_{i, m 0}^{r_{m}}$ indicates the matrix $\mathbf{A}^{r_{m}}$ but with the $i^{\text {th }}$ column being replaced by a column vector (of length $n$ ) whose elements are ones, except for the element on row $m$ that is equal to zero. We now rewrite [S94] by means of a Laplace expansion of $\operatorname{det} \mathbf{A}_{i, m 0}^{r_{m}}$ along row $m:$

$$
\operatorname{det} \mathbf{A}_{i}^{\prime}-\operatorname{det} \mathbf{A}_{i}=\sum_{m=1}^{n} r_{m} \sum_{j \neq i}^{n}(-1)^{m+j} \operatorname{det} \breve{\mathbf{A}}_{i \mid m j}
$$

Which is equivalent to equation [S84]. Note that we only need to sum over $j \neq i$ as the $i^{\text {th }}$ element of row $m$ of matrix $\operatorname{det} \mathbf{A}_{i, m 0}^{r_{m}}$ is zero. The matrix $\breve{\mathbf{A}}_{i \mid m j}$ is obtained from $\mathbf{A}_{i}$ by removing the $m^{\text {th }}$ row and the $j^{\text {th }}$ column. 


\section{References}

33. Mack, K.M.L., Eppinga, M.B. \& Bever, J.D. Keystone competitors stabilize plant communities structured by frequency-dependent feedbacks. PLoS One, under review.

34. Hunt, R. \& Cornelissen, J.H.C. Components of relative growth rate and their interrelations in 59 temperate plant species. New Phyt. 135, 395-417 (1997).

35. Reinhart, K.O., Packer, A., Van der Putten, W.H. \& Clay, K. Plant-soil biota interactions and spatial distribution of black cherry in its native and invasive ranges. Ecol. Lett. 6, 1046-1050 (2003).

36. Van der Putten, W.H. et al. Empirical and theoretical challenges in abovegroundbelowground ecology. Oecologia 161, 1-14 (2009).

37. Van der Putten, W.H. et al. Plant-soil feedbacks: the past, the present and future challenges. J. Ecol. 101, 265-276 (2013).

38. Bever, J.D. Feedbacks between plants and their soil communities in an old field community. Ecology 75, 1965-1977 (1994).

39. Bever, J.D. et al. Rooting theories of plant community ecology in microbial interactions. Trends Ecol. Evol. 25, 468-478 (2010).

40. Levine, J.M. \& HilleRisLambers, J. The importance of niches for the maintenance of species diversity. Nature 461, 254-257 (2009).

41. Eppinga, M.B., Rietkerk, M., Dekker, S.C., De Ruiter, P.C. \& Van der Putten, W.H. Accumulation of local pathogens: a new hypothesis to explain exotic plant invasion. Oikos 114, 168-176 (2006).

42. Eppstein, M.J. \& Molofsky, J. Invasiveness in plant communities with feedbacks. Ecol. Lett. 10, 253-263 (2007).

43. Hofbauer, J. On the occurrence of limit cycles in the Lotka-Volterra equation. Nonlinear Anal. Theory Methods Appl. 5, 1003-1007 (1981).

44. Hofbauer, J. \& Sigmund, K. Evolutionary game dynamics. Bull. Amer. Math. Soc. 40, 479-519 (2003).

45. Cheung, Y.W. \& Friedman, D. A comparison of learning and replicator dynamics using experimental data. J. Econ. Behav. Organ. 35, 263-280 (1998).

46. Bomze, I.M. Lotka-Volterra equation and replicator dynamics: new issues in classification. Biol. Cybern., 72, 447-453 (1995).

47. Dambacher, J.M., Li, H.W. \& Rossignol, P.A. Relevance of community structure in assessing indeterminacy of ecological predictions. Ecology 83, 1372-1385 (2002).

48. Levins, R. Evolution in changing environments: some theoretical explorations (Princeton University Press, Princeton, NJ, USA, 1968).

49. Goh, B.S. Global stability in many-species systems. Am. Nat., 111, 135-143 (1977).

50. Berlow, E.L. et al. Interaction strengths in food webs: issues and opportunities. $J$. Anim. Ecol. 73, 585-598 (2004).

51. Kokkoris, G.D., Jansen, V.A.A., Loreau, M. \& Troumbis, A.Y. Variability in interaction strength and implications for biodiversity. J. Anim. Ecol. 71, 362-371 (2002).

52. Sandholm, W.H. Local stability under evolutionary game dynamics. Theor. Econ. 5, 27-50 (2010).

53. May, R.M. Qualitative stability in model ecosystems. Ecology 54, 638-641 (1973).

54. May, R.M. \& Leonard, W.J. Nonlinear aspects of competition between three species. SIAM J. Appl. Math. 29, 243-253 (1974). 
55. Olff, H., Hoorens, B., De Goede, R.M, Van der Putten, W.H. \& Gleichman, J.M. Small-scale shifting mosaics of two dominant grassland species: the possible role of soil-borne pathogens. Oecologia 125, 45-54 (2000).

56. Bever, J.D. Negative feedback within a mutualism: host-specific growth of mycorrhizal fungi reduces plant benefit. Proc. Roy. Soc. Lond. B 269, 2595-2601 (2002).

57. Stinson, K.A. et al. Invasive plant suppresses the growth of native tree seedlings by disrupting belowground mutualisms. PLoS Biol. 4, e140 (2006).

58. Bauer, J.T., Mack, K.M.L. \& Bever, J.D. 2015. Plant-soil feedbacks as drivers of succession: evidence from remnant and restored tallgrass prairies. Ecosphere 6, 1-12 (2015).

59. Vandermeer, J.H. Generalized models of two species interactions: a graphical analysis. Ecology 54, 809-818 (1973).

60. Laird, R.A. \& Schamp, B.S. Competitive intransitivity promotes species coexistence. Am. Nat. 168, 182-193 (2006).

61. De Ruiter, P.C., Neutel, A.M. \& Moore, J.C. Energetics, patterns of interaction strengths and stability in real ecosystems. Science 269, 1257-1260 (1995).

62. Schuster, P. Structure and dynamics of replication-mutation systems. Phys. Scr., 35, 402 (1987).

63. Schnabl, W., Stadler, P.F., Forst, C. \& Schuster, P. Full characterization of a strange attractor: chaotic dynamics in low-dimensional replicator systems. Physica $D, 48,65-$ 90 (1991).

64. Ohtsuki, H. \& Nowak, M.A. The replicator equation on graphs. J. Theor. Biol., 243, 86-97 (2006).

65.Hirsch, M.W., Smale, S. \& Devaney, R.L. Differential equations, dynamical systems, and an introduction to chaos ( $3^{\text {rd }}$ ed., Academic Press, Walton, MA, USA, 2012).

66. Powell, J.W. Report on the lands of the arid region of the United States, with a more detailed account of the lands of Utah $\left(2^{\text {nd }}\right.$ edition, Government Printing Office, Washington DC, 1879).

67. Bellows, T.S. The descriptive properties of some models for density dependence. $J$. Anim. Ecol. 50, 139-156 (1981).

68. Armas, C., Ordiales, R. \& Pugnaire, F.L. Measuring plant interactions: a new comparative index. Ecology 85, 2682-2685 (2004).

69. Diaz-Sierra, R., Verwijmeren, M., Rietkerk, M., Resco de Dios, V. \& Baudena, M. A new family of standardized and symmetric índices for measuring the intensity and importance of plant neighbor effects. Methods Ecol. Evol. 8, 580-591 (2017).

70. Lorimer, C.G. Age structure and disturbance history of a southern Appalachian virgin forest. Ecology 61, 1169-1184 (1980).

71. Baudena, M. et al. Revealing patterns of local species richness along environmental gradients with a novel network tool. Scientific Reports 5, 11561 (2015).

72. Eppinga, M.B., Pucko, C.A., Baudena, M., Beckage, B. \& Molofsky, J. 2013. A new method to infer vegetation boundary movement from 'snapshot' data. Ecography 36, 622-635 (2013). 